\title{
Blow up for the critical gKdV equation III: exotic regimes
}

\author{
YVAn MARTEl, Frank MERle ANd PIERRE RAPHAËL
}

\begin{abstract}
We consider the blow-up problem in $H^{1}$ for the $L^{2}$ critical generalized Korteweg-de Vries (gKdV) equation, as a continuation of $[38,39]$. We know from [38] that the unique and stable blow-up rate for $H^{1}$ solutions close to the solitons with strong decay on the right is
\end{abstract}

$$
\left\|u_{x}(t)\right\|_{L^{2}} \sim \frac{1}{T-t} \quad \text { as } t \uparrow T<+\infty .
$$

In this paper we construct non-generic blow-up regimes in $H^{1}$ by considering initial data with explicit slow decay on the right in space. We obtain finite time blow-up solutions with speed

$$
\left\|u_{x}(t)\right\|_{L^{2}} \sim \frac{1}{(T-t)^{v}} \quad \text { as } t \uparrow T<+\infty, \quad v>\frac{11}{13},
$$

as well as global in time growing up solutions with exponential growth

$$
\left\|u_{x}(t)\right\|_{L^{2}} \sim e^{t} \quad \text { as } t \rightarrow+\infty,
$$

or growth of any power

$$
\left\|u_{x}(t)\right\|_{L^{2}} \sim t^{\nu} \quad \text { as } t \rightarrow+\infty, \quad v>0 .
$$

These solutions can be taken with initial data arbitrarily close in $H^{1}$ to the ground state solitary wave.

Mathematics Subject Classification (2010): 35Q53 (primary); 35Q51, 35B44, 35B35 (secondary).

\section{Introduction}

\subsection{Setting of the problem}

We consider the $L^{2}$-critical generalized Korteweg-de Vries equation (gKdV)

$$
(\mathrm{gKdV}) \begin{cases}u_{t}+\left(u_{x x}+u^{5}\right)_{x}=0, & (t, x) \in[0, T) \times \mathbb{R} \\ u(0, x)=u_{0}(x), & x \in \mathbb{R} .\end{cases}
$$

P.R. is supported by the French ERC/ANR project SWAP. Part of this work was completed while P.R. was visiting the Mathematics Department at MIT which he would like to thank for its kind hospitality. This work is also supported by the project ERC 291214 BLOWDISOL.

Received September 12, 2012; accepted April 23, 2013. 
The Cauchy problem is locally well-posed in the energy space $H^{1}$ from Kenig, Ponce and Vega [20,21]. Given $u_{0} \in H^{1}$, there exists a unique ${ }^{1}$ maximal solution $u(t)$ of (1.1) in $C\left([0, T), H^{1}\right)$ with either $T=+\infty$, or $T<+\infty$ and then $\lim _{t \rightarrow T}\left\|u_{x}(t)\right\|_{L^{2}}=+\infty$.

For $H^{1}$ solution, the mass and the energy are conserved by the flow: $\forall t \in$ $[0, T)$,

$$
M(u(t))=\int u^{2}(t)=M\left(u_{0}\right), \quad E(u(t))=\frac{1}{2} \int u_{x}^{2}(t)-\frac{1}{6} \int u^{6}(t)=E\left(u_{0}\right) .
$$

Equation (1.1) has the following invariances: if $u(t, x)$ is solution of (1.1) then $-u(t, x), u(-t,-x)$ and

$$
\lambda_{0}^{\frac{1}{2}} u\left(\lambda_{0}^{3}\left(t-t_{0}\right), \lambda_{0}\left(x-x_{0}\right)\right),\left(\lambda_{0}, t_{0}, x_{0}\right) \in \mathbb{R}_{+}^{*} \times \mathbb{R} \times \mathbb{R}
$$

are also solutions of (1.1).

The family of traveling wave solutions of (1.1), called solitons, plays a distinguished role in the analysis:

$$
u(t, x)=Q_{\lambda_{0}}\left(x-\lambda_{0}^{-2} t-x_{0}\right), \quad\left(\lambda_{0}, x_{0}\right) \in \mathbb{R}_{+}^{*} \times \mathbb{R},
$$

with

$$
Q_{\lambda}(x)=\frac{1}{\lambda^{\frac{1}{2}}} Q\left(\frac{x}{\lambda}\right), \quad Q(x)=\left(\frac{3}{\cosh ^{2}(2 x)}\right)^{\frac{1}{4}}, \quad Q^{\prime \prime}+Q^{5}=Q .
$$

It is well-known that the function $Q$ is related to the following sharp GagliardoNirenberg inequality [65]:

$$
\forall v \in H^{1}, \quad \int|v|^{6} \leq \int v_{x}^{2}\left(\frac{\int v^{2}}{\int Q^{2}}\right)^{2} .
$$

Moreover, from (1.3), mass and energy conservations, for initial data in $H^{1}$ such that $\left\|u_{0}\right\|_{L^{2}}<\|Q\|_{L^{2}}$, the corresponding solution $u(t)$ of (1.1) is bounded in $H^{1}$ and thus globally defined in time.

\subsection{On the classification of the flow near $Q$}

For

$$
\|Q\|_{L^{2}}<\left\|u_{0}\right\|_{L^{2}}<\|Q\|_{L^{2}}+\alpha_{0}, \quad \alpha_{0} \ll 1
$$

the blow-up problem has been first studied in a series of works by Martel and Merle [31-34,44]. In particular, from a rigidity theorem around solitons [31], the first proof of blow-up in finite or infinite time was obtained [44] for initial data

$$
u_{0} \in H^{1} \text { such that }(1.4) \text { and } E\left(u_{0}\right)<0 .
$$

${ }^{1}$ In a certain sense. 
Recently, in $[38,39]$, the authors of the present paper have revisited the blow-up analysis for data near the ground state. First, in the so-called minimal mass case $\left\|u_{0}\right\|_{L^{2}}=\|Q\|_{L^{2}}$, the following existence and uniqueness results complement results in [35].

Minimal mass blow-up solution [39, 35]. (i) Existence. There exists a solution $S(t) \in \mathcal{C}\left((0,+\infty), H^{1}\right)$ to $(1.1)$ with minimal mass $\|S(t)\|_{L^{2}}=\|Q\|_{L^{2}}$ such that

$$
\left\|S_{x}(t)\right\|_{L^{2}} \sim \frac{\left\|Q^{\prime}\right\|_{L^{2}}}{t} \text { as } t \downarrow 0 .
$$

(ii) Uniqueness. Let $u$ be an $H^{1}$ blow-up solution of (1.1) with minimal mass $\|u(t)\|_{L^{2}}=\|Q\|_{L^{2}}$. Then $u=S$ up to the invariances of the $(\mathrm{gKdV})$ equation.

Second, [38,39] yield a classification of the flow for initial data close to $Q$ with decay on the right in space. More precisely, let

$$
\begin{gathered}
\mathcal{A}=\left\{u_{0}=Q+\varepsilon_{0} \text { with }\left\|\varepsilon_{0}\right\|_{H^{1}}<\alpha_{0} \text { and } \int_{y>0} y^{10} \varepsilon_{0}^{2}<1\right\}, \\
\mathcal{T}_{\alpha^{*}}=\left\{u \in H^{1} \text { with } \inf _{\lambda_{0}>0, x_{0} \in \mathbb{R}}\left\|u-Q_{\lambda_{0}}\left(.-x_{0}\right)\right\|_{L^{2}}<\alpha^{*}\right\} .
\end{gathered}
$$

Then the following classification result holds:

Classification in $\mathcal{A}\left[\mathbf{3 8 , 3 9 ]}\right.$. Let $0<\alpha_{0} \ll \alpha^{*} \ll 1$. Let $u_{0} \in \mathcal{A}$ and $u \in$ $\mathcal{C}\left([0, T), H^{1}\right)$ be the corresponding solution of (1.1). Then, one of the following three scenarios occurs:

(Blow up) For all $t \in[0, T)$, one has $u(t) \in \mathcal{T}_{\alpha^{*}}$ and the solution blows up in finite time $T<+\infty$ with

$$
\left\|u_{x}(t)\right\|_{L^{2}} \sim \frac{\left\|Q^{\prime}\right\|_{L^{2}}}{\ell_{0}(T-t)} \quad \text { as } t \uparrow T \text { for some } \ell_{0}>0 .
$$

(Soliton) The solution is global and

$$
u(t, \cdot+x(t)) \rightarrow Q_{\lambda_{\infty}} \text { in } H_{\mathrm{loc}}^{1} \text { as } t \rightarrow+\infty \text { for }\left|\lambda_{\infty}-1\right|+\left|x^{\prime}(t)-1\right| \lesssim \delta\left(\alpha_{0}\right),
$$

where $\delta\left(\alpha_{0}\right) \rightarrow 0$ as $\alpha_{0} \rightarrow 0$.

(Exit and $S$ dynamics) The solution $u$ exits the tube $\mathcal{T}_{\alpha^{*}}$ at some time $t_{u} \in(0, T)$, and there exist $\lambda_{u}>0$ and $x_{u} \in \mathbb{R}$, such that

$$
\left\|\lambda_{u}^{\frac{1}{2}} u\left(t_{u}, \lambda_{u} x+x_{u}\right)-S\left(t^{*}, x\right)\right\|_{L^{2}} \leq \delta\left(\alpha_{0}\right),
$$

where $\delta\left(\alpha_{0}\right) \rightarrow 0$ as $\alpha_{0} \rightarrow 0$ and where $t^{*}>0$ depends only on $\alpha^{*}$. Moreover, if $S$ scatters at $+\infty$ then $u$ is global and scatters at $+\infty$.

In particular, this indicates that for initial data in $\mathcal{A}$, only one type of blowup is possible. In this paper, we prove that for initial data in $H^{1}$, but with slow decay, different blow-up behaviors are possible close to solitons. This means that the decay assumption in the definition of $\mathcal{A}$ is not a technical one. 


\subsection{Exotic blow-up regimes}

We now consider initial data $u_{0} \notin \mathcal{A}$ in the sense that they display an explicit slow decay on the right. Our main result in this paper says that the blow-up rate $\frac{1}{(T-t)}$, which is universal in $\mathcal{A}$, is not valid anymore for such initial data. Indeed, we produce a wide range of different blow-up rates, including grow up in infinite time.

Theorem 1.1 (Exotic blow-up regimes).

(i) Blow up in finite time: for any $v>\frac{11}{13}$, there exists $u \in C\left(\left(0, T_{0}\right], H^{1}\right)$ solution of (1.1) blowing up at $t=0$ with

$$
\left\|u_{x}(t)\right\|_{L^{2}} \sim t^{-v} \text { as } t \downarrow 0^{+} .
$$

(ii) Grow up in infinite time: there exists $u \in C\left(\left[T_{0},+\infty\right), H^{1}\right)$ solution of (1.1) growing up at $+\infty$ with

$$
\left\|u_{x}(t)\right\|_{L^{2}} \sim e^{t} \text { as } t \rightarrow+\infty .
$$

For any $v>0$, there exists $u \in C\left([0,+\infty), H^{1}\right)$ solution of (1.1) growing up at $+\infty$ with

$$
\left\|u_{x}(t)\right\|_{L^{2}} \sim t^{\nu} \text { as } t \rightarrow+\infty .
$$

Moreover, such solutions can be taken arbitrarily close in $H^{1}$ to the family of solitons.

\section{Comments on Theorem 1.1.}

1. Sharpness of the results in [38,39]. Theorem 1.1 above shows the optimality of the results in $[38,39]$ since it proves that some decay assumption (such as $u_{0} \in \mathcal{A}$ ) is required to obtain a unique stable blow-up rate $1 /(T-t)$. This is in contrast with the nonlinear Schrödinger equation, for which the stable blow-up rate is obtained in $H^{1}$, without additional decay assumption (see [49] and references therein). Note from the proof that the solutions obtained in Theorem 1.1 are expected to be unstable (except maybe for $v<1$ in (1.9)). Indeed, they are constructed using a topogical argument involving two possible directions of instability.

2. It is proved in $[33,44]$ that initial data $u_{0}$ such that $(1.5)$ generate solutions that blow-up in finite or infinite time. The proof is by obstruction and Liouville classification and does not provide any estimate on the blow-up speed. This $H^{1}$ result is also sharp in the sense that from Theorem 1.1, both finite or infinite time blow-up may occur in $H^{1}$. All these results thus complement each other.

3. On the role of tails. As one can see from the proof of Theorem 1.1, the blow-up rate is directly related to the precise behavior of the initial data on the right. In particular, other type of blow-up speeds can be produced by similar arguments by adjusting the tail of the initial data. A similar phenomenon was observed for global in time growing up solutions of the parabolic energy critical harmonic heat flow by Gustafson, Nakanishi and Tsai [16]. In such a paper an explicit formula on the 
growth of the solution at infinity is given directly in terms of the initial data which is conceptually very similar to what we observe for $(\mathrm{gKdV})$.

Recall that continua of blow-up rates were observed in pioneering works by Krieger, Schlag and Tataru [27,28] for energy critical wave problems (see also Donninger and Krieger [6]). We also refer to Fila et al. [13] for a formal approach in the case of the energy critical heat equation. All these results point out that the sole critical topology is not enough to classify the flow near the ground state.

4. On the decay assumption. In [38] (see the definition of $\mathcal{A}$ ), the assumption $\int y^{10} \varepsilon^{2}<1$ is not sharp. In Theorem 1.1, the solution contains a tail of the form $x^{-\theta}$ for $x \gg 1$, where $\theta \in\left(1, \frac{29}{18}\right)$. By now, it is not clear what is the sharp decay assumption on the initial data required to get the stable blow-up rate in [38].

Notation. For $f, g \in L^{2}$, we denote their scalar product by $(f, g)=\int f(x) g(x) d x$. We introduce the generator of the $L^{2}$ scaling symmetry $\Lambda f=\frac{1}{2} f+y f^{\prime}$. We let the linearized operator close to the ground state be

$$
L f=-f^{\prime \prime}+f-5 Q^{4} f .
$$

For a given small constant $0<\alpha^{*} \ll 1, \delta\left(\alpha^{*}\right)$ denotes a small constant with $\delta\left(\alpha^{*}\right) \rightarrow 0$ as $\alpha^{*} \rightarrow 0$. We denote by $\mathbf{1}_{I}$ the characteristic function of the interval $I$.

\subsection{Strategy of the proof}

(i) Definition and role of the slow decaying tail. Given $c_{0} \in \mathbb{R}, x_{0} \gg 1, \theta>1$, we fix a smooth function $f_{0}$ which corresponds to a slowly decaying tail

$$
f_{0}(x)=c_{0} x^{-\theta} \text { for } x>\frac{x_{0}}{2}, \quad f_{0}(x)=0 \text { for } x<\frac{x_{0}}{4},
$$

and $q_{0}$ the solution of

$$
\partial_{t} q_{0}+\partial_{x}\left(\partial_{x}^{2} q_{0}+q_{0}^{5}\right)=0, \quad q_{0}(0, x)=f_{0}(x) .
$$

We then consider the solution of (1.1) with initial data $Q+f_{0}$ and claim that it admits a decomposition of the form

$$
u(t, x)=\frac{1}{\lambda^{\frac{1}{2}}(t)}\left(Q_{b(t)}+\lambda^{\frac{1}{2}}(t) q_{0}(t, x(t)) Y_{0}+\varepsilon\right)\left(t, \frac{x-x(t)}{\lambda(t)}\right)+q_{0}(t, x)
$$

for some

$$
\|\varepsilon(t)\|_{H^{1}} \ll 1,
$$

where $Y_{0}$ is a fixed function (see Lemma 2.1 for the definition of $Y_{0}$ and Proposition 2.4 for the justification of this correction term). An essential feature of the nonlinear $(\mathrm{gKdV})$ flow is that $q_{0}(t, x)$ conserves for $x \gtrsim t$ the slow decay of $f_{0}(x)$ (see 
Lemma 2.3). This tail then acts like an external force on the coupled system of modulation equations driving $(b(t), \lambda(t), x(t))$ and modifies its behavior.

(ii) Dynamical system perturbed by a tail on the right. Let us consider the global renormalized time

$$
\frac{d s}{d t}=\frac{1}{\lambda^{3}}
$$

Then, explicit computations similar to the ones in [38] yield to leading order (neglecting $\varepsilon$ and higher order terms in $(b, \lambda, x))$ the set of coupled modulation equations in the setting of the decomposition (1.15):

$$
\frac{\lambda_{s}}{\lambda}+b=0, \quad x_{s}=\lambda, \quad \frac{d}{d s}\left(\frac{b}{\lambda^{2}}+\frac{4}{\int Q} c_{0} \lambda^{-\frac{3}{2}} x^{-\theta}\right)=0 .
$$

This system is to be compared to the unperturbed one obtained in [38], for $u_{0} \in \mathcal{A}$ (without tail)

$$
\frac{d s}{d t}=\frac{1}{\lambda^{3}}, \quad \frac{\lambda_{s}}{\lambda}+b=0, \quad x_{s}=\lambda, \quad \frac{d}{d s}\left(\frac{b}{\lambda^{2}}\right)=0,
$$

which leads to the universal blow-up regime

$$
\frac{b}{\lambda^{2}}=\ell_{0}, \quad \lambda(t)=\ell_{0}(T-t) \text { for some } \ell_{0}>0 .
$$

We now integrate explicitly (1.17) and fit the parameters of the tail $\left(c_{0}, \theta\right)$ to obtain the blow-up regimes described in Theorem 1.1. Integrating in $s$, we find

$$
\frac{b}{\lambda^{2}}+\frac{4}{\int Q} c_{0} \lambda^{-\frac{3}{2}} x^{-\theta}=\ell_{0},
$$

where $\ell_{0}$ is a constant. We focus on the threshold regime $\ell_{0}=0$ leading to

$$
\frac{\lambda_{s}}{\lambda}+b=0, \quad x_{s}=\lambda, \quad \frac{b}{\lambda^{2}}+\frac{4}{\int Q} c_{0} \lambda^{-\frac{3}{2}} x^{-\theta}=0,
$$

which can now be integrated as

$$
\lambda^{-\frac{1}{2}} \lambda_{s}=\frac{4}{\int Q} c_{0} \lambda x^{-\theta}=\frac{4}{\int Q} c_{0} x_{s} x^{-\theta}
$$

or equivalently, after integration

$$
\lambda^{\frac{1}{2}}(s)+\frac{2}{\int Q} \frac{1}{\theta-1} c_{0} x^{-\theta+1}(s)=\ell_{1} .
$$


We focus again on the threshold regime $\ell_{1}=0$, leading to

$$
\lambda^{\frac{1}{2}}(s)=-\frac{2}{\int Q} \frac{1}{\theta-1} c_{0} x^{-\theta+1}(s) .
$$

We see that $c_{0}<0$ is necessary at this point and

$$
x_{s}(s)=\lambda(s)=\left(\frac{2}{\int Q} \frac{1}{\theta-1} c_{0}\right)^{2} x^{-2 \theta+2}(s) .
$$

By integration on $\left[s_{0}, s\right]$, choosing $x^{2 \theta-1}\left(s_{0}\right)=(2 \theta-1)\left(\frac{2}{\int Q} \frac{1}{\theta-1} c_{0}\right)^{2} s_{0}$, we obtain

$$
x^{2 \theta-1}(s)=(2 \theta-1)\left(\frac{2}{\int Q} \frac{1}{\theta-1} c_{0}\right)^{2} s,
$$

and thus

$$
\lambda(s)=(2 \theta-1)^{\frac{-2 \theta+2}{2 \theta-1}}\left(\frac{2}{\int Q} \frac{1}{\theta-1} c_{0}\right)^{\frac{2}{2 \theta-1}} s^{-\frac{2(\theta-1)}{2 \theta-1}} .
$$

Set

$$
\beta=\frac{2(\theta-1)}{2 \theta-1}, \quad \theta=\frac{1-\frac{\beta}{2}}{1-\beta}, \quad c_{0}=-\frac{\int Q}{2}(\theta-1)(2 \theta-1)^{\theta-1},
$$

so that

$$
\lambda(s)=s^{-\beta}, \quad x(s)=\frac{1}{1-\beta} s^{1-\beta}, \quad b(s)=\frac{\beta}{s} .
$$

Of course, one can check directly that (1.20) are solutions of the system (1.17) but the above computation reveals the two instability directions

$$
\ell_{0}=\ell_{1}=0,
$$

and justifies the use of a topological argument to construct the solution.

(iii) Control of the remainder term. We now aim at constructing an exact solution which corresponds to control the remainder term $\varepsilon(t, x)$. Note that we may now choose $\varepsilon_{0}(x)$ to be well localized on the right, and we therefore adapt the machinery developed in [38] to construct a mixed energy/Virial functional

$$
\mathcal{F} \sim \int \psi \varepsilon_{y}^{2}+\varphi \varepsilon^{2}-\frac{1}{3} \psi\left[\left(Q_{b}+\varepsilon\right)^{6}-Q_{b}^{6}-6 Q_{b}^{5} \varepsilon\right]
$$

for well chosen cut off functions $(\psi, \varphi)$ which are exponentially decaying to the left, and polynomially growing to the right. Roughly speaking, in the above regime (1.20), this functional enjoys two fundamental properties:

- Coercivity:

$$
\mathcal{F} \gtrsim\|\varepsilon\|_{H_{\text {loc }}^{1}}^{2} .
$$


- Lyapounov monotonicity:

$$
\frac{d}{d s}\left\{s^{j} \mathcal{F}\right\}+s^{j}\|\varepsilon\|_{H_{\mathrm{loc}}^{1}}^{2} \lesssim s^{j-4}, \quad j \geq 0 .
$$

Time integration of the monotonicity formula (1.22) in the regime dictated by (1.20) yields sufficient uniform estimates on $\varepsilon$. Therefore, it only remains to adjust the initial parameters $\left(b\left(s_{0}\right), \lambda\left(s_{0}\right)\right)$ in order to asymptotically satisfy the unstable conditions (1.21). This is achieved using a simple topological argument, as in [4] but in a blow-up setting (see also $[5,17,51,62]$ ).

(iv) Conclusion of the proof returning to the original time variable. The above strategy is implemented for all $0<\beta<\frac{11}{20}$. Now we show how the behavior of the parameters (1.20) (see the precise estimates in (3.10)) in renormalized time leads to the scenarios of Theorem 1.1 in the original time $t$ (after possible scaling and time translation to adjust constants).

- Blow up in finite time: for $\frac{1}{3}<\beta<\frac{11}{20}$. From (1.16) and (1.20) we have

$$
\int_{s_{0}}^{+\infty} \lambda^{3}(s) d s=T<+\infty
$$

and the solution $u(t)$ blows up in finite time $T$. Moreover,

$$
T-t=\int_{s(t)}^{+\infty} \lambda^{3}\left(s^{\prime}\right) d s^{\prime} \sim \frac{s^{-(3 \beta-1)}}{3 \beta-1}, \quad \lambda(t) \sim[(3 \beta-1)(T-t)]^{\frac{\beta}{3 \beta-1}},
$$

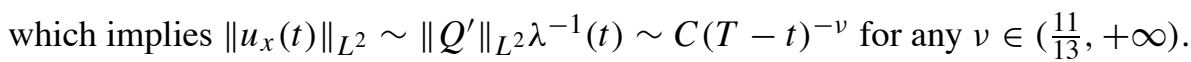

- Grow up in infinite time: for $\beta=\frac{1}{3}$, the solution $u(t)$ is global in time since $\int_{s_{0}}^{+\infty} \lambda^{3}(s) d s=+\infty$. Moreover, for some $c_{0}$ and some $c_{1}>0$,

$$
t=\int_{s_{0}}^{s(t)} \lambda^{3}\left(s^{\prime}\right) d s^{\prime}=\log s+c_{0}+O\left(s^{-\frac{1}{10}}\right), \quad s \sim c_{1} e^{t}, \quad \lambda(t) \sim c_{1}^{-\frac{1}{3}} e^{-\frac{t}{3}} .
$$

This means grow up in infinite time for $u(t)$ with exponential growth. Scaling and time translation lead to any exponential rate $e^{-c t}, c>0$. Finally, for $0<\beta<\frac{1}{3}$, we also obtain a global solution $u(t)$ since

$$
\int_{s_{0}}^{+\infty} \lambda^{3}(s) d s \geq 2^{-3} \int_{s_{0}}^{+\infty} s^{-3 \beta} d s=+\infty
$$

and

$$
t=\int_{s_{0}}^{t(s)} \lambda^{3}\left(s^{\prime}\right) d s^{\prime} \sim \frac{1}{1-3 \beta} s^{1-3 \beta}, \quad \lambda(t) \sim(1-3 \beta)^{\frac{\beta}{1-3 \beta}} t^{\frac{\beta}{1-3 \beta}},
$$

which means grow up rates $t^{\nu}$ at $+\infty$, for any $v=\frac{\beta}{1-3 \beta}>0$. 


\section{Decomposition of the solution}

This section is devoted to the study of the geometric decomposition (1.15), and in particular to the derivation of the modulation equations.

\subsection{Inversion of $L$ and $Q_{b}$ profiles}

Let the functional space $\mathcal{Y}$ be the set of functions $f \in \mathcal{C}^{\infty}(\mathbb{R}, \mathbb{R})$ such that

$$
\forall k \in \mathbb{N}, \exists C_{k}, r_{k}>0, \forall y \in \mathbb{R}, \quad\left|f^{(k)}(y)\right| \leq C_{k}(1+|y|)^{r_{k}} e^{-|y|},
$$

and $L$ be the linearized operator close to $Q$ given by (1.12). We establish the following:

Lemma 2.1 (Invertibility of $L$ ). (i) There exists a unique $Y_{0} \in \mathcal{Y}$, even, such that

$$
L Y_{0}=5 Q^{4}, \quad\left(Q, Y_{0}\right)=-\frac{3}{4} \int Q .
$$

(ii) There exists a unique function $P$ such that $P^{\prime} \in \mathcal{Y}$ and

$$
\begin{gathered}
(L P)^{\prime}=\Lambda Q, \quad \lim _{y \rightarrow-\infty} P(y)=\frac{1}{2} \int Q, \lim _{y \rightarrow+\infty} P(y)=0, \\
(P, Q)=\frac{1}{16}\left(\int Q\right)^{2}>0, \quad\left(P, Q^{\prime}\right)=0 .
\end{gathered}
$$

Proof. Note that the existence and uniqueness of $Y_{0}$ follows readily from standard properties of the operator $L$ (see e.g. [38]). Moreover,

$$
\left(Q, Y_{0}\right)=-\frac{1}{2}\left(L \Lambda Q, Y_{0}\right)=-\frac{1}{2}\left(\Lambda Q, 5 Q^{4}\right)=-\frac{3}{4} \int Q^{5}=-\frac{3}{4} \int Q .
$$

Part (ii) is taken from [38], Proposition 2.2.

A simple consequence of Lemma 2.1 (ii) is the existence of a one-parameter family of approximate self-similar profiles $b \mapsto Q_{b},|b| \ll 1$, which provide the leading order deformation of the ground-state profile $Q=Q_{b=0}$ in the blow-up regimes. More precisely, let $\chi \in \mathcal{C}^{\infty}(\mathbb{R})$ be such that $0 \leq \chi \leq 1, \chi^{\prime} \geq 0$ on $\mathbb{R}$, $\chi \equiv 1$ on $[-1,+\infty), \chi \equiv 0$ on $(-\infty,-2]$, and define

$$
\chi_{b}(y)=\chi\left(|b|^{\gamma} y\right), \quad \gamma=\frac{3}{4} .
$$


The following lemma is proved in [38]:

Lemma 2.2 (Approximate self-similar profiles $Q_{b},[38]$ ). Let

$$
Q_{b}(y)=Q(y)+b \chi_{b}(y) P(y) .
$$

Then:

(i) Estimates on $Q_{b}:$ for all $y \in \mathbb{R}$,

$$
\begin{aligned}
& \left|Q_{b}(y)\right| \lesssim e^{-|y|}+|b|\left(\mathbf{1}_{[-2,0]}\left(|b|^{\gamma} y\right)+e^{-\frac{|y|}{2}}\right), \\
& \left|Q_{b}^{(k)}(y)\right| \lesssim e^{-|y|}+|b| e^{-\frac{|y|}{2}}+|b|^{1+k \gamma} \mathbf{1}_{[-2,-1]}\left(|b|^{\gamma} y\right), \quad \text { for } k \geq 1 .
\end{aligned}
$$

(ii) Equation of $Q_{b}$ : let

$$
-\Psi_{b}=\left(Q_{b}^{\prime \prime}-Q_{b}+Q_{b}^{5}\right)^{\prime}+b \Lambda Q_{b}
$$

then, for all $y \in \mathbb{R}$,

$$
\begin{aligned}
& \left|\Psi_{b}(y)\right| \lesssim|b|^{1+\gamma} \mathbf{1}_{[-2,-1]}\left(|b|^{\gamma} y\right)+b^{2}\left(e^{-\frac{|y|}{2}}+\mathbf{1}_{[-2,0]}\left(|b|^{\gamma} y\right)\right), \\
& \left|\Psi_{b}^{(k)}(y)\right| \lesssim|b|^{1+(k+1) \gamma} \mathbf{1}_{[-2,-1]}\left(|b|^{\gamma} y\right)+b^{2} e^{-\frac{|y|}{2}}, \quad \text { for } k \geq 1 .
\end{aligned}
$$

(iii) Mass and energy properties of $Q_{b}$ :

$$
\begin{aligned}
& \left|\int Q_{b}^{2}-\left(\int Q^{2}+2 b \int P Q\right)\right| \lesssim|b|^{2-\gamma}, \\
& \left|E\left(Q_{b}\right)+b \int P Q\right| \lesssim b^{2} .
\end{aligned}
$$

\subsection{Definition of the tail on the right}

We now introduce the slowly decaying tail on the right. Let $c_{0}<0, x_{0} \gg 1, \theta>1$ and let $f_{0}$ be a smooth function such that

$$
f_{0}(x)= \begin{cases}c_{0} x^{-\theta} & \text { for } x>\frac{x_{0}}{2} \\ 0 & \text { for } x<\frac{x_{0}}{4}\end{cases}
$$

and

$$
\left|\frac{d^{k} f_{0}}{d x^{k}}(x)\right| \lesssim|x|^{-\theta-k}, \quad \forall(x, k) \in \mathbb{R} \times \mathbb{N} .
$$

Let $q_{0}$ be the solution of

$$
\partial_{t} q_{0}+\partial_{x}\left(\partial_{x}^{2} q_{0}+q_{0}^{5}\right)=0, \quad q_{0}(0, x)=f_{0}(x) .
$$


A simple consequence of local energy estimates for $(\mathrm{gKdV})$ is the propagation of the tail on the right:

Lemma 2.3 (Asymptotic behavior of $q_{0}$ ). The solution $q_{0}$ of (2.16) is global, smooth and bounded in $H^{1}$. Moreover, $\forall t \geq 0, \forall x>\frac{t}{2}+\frac{x_{0}}{2}$,

$$
\begin{gathered}
\forall k \geq 0, \quad\left|\partial_{x}^{k} q_{0}(t, x)-f_{0}^{(k)}(x)\right| \lesssim t^{\frac{3}{8}} x^{-\theta-\frac{19}{8}-k} \lesssim x^{-\theta-2-k}, \\
\left|\partial_{t} q_{0}(t, x)\right| \lesssim x^{-\theta-3} .
\end{gathered}
$$

See the proof of Lemma 2.3 in Appendix A.

\subsection{Decomposition of the solution}

Let $c_{0} \in \mathbb{R}, \lambda_{0} \ll 1$ and $x_{0} \gg 1$. Consider a solution $u(t, x)$ of (1.1) and set

$$
w(t, x)=u(t, x)-q_{0}(t, x) .
$$

We assume that $w$ is close to $Q$ in the following sense: there exist $\left(\lambda_{1}(t), x_{1}(t)\right) \in$ $\mathbb{R}_{+}^{*} \times \mathbb{R}$ and $\varepsilon_{1}(t)$ such that

$$
\begin{aligned}
\forall t \in\left[0, t_{0}\right], & \lambda_{1}(t)<\frac{10}{9} \lambda_{0}, \quad x_{1}(t)>\frac{9}{10} x_{0}, \\
w(t, x) & =\frac{1}{\lambda_{1}^{\frac{1}{2}}(t)}\left(Q+\varepsilon_{1}\right)\left(t, \frac{x-x_{1}(t)}{\lambda_{1}(t)}\right)
\end{aligned}
$$

with

$$
\forall t \in\left[0, t_{0}\right], \quad\left\|\varepsilon_{1}(t)\right\|_{L^{2}}+\left(\int\left(\partial_{y} \varepsilon_{1}\right)^{2} e^{-\frac{|y|}{2}} d y\right)^{\frac{1}{2}} \leq \alpha^{*}
$$

for some small enough universal constant $\alpha^{*}>0$. We collect in the following proposition the standard preliminary estimates on this decomposition, and derive in particular the set of modulation equations as a consequence of a suitable choice of orthogonality conditions for the remainder term.

Proposition 2.4 (Preliminary estimates and modulation equations). Assume (2.20)-(2.22) for $\alpha^{*}$ small enough, and assume $x_{0}$ large enough and $\lambda_{0}$ small enough.

(i) Decomposition: There exist $\mathcal{C}^{1}$ functions $(\lambda, x, b):\left[0, t_{0}\right] \rightarrow(0,+\infty) \times \mathbb{R}^{2}$ such that

$$
\forall t \in\left[0, t_{0}\right], \quad \lambda^{\frac{1}{2}}(t) w(t, \lambda(t) y+x(t))=Q_{b(t)}(y)+p(t) Y_{0}(y)+\varepsilon(t, y),
$$

where $Y_{0}$ is given by (2.2),

$$
p(t)=q(t, 0), \quad q(t, y)=\lambda^{\frac{1}{2}}(t) q_{0}(t, \lambda(t) y+x(t)),
$$


and $\varepsilon(t, y)$ satisfies

$$
\begin{aligned}
(\varepsilon(t), y \Lambda Q) & =(\varepsilon(t), \Lambda Q)=(\varepsilon(t), Q)=0, \\
\lambda(t) & <\frac{5}{4} \lambda_{0}, \quad x(t)>\frac{4}{5} x_{0} .
\end{aligned}
$$

(ii) Estimates induced by the conservation laws:

$$
\begin{gathered}
\|\varepsilon(s)\|_{L^{2}}^{2} \lesssim\left|\int u_{0}^{2}-\int Q^{2}\right|+|b(s)|+|p(s)|+x_{0}^{-\theta+\frac{1}{2}}, \\
\frac{1}{\lambda^{2}}\left\|\varepsilon_{y}(s)\right\|_{L^{2}}^{2} \lesssim\left|E\left(u_{0}\right)\right|+\left|\frac{b}{\lambda^{2}}+c_{0} \frac{4}{\int Q} \lambda^{-\frac{3}{2}} x^{-\theta}\right| \\
+\frac{b^{2}}{\lambda^{2}}+\frac{|p|}{x^{2}}+\frac{|p|}{\lambda x}+\frac{p^{2}}{\lambda^{2}}+x_{0}^{-\theta-\frac{1}{2}} .
\end{gathered}
$$

(iii) Modulation equations: Assume

$$
\forall t \in\left[0, t_{0}\right], \quad x(t)>\frac{2}{3} t+\frac{2}{3} x_{0} .
$$

Let $s_{0}>1$ and consider the rescaled time

$$
s=s(t)=s_{0}+\int_{0}^{t} \frac{d t^{\prime}}{\lambda^{3}\left(t^{\prime}\right)} \quad \text { or equivalently } \quad \frac{d s}{d t}=\frac{1}{\lambda^{3}}, \quad s(0)=s_{0} .
$$

Then, on $\left[s_{0}, s\left(t_{0}\right)\right]$,

$$
\begin{aligned}
& \left|\frac{\lambda_{s}}{\lambda}+b\right|+\left|\frac{x_{s}}{\lambda}-1\right| \lesssim\left(\int \varepsilon^{2} e^{-\frac{|y|}{10}}\right)^{\frac{1}{2}}+b^{2}+p^{2}+\frac{\lambda}{x}|p|, \\
& \left|b_{s}\right| \lesssim \int \varepsilon^{2} e^{-\frac{|y|}{10}}+|p|\left(\int \varepsilon^{2} e^{-\frac{|y|}{10}}\right)^{\frac{1}{2}}+|b|^{2}+|b||p|+p^{4}+\frac{\lambda}{x}|p|, \\
& \left|\frac{d}{d s}\left(\frac{b}{\lambda^{2}}+\frac{4}{\left(\int Q\right)} c_{0} \lambda^{-\frac{3}{2}} x^{-\theta}\right)\right| \\
& \lesssim \frac{1}{\lambda^{2}}\left(|b|^{3}+|p|^{3}+(|b|+|p|)\left(\int \varepsilon^{2} e^{-\frac{|y|}{10}}\right)^{\frac{1}{2}}+\int \varepsilon^{2} e^{-\frac{|y|}{10}}+\frac{\lambda^{2}}{x^{2}}|p|+\frac{\lambda}{x^{3}}|p|\right) .
\end{aligned}
$$

Remark 2.5. The bounds (2.31)-(2.33) will justify the dynamical system (1.17).

Proof. Step 1. Proof of (i). This is a standard modulation claim. As usual, the decomposition is first performed for a fixed time $t$. For $t \in\left[0, t_{0}\right]$ fixed, define the map

$$
\Theta:\left(\bar{b}, \bar{\lambda}, \bar{x}, w, z_{0}\right) \mapsto\left(\int Q \bar{\varepsilon}, \int \Lambda Q \bar{\varepsilon}, \int Q^{\prime} \bar{\varepsilon}\right)
$$


where $x_{0}=1 / z_{0}$,

$$
\begin{aligned}
\bar{\varepsilon}(y) & =\varepsilon_{\left(\bar{b}, \bar{\lambda}, \bar{x}, w_{1}, z_{0}\right)}(y) \\
& =\bar{\lambda}^{\frac{1}{2}} w_{1}(t, \bar{\lambda} y+\bar{x})-\bar{\lambda}^{\frac{1}{2}} \lambda_{1}^{\frac{1}{2}}(t) q_{0}\left(t, x_{1}(t)+\bar{x}\right) Y_{0}(y)-Q_{\bar{b}}(y), \\
w_{1}(t, y) & =\lambda_{1}(t)^{\frac{1}{2}} w\left(t, \lambda_{1}(t) y+x_{1}(t)\right)=Q(y)+\varepsilon_{1}(t, y) .
\end{aligned}
$$

We have $\left.\bar{\varepsilon}\right|_{(0,1,0, Q, 0)}=0$, so that $\Theta(0,1,0, Q, 0)=0$ and

$$
\left.\partial_{\bar{b}} \bar{\varepsilon}\right|_{(0,1,0, Q, 0)}=P,\left.\quad \partial_{\bar{\lambda}} \bar{\varepsilon}\right|_{(0,1,0, Q, 0)}=\Lambda Q,\left.\quad \partial_{\bar{x}} \bar{\varepsilon}\right|_{(0,1,0, Q, 0)}=Q^{\prime} .
$$

so that differentiating the map $\Theta$ with respect to the variables $(\bar{b}, \bar{\lambda}, \bar{x})$ at the point $(0,1,0, Q, 0)$ we find the Jacobian matrix

$$
\left(\begin{array}{ccc}
(P, Q) & (P, \Lambda Q) & \left(P, Q^{\prime}\right) \\
(\Lambda Q, Q) & (\Lambda Q, \Lambda Q) & \left(\Lambda Q, Q^{\prime}\right) \\
\left(Q^{\prime}, Q\right) & \left(Q^{\prime}, \lambda Q\right) & \left(Q^{\prime}, Q^{\prime}\right)
\end{array}\right)=\left(\begin{array}{ccc}
(P, Q) & (P, \Lambda Q) & 0 \\
0 & (\Lambda Q, \Lambda Q) & 0 \\
0 & 0 & \left(Q^{\prime}, Q^{\prime}\right)
\end{array}\right)
$$

which is not degenerate since $(P, Q)>0$. It follows from these observations that we can apply the implicit function theorem to $\Theta$ : for $w_{1}$ small and $x_{0}$ large, there exists a unique $(\bar{b}, \bar{\lambda}, \bar{x})=(\bar{b}, \bar{\lambda}, \bar{x})\left(w_{1}, x_{0}\right)$ close to $(0,1,0)$ such that $\Theta\left(\bar{b}, \bar{\lambda}, \bar{x}, w_{1}, \frac{1}{x_{0}}\right)=0$. Then, we define $b(t)=\bar{b}\left(w_{1}(t), x_{0}\right), \lambda(t)=\bar{\lambda}\left(w_{1}(t), x_{0}\right) \lambda_{1}(t)$, $x(t)=\bar{x}\left(w_{1}(t), x_{0}\right)+x_{1}(t)$ and $\varepsilon(t)=\bar{\varepsilon}(t)$. The regularity of $(b(t), \lambda(t), x(t))$ now follow from standard arguments. It follows that we have the following decomposition of $u(t, x)$ :

$$
\begin{aligned}
u(t, x) & =\frac{1}{\lambda^{\frac{1}{2}}(t)}\left(Q_{b(t)}+p(t) Y_{0}+\varepsilon\right)\left(t, \frac{x-x(t)}{\lambda(t)}\right)+q_{0}(t, x) \\
& =\frac{1}{\lambda^{\frac{1}{2}}(t)}\left(Q_{b(t)}+p(t) Y_{0}+\varepsilon+q\right)\left(t, \frac{x-x(t)}{\lambda(t)}\right) .
\end{aligned}
$$

Step 2. Equation of $\varepsilon$ and a priori bounds. To write the equation of $\varepsilon$, we first derive the equation of $w$ from the equations of $u(t)$ and $q_{0}(t)$, getting

$$
w_{t}+\left(w_{x x}+w^{5}\right)_{x}=-\left(W_{0}\right)_{x},
$$

where

$$
W_{0}=5 w^{4} q_{0}+10 w^{3} q_{0}^{2}+10 w^{2} q_{0}^{3}+5 w q_{0}^{4} .
$$

Second, set $\varepsilon_{Y}(s, y)=p(s) Y_{0}(y)+\varepsilon(s, y)$ so that

$$
w(s, x)=\frac{1}{\lambda^{\frac{1}{2}}(s)}\left(Q_{b(s)}+\varepsilon_{Y}\right)\left(s, \frac{x-x(s)}{\lambda(s)}\right) .
$$


By standard computations, we obtain for $\varepsilon_{Y}$

$$
\begin{aligned}
\partial_{s} \varepsilon_{Y}= & \left(-\partial_{y}^{2} \varepsilon_{Y}+\varepsilon_{Y}-\left(\varepsilon_{Y}+Q_{b}\right)^{5}+Q_{b}^{5}\right)_{y}-\left(5 Q^{4} q\right)_{y}+\frac{\lambda_{s}}{\lambda} \Lambda \varepsilon_{Y} \\
& +\left(\frac{\lambda_{s}}{\lambda}+b\right) \Lambda Q_{b}+\left(\frac{x_{s}}{\lambda}-1\right)\left(Q_{b}+\varepsilon_{Y}\right)_{y}+\Phi_{b}+\Psi_{b}-W_{y}
\end{aligned}
$$

where

$$
W=5\left(Q_{b}+\varepsilon_{Y}\right)^{4} q-5 Q^{4} q+10\left(Q_{b}+\varepsilon_{Y}\right)^{3} q^{2}+10\left(Q_{b}+\varepsilon_{Y}\right)^{2} q^{3}+5\left(Q_{b}+\varepsilon_{Y}\right) q^{4} .
$$

Finally, we replace $\varepsilon(s, y)=\varepsilon_{Y}(s, y)-p(s) Y_{0}(y)$ and use $L Y_{0}=5 Q^{4}$, to obtain

$$
\begin{aligned}
\partial_{s} \varepsilon= & \left(-\partial_{y}^{2} \varepsilon+\varepsilon-\left(\varepsilon+p Y_{0}+Q_{b}\right)^{5}+Q_{b}^{5}+p 5 Q^{4} Y_{0}\right)_{y} \\
& -p_{s} Y_{0}+\left(5 Q^{4}(p-q)\right)_{y}+\frac{\lambda_{s}}{\lambda}\left(\Lambda \varepsilon+p \Lambda Y_{0}\right)+\left(\frac{\lambda_{s}}{\lambda}+b\right) \Lambda Q_{b} \\
& +\left(\frac{x_{s}}{\lambda}-1\right)\left(Q_{b}+\varepsilon+p Y_{0}\right)_{y}+\Phi_{b}+\Psi_{b}-W_{y},
\end{aligned}
$$

and

$$
\begin{aligned}
W= & 5\left(Q_{b}+\varepsilon+p Y_{0}\right)^{4} q-5 Q^{4} q+10\left(Q_{b}+\varepsilon+p Y_{0}\right)^{3} q^{2} \\
& +10\left(Q_{b}+\varepsilon+p Y_{0}\right)^{2} q^{3}+5\left(Q_{b}+\varepsilon+p Y_{0}\right) q^{4}
\end{aligned}
$$

We now claim the following bounds which we will be used along the proof:

Claim 2.6. (a) Estimates on $q(s)$.

$$
\|q(s)\|_{L^{2}} \lesssim x_{0}^{-\theta+\frac{1}{2}},\left\|q_{y}(s)\right\|_{L^{2}} \lesssim \lambda(s) x_{0}^{-\theta-\frac{1}{2}},\|q(s)\|_{L^{\infty}} \lesssim \lambda^{\frac{1}{2}}(s) x_{0}^{-\theta} .
$$

(b) Properties of the function $p(s)$ :

$$
\begin{gathered}
\left|p(s)-c_{0} \lambda^{\frac{1}{2}}(s) x^{-\theta}(s)\right| \lesssim c_{0} \lambda^{\frac{1}{2}}(s) x^{-\theta-2}(s) \lesssim x^{-2}(s)|p(s)|, \\
e^{-\frac{3|y|}{4}}|p(s)-q(s, y)| \lesssim \frac{\lambda(s)}{x(s)}|p(s)| e^{-\frac{|y|}{4}} \\
\left|\left(\left(5 Q^{4}(p-q)\right)_{y}, Q\right)-c_{0}\left(\int Q\right) \theta \lambda^{-\frac{3}{2}} x^{-\theta-1}\right| \lesssim \frac{\lambda^{2}(s)}{x^{2}(s)}|p(s)|+\frac{\lambda(s)}{x^{3}(s)}|p(s)|, \\
\left|p_{s}-\frac{1}{2} \frac{\lambda_{s}}{\lambda} p+\theta \frac{x_{s}}{x} p\right| \leq \frac{\lambda}{x^{3}}|p|\left(\left|\frac{x_{s}}{\lambda}-1\right|+1\right) \\
\left|p_{s}\right| \lesssim\left|\frac{\lambda_{s}}{\lambda}\right||p|+\frac{\lambda}{x}|p|\left(\left|\frac{x_{s}}{\lambda}-1\right|+1\right)
\end{gathered}
$$


(c) Estimates for the remainder term $W$ : let

$$
\begin{aligned}
\widetilde{W}= & 5\left(Q_{b}+\varepsilon+p Y_{0}\right)^{4} p-5 Q^{4} p+10\left(Q_{b}+\varepsilon+p Y_{0}\right)^{3} p^{2} \\
& +10\left(Q_{b}+\varepsilon+p Y_{0}\right)^{2} p^{3}+5\left(Q_{b}+\varepsilon+p Y_{0}\right) p^{4}
\end{aligned}
$$

Then,

$$
\begin{gathered}
\int|W-\widetilde{W}| e^{-\frac{3}{4}|y|} \lesssim\left(|p|+|b|+\left(\int \varepsilon^{2} e^{-\frac{|y|}{10}}\right)^{\frac{1}{2}}\right) \frac{\lambda}{x}|p| \\
\left|\left((\widetilde{W})_{y}, \Lambda Q\right)\right|+\left|\left((\widetilde{W})_{y}, Q\right)\right| \lesssim b^{2}+p^{8}+|b||p|+|p|\left(\int \varepsilon^{2} e^{-\frac{|y|}{10}}\right)^{\frac{1}{2}} \\
+\int \varepsilon^{2} e^{-\frac{|y|}{10}}, \\
\left|\left((\widetilde{W})_{y}, y \Lambda Q\right)\right| \lesssim \int \varepsilon^{2} e^{-\frac{|y|}{10}}+b^{2}+p^{2} .
\end{gathered}
$$

Proof of Claim 2.6.

(a). Since $q_{0}(t)$ is solution of (1.1), for all $t$,

$$
\left\|q_{0}(t)\right\|_{L^{2}}=\left\|f_{0}\right\|_{L^{2}} \lesssim x_{0}^{-\theta+\frac{1}{2}}, \quad E\left(q_{0}(t)\right)=E\left(f_{0}\right) \lesssim x_{0}^{-\theta-\frac{1}{2}}
$$

and (2.40) follows.

(b). Since $p(s)=\lambda^{\frac{1}{2}}(s) q_{0}(s, x(s))$, (2.41) follows from (2.29) and (2.17). In particular, since $c_{0}<0$, we have $p<0$.

Next, by (2.29), (2.17) and (2.15), splitting the two cases $\lambda|y|<\frac{x(s)}{4}$ and $\lambda|y|>\frac{x(s)}{4}$, we have

$$
\begin{aligned}
& e^{-\frac{3|y|}{4}}|p(s)-q(s, y)|=e^{-\frac{3|y|}{4}} \lambda^{\frac{1}{2}}(s)\left|q_{0}(s, x(s))-q_{0}(s, \lambda(s) y+x(s))\right| \\
& \lesssim|y| e^{-\frac{|y|}{2}} \lambda^{\frac{3}{2}}(s)\left(e^{-\frac{|y|}{4}}\left\|q_{0}^{\prime}(s)\right\|_{L^{\infty}\left(x>\frac{3}{4} x(s)\right)}+e^{-\frac{x(s)}{16 \lambda(s)}}\left\|q_{0}^{\prime}(s)\right\|_{L^{\infty}}\right) \\
& \lesssim c_{0} e^{-\frac{|y|}{4}} \lambda^{\frac{3}{2}}(s)\left(x^{-\theta-1}(s)+e^{-\frac{x(s)}{16 \lambda(s)}} x_{0}^{-\theta-1}\right) \\
& \lesssim c_{0} e^{-\frac{|y|}{4}} \lambda^{\frac{3}{2}}(s) x^{-\theta-1}(s) \lesssim \frac{\lambda}{x}|p| e^{-\frac{|y|}{4}} .
\end{aligned}
$$


Next, by (2.29) and (2.17),

$$
\begin{aligned}
& \left(\left(5 Q^{4}(p-q)\right)_{y}, Q\right)=-5 \int Q^{4} Q_{y}(p-q)=-\int Q^{5} q_{y} \\
& =-\lambda^{\frac{3}{2}}(s) \int Q^{5}(y) \partial_{x} q_{0}(s, \lambda(s) y+x(s)) d y \\
& =-\lambda^{\frac{3}{2}} f_{0}^{\prime}(x(s)) \int Q^{5}-\lambda^{\frac{3}{2}} \int Q^{5}\left(f_{0}^{\prime}(\lambda y+x(s))-f_{0}^{\prime}(x(s))\right) d y \\
& \quad-\lambda^{\frac{3}{2}}(s) \int Q^{5}(y)\left(\partial_{x} q_{0}(s, \lambda(s) y+x(s))-f_{0}^{\prime}(\lambda y+x(s))\right) d y \\
& =c_{0}\left(\int Q\right) \theta \lambda^{\frac{3}{2}}(s) x^{-\theta-1}(s)+O\left(\lambda^{\frac{3}{2}} e^{-\frac{5}{4} x(s)}\right) \\
& \quad+O\left(\lambda^{\frac{5}{2}} x^{-\theta-2}\right)+O\left(\lambda^{\frac{3}{2}} x^{-\theta-3}(s)\right) \\
& =c_{0}\left(\int Q\right) \theta \lambda^{\frac{3}{2}}(s) x^{-\theta-1}(s)+O\left(\frac{\lambda^{2}}{x^{2}}|p|\right)+O\left(\frac{\lambda}{x^{3}}|p|\right)
\end{aligned}
$$

where we have split the integrals above into $|y|>\frac{1}{4} x(s)$ and $|y|<\frac{1}{4} x(s)$ and using the fact that for $|y|<\frac{1}{4} x(s), \lambda y+x(s)>x(s)-|y|>\frac{3}{4} x(s)>\frac{1}{2} t+\frac{1}{2} x_{0}$, so that (2.17) holds for $x=\lambda y+x(s)$, and (2.43) is proved.

Now, we prove (2.44). By explicit differentiation and Lemma 2.3,

$$
\begin{aligned}
p_{s}-\frac{1}{2} \frac{\lambda_{s}}{\lambda} p & =\lambda^{\frac{1}{2}}\left(q_{0}\right)_{s}(s, x(s))+\lambda^{\frac{1}{2}} x_{s} \partial_{x} q_{0}(s, x(s)) \\
& =\lambda^{\frac{7}{2}}\left(q_{0}\right)_{t}(s, x(s))+\lambda^{\frac{1}{2}} x_{s} f_{0}^{\prime}(x(s))+O\left(\frac{\lambda}{x^{3}}|p|\left(\left|\frac{x_{s}}{\lambda}-1\right|+1\right)\right) \\
& =-\theta \frac{x_{s}}{x}|p|+O\left(\frac{\lambda}{x^{3}}|p|\left(\left|\frac{x_{s}}{\lambda}-1\right|+1\right)\right) .
\end{aligned}
$$

Since $\left|x_{s}\right| \leq \lambda\left(\left|\frac{x_{s}}{\lambda}-1\right|+1\right),(2.45)$ follows.

(c). For (2.46), we first note

$$
\begin{aligned}
\widetilde{W}-W= & 5\left[\left(Q_{b}+\varepsilon+p Y_{0}\right)^{4}-Q^{4}\right](p-q)+10\left(Q_{b}+\varepsilon+p Y_{0}\right)^{3}\left(p^{2}-q^{2}\right) \\
& +10\left(Q_{b}+\varepsilon+p Y_{0}\right)^{2}\left(p^{3}-q^{3}\right)+5\left(Q_{b}+\varepsilon+p Y_{0}\right)\left(p^{4}-q^{4}\right) .
\end{aligned}
$$


Thus,

$$
\begin{aligned}
& \int e^{-\frac{3}{4}|y|}|W-\widetilde{W}| \\
& \lesssim(|p|+|b|) \int e^{-\frac{3}{4}|y|}|p-q|+\left(\int \varepsilon^{2} e^{-\frac{|y|}{10}}\right)^{\frac{1}{2}}\left(\int e^{-\left(\frac{3}{2}-\frac{1}{10}\right)|y|}|p-q|^{2}\right)^{\frac{1}{2}} \\
& \quad+\int e^{-\frac{3}{4}|y|}\left|p^{2}-q^{2}\right| \lesssim\left(|p|+|b|+\left(\int \varepsilon^{2} e^{-\frac{|y|}{10}}\right)^{\frac{1}{2}}\right) \frac{\lambda}{x}|p|,
\end{aligned}
$$

using (2.42), and the following similar estimate

$$
e^{-\frac{3}{4}|y|}\left|p^{2}-q^{2}\right| \lesssim \frac{\lambda}{x} p^{2} e^{-\frac{|y|}{4}}
$$

Next, (2.47) follows from the parity properties and then direct estimates. (2.48) follows from direct estimates. Note that $p^{2}$ appears in (2.48) because there is no cancellation due to parity for this term. This concludes the proof of Claim 2.6.

Step 3. Estimates induced by the conservation laws. By $L^{2}$ norm conservation,

$$
\begin{aligned}
\int u^{2}(0)-\int Q^{2}= & \int Q_{b}^{2}-\int Q^{2}+\int\left(\varepsilon+p Y_{0}+q\right)^{2}+2 \int\left(\varepsilon+p Y_{0}+q\right) Q_{b} \\
= & 2 b(P, Q)+O\left(|b|^{2-\gamma}\right)+\|\varepsilon\|_{L^{2}}^{2}+O\left(|b|^{1-\frac{\gamma}{2}}\|\varepsilon\|_{L^{2}}\right) \\
& +O\left(|p|+\|q\|_{L^{2}}\right) .
\end{aligned}
$$

Estimate (2.27) follows. By energy conservation, $Q^{\prime \prime}+Q^{5}=Q$ and $\int \varepsilon Q=0$,

$$
\begin{aligned}
& 2 \lambda^{2} E\left(u_{0}\right)=2 E\left(Q_{b}+\varepsilon+p Y_{0}+q\right) \\
& =2 E\left(Q_{b}\right)-2 \int\left(\varepsilon+p Y_{0}+q\right)\left(\left(Q_{b}-Q\right)_{y y}+\left(Q_{b}^{5}-Q^{5}\right)\right)-2 \int\left(p Y_{0}+q\right) Q \\
& \quad+\int\left(\varepsilon+p Y_{0}+q\right)_{y}^{2}-\frac{1}{3} \int\left(\left(Q_{b}+\varepsilon+p Y_{0}+q\right)^{6}-Q_{b}^{6}-6 Q_{b}^{5}(\varepsilon+p Y+q)\right) \\
& =-2 b(P, Q)+O\left(b^{2}\right)+O\left(|b|^{1+\frac{3}{2} \gamma}\left(\|\varepsilon\|_{L^{2}}+|p|+\|q\|_{L^{2}}\right)\right) \\
& \quad-2 p\left(\int Y_{0} Q+\int Q\right)+2 \int(p-q) Q+\left\|\varepsilon_{y}\right\|_{L^{2}}^{2}+O\left(p^{2}\right) \\
& \quad+O\left(\left\|\varepsilon_{y}\right\|_{L^{2}}\left(|p|+\left\|q_{y}\right\|_{L^{2}}\right)\right)+2 \lambda^{2} E\left(q_{0}\right) \\
& \quad-\frac{1}{3} \int\left(\left(Q_{b}+\varepsilon+p Y_{0}+q\right)^{6}-Q_{b}^{6}-6 Q_{b}^{5}\left(\varepsilon+p Y_{0}+q\right)-q^{6}\right) .
\end{aligned}
$$


By (2.2), we have $\int Y_{0} Q+\int Q=\frac{1}{4} \int Q$. Using in addition (2.4), (2.42), (2.41), we obtain

$$
\begin{aligned}
\frac{1}{\lambda^{2}}\left\|\varepsilon_{y}\right\|_{L^{2}}^{2} \lesssim & \left|E\left(u_{0}\right)\right|+\left|\frac{1}{4} \frac{b}{\lambda^{2}} \int Q+\frac{p}{\lambda^{2}}\right|+\frac{b^{2}}{\lambda^{2}}+\frac{|b|^{1+\frac{3}{2} \gamma}}{\lambda^{2}}\left(\|\varepsilon\|_{L^{2}}+|p|+\|q\|_{L^{2}}\right) \\
& +\frac{|p|}{\lambda x}+\frac{p^{2}}{\lambda^{2}}+x_{0}^{-\theta-\frac{1}{2}} \\
\lesssim & \left|E\left(u_{0}\right)\right|+\left|\frac{1}{4} \frac{b}{\lambda^{2}} \int Q+c_{0} \lambda^{-\frac{3}{2}} x^{-\theta}\right|+\frac{b^{2}}{\lambda^{2}} \\
& +\frac{|p|}{x^{2}}+\frac{|p|}{\lambda x}+\frac{p^{2}}{\lambda^{2}}+x_{0}^{-\theta-\frac{1}{2}}
\end{aligned}
$$

Step 4. Modulation equations. We argue as in [38], proof of Lemma 2.7, differentiating with respect to $s$ the orthogonality conditions $\int \varepsilon \Lambda Q=0, \int \varepsilon Q^{\prime}=0$ and $\int \varepsilon Q=0$ and using (2.39) to obtain (2.31) and (2.32). Here, we will treat only the terms coming from $q$ and $p Y_{0}$ in (2.39) and we refer the reader to [38] for more details on the other terms.

Proof of (2.31) and (2.32). It follows from computations in [38, proof of Lemma 2.7] and Claim 2.6 that

$$
\begin{aligned}
& \left|\left(\frac{\lambda_{s}}{\lambda}+b\right)-\frac{\left(\varepsilon, L(\Lambda Q)^{\prime}\right)}{\|\Lambda Q\|_{L^{2}}^{2}}\right| \\
& \lesssim\left(\left|\frac{\lambda_{s}}{\lambda}+b\right|+|b|\right)\left(|p|+|b|+\left(\int \varepsilon^{2} e^{-\frac{|y|}{10}}\right)^{\frac{1}{2}}\right)+|p|\left(\int \varepsilon^{2} e^{-\frac{|y|}{10}}\right)^{\frac{1}{2}} \\
& \quad+\left|\frac{x_{s}}{\lambda}-1\right|\left(|b|+\left(\int \varepsilon^{2} e^{-\frac{|y|}{10}}\right)^{\frac{1}{2}}\right)+\left|p_{s}\right|+\left|b_{s}\right|+\int \varepsilon^{2} e^{-\frac{|y|}{10}}+p^{8}+\frac{\lambda}{x}|p| .
\end{aligned}
$$

We proceed similarly for $\frac{x_{s}}{\lambda}-1$, taking into account different cancellations due to parity properties

$$
\begin{aligned}
& \left|\left(\frac{x_{s}}{\lambda}-1\right)-\frac{\left(\varepsilon, L(y \Lambda Q)^{\prime}\right)}{\|\Lambda Q\|_{L^{2}}^{2}}\right| \\
& \lesssim\left(\left|\frac{\lambda_{s}}{\lambda}+b\right|+\left|\frac{x_{s}}{\lambda}-1\right|+|b|\right)\left(|p|+|b|+\left(\int \varepsilon^{2} e^{-\frac{|y|}{10}}\right)^{\frac{1}{2}}\right) \\
& \quad+\left|b_{s}\right|+\int \varepsilon^{2} e^{-\frac{|y|}{10}}+p^{2}+\frac{\lambda}{x}|p| .
\end{aligned}
$$


Then, taking the scalar product of (2.39) by $Q$ and arguing similarly, we have the following rough estimate for $b_{s}$ :

$$
\begin{aligned}
\left|b_{s}\right| \lesssim & \left|\frac{\lambda_{s}}{\lambda}+b\right|^{2}+\left|\frac{x_{s}}{\lambda}-1\right|^{2}+|b|^{2}+\int \varepsilon^{2} e^{-\frac{|y|}{10}}+|p|\left(\int \varepsilon^{2} e^{-\frac{|y|}{10}}\right)^{\frac{1}{2}} \\
& +\left|p_{s}\right|+|b||p|+p^{8}+\frac{\lambda}{x}|p| .
\end{aligned}
$$

Combining these estimates with (from (2.44))

$$
\begin{aligned}
\left|p_{s}\right| & \leq\left|\frac{\lambda_{s}}{\lambda}\right||p|+\frac{\lambda}{x}|p|\left(\left|\frac{x_{s}}{\lambda}-1\right|+1\right) \\
& \leq|b||p|+\left|\frac{\lambda_{s}}{\lambda}+b\right||p|+\frac{\lambda}{x}|p|\left(\left|\frac{x_{s}}{\lambda}-1\right|+1\right),
\end{aligned}
$$

from (2.45), we obtain (2.31) and (2.32).

Proof of (2.33). First, we derive a refined equation for $b_{s}$, taking the scalar product of equation (2.39) by $Q$ and proceeding as in [38, proof of Lemma 2.7].

Recall from [38],

$$
\left(\Psi_{b}, Q\right)=-\frac{b^{2}}{8}\|Q\|_{L^{1}}^{2}+O\left(|b|^{3}\right), \quad\left(\Phi_{b}, Q\right)=-\frac{b_{s}}{16}\|Q\|_{L^{1}}^{2}+O\left(|b|^{10}\right) .
$$

Note also that from direct computations and parity properties

$$
\begin{aligned}
& \left|\left(\left(\left(\varepsilon+p Y_{0}+Q_{b}\right)^{5}-Q_{b}^{5}-p 5 Q^{4} Y_{0}-5 Q^{4} \varepsilon\right)_{y}, Q\right)-20 b p\left(\left(Q^{3} Y_{0} P\right)_{y}, Q\right)\right| \\
& \quad+\left|\left((\widetilde{W})_{y}, Q\right)-20 b p\left(\left(P Q^{3}\right)_{y}, Q\right)\right| \\
& \lesssim|b|^{3}+|p|^{3}+\int \varepsilon^{2} e^{-\frac{|y|}{10}}+(|p|+|b|)\left(\int \varepsilon^{2} e^{-\frac{|y|}{10}}\right)^{\frac{1}{2}} .
\end{aligned}
$$

(See [38] for details on the nonlinear terms in $\varepsilon$.) Using (2.43) and the above estimates we find

$$
\begin{aligned}
& \mid b_{s}+2 b^{2}-\frac{16}{\left(\int Q\right)^{2}}(-p_{s}\left(Y_{0}, Q\right)-\frac{\lambda_{s}}{\lambda} p\left(Y_{0}, \Lambda Q\right) \\
&-b p\left[20\left(\left(Q^{3} Y_{0} P\right)_{y}, Q\right)+20\left(\left(P Q^{3}\right)_{y}, Q\right)\right] \\
&\left.+c_{0} \theta\left(\int Q\right) \lambda^{\frac{3}{2}}(s) x^{-\theta-1}(s)\right) \mid \\
& \lesssim|b|^{3}+|p|^{3}+\int \varepsilon^{2} e^{-\frac{|y|}{10}}+(|p|+|b|)\left(\int \varepsilon^{2} e^{-\frac{|y|}{10}}\right)^{\frac{1}{2}}+\frac{\lambda^{2}}{x^{2}}|p|+\frac{\lambda}{x^{3}}|p| .
\end{aligned}
$$


We claim the following cancellation

$$
-\left(Y_{0}, \Lambda Q\right)+20\left(\left(Q^{3} Y_{0} P\right)_{y}, Q\right)+20\left(\left(P Q^{3}\right)_{y}, Q\right)=0
$$

Indeed, $L\left(P^{\prime}\right)=(L P)^{\prime}+20 Q^{3} Q^{\prime} P=\Lambda Q+20 Q^{3} Q^{\prime} P$, and so from (2.3),

$$
\begin{aligned}
& -\left(Y_{0}, \Lambda Q\right)+20\left(\left(Q^{3} Y_{0} P\right)_{y}, Q\right)+20\left(\left(P Q^{3}\right)_{y}, Q\right) \\
& =-\left(Y_{0}, \Lambda Q\right)-20\left(Y_{0}+1, P Q^{3} Q^{\prime}\right) \\
& =-\left(Y_{0}+1, L\left(P^{\prime}\right)\right)+\int \Lambda Q=-\left(L\left(Y_{0}+1\right), P^{\prime}\right)-\frac{1}{2} \int Q \\
& =-\int P^{\prime}-\frac{1}{2} \int Q=P(-\infty)-\frac{1}{2} \int Q=0 .
\end{aligned}
$$

Thus,

$$
\begin{aligned}
& \mid- \frac{\lambda_{s}}{\lambda} p\left(Y_{0}, \Lambda Q\right)+b p\left[20\left(\left(Q^{3} Y_{0} P\right)_{y}, Q\right)+20\left(\left(P Q^{3}\right)_{y}, Q\right)\right] \mid \\
& \quad=|p|\left|\frac{\lambda_{s}}{\lambda}+b\right|\left|\left(Y_{0}, \Lambda Q\right)\right| .
\end{aligned}
$$

Now, from $\left(Y_{0}, Q\right)=-\frac{3}{4} \int Q($ see (2.2)), using (2.41) and (2.44) we note that

$$
\begin{aligned}
& \frac{16}{\left(\int Q\right)^{2}}\left(-p_{s}\left(Y_{0}, Q\right)+c_{0} \theta\left(\int Q\right) \lambda^{\frac{3}{2}}(s) x^{-\theta-1}(s)\right) \\
& =\frac{12}{\left(\int Q\right)} c_{0} \lambda^{\frac{1}{2}} x^{-\theta}\left(\frac{1}{2} \frac{\lambda_{s}}{\lambda}+\frac{\theta}{3} \frac{x_{s}}{x}\right)+O\left(\frac{\lambda}{x^{3}}|p|\right)+O\left(\frac{\lambda}{x}|p|\left|\frac{x_{s}}{\lambda}-1\right|\right) .
\end{aligned}
$$

Therefore,

$$
\begin{aligned}
& \left|b_{s}+2 b^{2}-\frac{4}{\left(\int Q\right)} c_{0} \lambda^{\frac{1}{2}} x^{-\theta}\left(\frac{3}{2} \frac{\lambda_{s}}{\lambda}+\theta \frac{x_{s}}{x}\right)\right| \\
& \lesssim|b|^{3}+|p|^{3}+\int \varepsilon^{2} e^{-\frac{|y|}{10}}+(|p|+|b|)\left(\int \varepsilon^{2} e^{-\frac{|y|}{10}}\right)^{\frac{1}{2}}+\frac{\lambda^{2}}{x^{2}}|p|+\frac{\lambda}{x^{3}}|p| .
\end{aligned}
$$

Now we prove (2.33). By direct computation,

$$
\begin{gathered}
\frac{d}{d s}\left(\frac{4}{\left(\int Q\right)} c_{0} \theta \lambda^{-\frac{3}{2}}(s) x^{-\theta}\right)=-\frac{4}{\left(\int Q\right)} c_{0} \lambda^{-\frac{3}{2}} x^{-\theta}\left(\frac{3}{2} \frac{\lambda_{s}}{\lambda}+\theta \frac{x_{s}}{x}\right) \\
\frac{d}{d s}\left(\frac{b}{\lambda^{2}}\right)=\frac{b_{s}}{\lambda^{2}}-2 \frac{\lambda_{s}}{\lambda} \frac{b}{\lambda^{2}}=\frac{b_{s}}{\lambda^{2}}+2 \frac{b^{2}}{\lambda^{2}}-2\left(\frac{\lambda_{s}}{\lambda}+b\right) \frac{b}{\lambda^{2}} .
\end{gathered}
$$

and (2.33) follows from (2.54) and (2.31). 


\section{Proof of Theorem 1.1}

This section is devoted to the proof of Theorem 1.1, which follows from the modulation equations of Proposition 2.4 coupled with the control of the well localized error $\varepsilon$ as in [38]. We present the new dynamical arguments and postpone the proofs of two technical lemmas adapted from [38] to the Appendices.

\subsection{The bootstrap argument}

Let

$$
\beta=\frac{2(\theta-1)}{2 \theta-1}, \quad \theta=\frac{1-\frac{\beta}{2}}{1-\beta}, \quad 0<\beta<\frac{11}{20}, \quad 1<\theta<\frac{29}{18},
$$

and define

$$
c_{0}=-\frac{\int Q}{2}(\theta-1)(2 \theta-1)^{\theta-1} .
$$

Given $s>s_{0},(b(s), \lambda(s), x(s)) \in \mathbb{R}_{+}^{*} \times \mathbb{R}_{+}^{*} \times \mathbb{R}$, we define:

$$
g(s)=\frac{b(s)}{\lambda^{2}(s)}+\frac{4}{\int Q} c_{0} \lambda^{-\frac{3}{2}}(s) x^{-\theta}(s), \quad f(s)=\lambda^{\frac{1}{2}}(s)+\frac{2}{\int Q} c_{0} \frac{1}{\theta-1} x^{-\theta+1}(s) .
$$

Let $\left(\varphi_{i}\right)_{i=1,2}, \psi$ be smooth functions such that:

$$
\begin{aligned}
& \varphi_{i}(y)= \begin{cases}e^{y} & \text { for } y<-1, \\
1+y & \text { for }-\frac{1}{2}<y<\frac{1}{2}, \quad \varphi_{i}^{\prime}(y)>0, \quad \forall y \in \mathbb{R}, \\
y^{i} & \text { for } y>2,\end{cases} \\
& \psi(y)=\left\{\begin{array}{ll}
e^{2 y} & \text { for } y<-1, \\
1 & \text { for } y>-\frac{1}{2},
\end{array} \psi^{\prime}(y) \geq 0 \forall y \in \mathbb{R} .\right.
\end{aligned}
$$

Let $B>100$ and

$$
\psi_{B}(y)=\psi\left(\frac{y}{B}\right), \varphi_{i, B}=\varphi_{i}\left(\frac{y}{B}\right), \quad i=1,2,
$$

and define the following norms on $\varepsilon$

$$
\begin{aligned}
\mathcal{N}_{i}(s) & =\int \varepsilon_{y}^{2}(s, y) \psi_{B}(y) d y+\int \varepsilon^{2}(s, y) \varphi_{i, B}(y) d y, \\
\mathcal{N}_{i, \text { loc }}(s) & =\int \varepsilon^{2}(s, y) \varphi_{i, B}^{\prime}(y) d y, \quad i=1,2 .
\end{aligned}
$$


We now claim the following bootstrap proposition which is the heart of the analysis:

Proposition 3.1 (Bootstrap). Let $s_{0}=s_{0}(\beta)>1$ large enough and set

$$
x_{0}=x\left(s_{0}\right)=\frac{1}{(1-\beta)} s_{0}^{1-\beta} .
$$

Let $\varepsilon_{0} \in H^{1}$ be such that

$$
s_{0}^{10}\left[\int_{y>0} y^{10} \varepsilon_{0}^{2}(y) d y+\left\|\varepsilon_{0}\right\|_{H^{1}}^{2}\right]<1,\left(\varepsilon_{0}, y \Lambda Q\right)=\left(\varepsilon_{0}, \Lambda Q\right)=\left(\varepsilon_{0}, Q\right)=0 .
$$

Then, there exists

$$
\left(\lambda_{0}, b_{0}\right) \in \mathcal{D}=\left\{(\lambda, b):\left|\lambda-s_{0}^{-\beta}\right| \leq s_{0}^{-\beta-\frac{1}{10}},\left|b-\beta s_{0}^{-1}\right| \leq s_{0}^{-1-\frac{1}{10}}\right\},
$$

such that the solution of (1.1) with initial data

$$
u_{0}(x)=\frac{1}{\lambda_{0}^{\frac{1}{2}}}\left(Q_{b_{0}}+\lambda_{0}^{\frac{1}{2}} q_{0}\left(s_{0}, x_{0}\right) Y_{0}+\varepsilon_{0}\right)\left(\frac{x-x_{0}}{\lambda_{0}}\right)+q_{0}\left(s_{0}, x\right)
$$

has a decomposition $(b(s), \lambda(s), x(s), \varepsilon(s))$ as in Proposition 2.4 which satisfies ${ }^{2}$ on $\left[s_{0},+\infty\right)$ :

(BS1) $\quad\left(|g(s)| s^{1-2 \beta+\frac{1}{5}}\right)^{2}+\left(|f(s)| s^{\frac{\beta}{2}+\frac{1}{10}}\right)^{2} \leq 1$;

(BS2) $|b(s)| \leq 10 s^{-1}, \frac{1}{10} s^{-\beta} \leq \lambda(s) \leq 10 s^{-\beta}, \frac{1}{10} s^{1-\beta} \leq(1-\beta) x(s) \leq 10 s^{1-\beta}$;

(BS3) $\quad \int_{y>0} y^{10} \varepsilon^{2}(s, y) d y \leq 10 \lambda^{-10}, \quad \mathcal{N}_{i}(s) \leq s^{-\frac{5}{2}}, \quad\|\varepsilon(s)\|_{H^{1}} \lesssim \delta\left(\alpha^{*}\right)$.

Moreover,

$$
\begin{gathered}
\left|\frac{(1-\beta)}{s^{1-\beta}} x(s)-1\right|+\left|s^{\beta} \lambda(s)-1\right|+\left|\frac{s}{\beta} b(s)-1\right| \lesssim s^{-\frac{1}{10}}, \\
\lambda^{-1}\left\|\varepsilon_{y}(s)\right\|_{L^{2}}+\|\varepsilon(s)\|_{L^{2}} \lesssim 1
\end{gathered}
$$

${ }^{2}$ Recall that $s=s(t)$ is the rescaled time (2.30). 
Let us observe that (3.10) now gives the leading order behavior of the scaling parameter $\lambda(s)=\frac{1}{s^{\beta}}(1+o(1))$, and the conclusion of Theorem 1.1 now immediately follow from the change of variables (2.30) depending on the value of $\beta$ as in step (iv) of Section 1.4.

The rest of this section is therefore devoted to the proof of Proposition 3.1. First observe by uniqueness of the decomposition that

$$
b_{0}=b\left(s_{0}\right), \quad \lambda\left(s_{0}\right)=\lambda_{0} \quad x\left(s_{0}\right)=x_{0}, \quad \varepsilon\left(s_{0}\right)=\varepsilon_{0} .
$$

We now argue by contradiction, assuming that for all $\left(\lambda_{0}, b_{0}\right) \in \mathcal{D}$, we have $s^{*}\left(\lambda_{0}, b_{0}\right):=\sup \left\{s \geq s_{0}\right.$ such that (BS1)-(BS2)-(BS3) holds on $\left.\left[s_{0}, s\right]\right\}<+\infty$.

We will derive a contradiction by first closing the bootstrap bounds (BS1)-(BS2)(BS3), and then finding a pair $\left(\lambda_{0}, b_{0}\right)$ using a topological argument.

\subsection{First consequences of the bootstrap bounds}

Let us start with some quantitative bounds which follow from the bootstrap bounds and Proposition 2.4.

Claim 3.2 (Consequences of the bootstrap estimates). (i) For $s_{0}=s_{0}(\beta)$ large enough, there holds:

- if $\beta>\frac{1}{3}$, for all $s \in\left(s_{0}, s^{*}\right), t(s)=\int_{s_{0}}^{s} \lambda^{3}\left(s^{\prime}\right) d s^{\prime}<1$;

- if $0<\beta \leq \frac{1}{3}$, for all $t>0, x(t) \geq \frac{2}{3} t+\frac{2}{3} x_{0}$.

(ii) For all $s \in\left(s_{0}, s^{*}\right)$,

$$
\begin{aligned}
& 0<-p(s) \lesssim \frac{1}{s}, \quad \frac{\lambda}{x} \lesssim \frac{1}{s} \\
&\left|\frac{\lambda_{s}}{\lambda}+b\right|+\left|\frac{x_{s}}{\lambda}-1\right| \lesssim\left(\int \varepsilon^{2} e^{\left.-\frac{|y|}{10}\right)^{\frac{1}{2}}+\frac{1}{s^{2}}}\right. \\
&\left|\frac{\lambda_{s}}{\lambda}\right| \lesssim \frac{1}{s}+\left(\int \varepsilon^{2} e^{-\frac{|y|}{10}}\right)^{\frac{1}{2}}, \quad\left|x_{s}\right| \lesssim s^{-\beta}\left(1+\left(\int \varepsilon^{2} e^{-\frac{|y|}{10}}\right)^{\frac{1}{2}}\right) \\
&\left|b_{s}\right|+\left|p_{s}\right| \lesssim \int \varepsilon^{2} e^{-\frac{|y|}{10}}+\frac{1}{s^{2}}, \\
&\left|\frac{d}{d s}\left(\frac{b}{\lambda^{2}}+\frac{4}{\left(\int Q\right)} c_{0} \lambda^{-\frac{3}{2}}(s) x^{-\theta}(s)\right)\right| \lesssim \frac{1}{\lambda^{2}}\left(\frac{1}{s^{\frac{29}{10}}}+\frac{1}{s}\left(\int \varepsilon^{2} e^{-\frac{|y|}{10}}\right)^{\frac{1}{2}}+\int \varepsilon^{2} e^{-\frac{|y|}{10}}\right) .
\end{aligned}
$$

(iii) For all $s \in\left(s_{0}, s^{*}\right)$,

$$
\begin{aligned}
\|\varepsilon(s)\|_{L^{2}}^{2} & \lesssim\left|\int u^{2}(0)-\int Q^{2}\right|+s^{-1}+x_{0}^{-\theta+\frac{1}{2}}, \\
\frac{1}{\lambda^{2}}\left\|\varepsilon_{y}(s)\right\|_{L^{2}}^{2} & \lesssim|E(u(0))|+s^{-\frac{1}{10}}+x_{0}^{-\theta-\frac{1}{2}} .
\end{aligned}
$$


Proof. Let $\frac{1}{3}<\beta<\frac{11}{20}$. Then

$$
t(s)=\int_{s_{0}}^{s} \lambda^{3}\left(s^{\prime}\right) d s^{\prime} \leq 10^{3} \int_{s_{0}}^{s}\left(s^{\prime}\right)^{-3 \beta} d s^{\prime} \leq \frac{10^{3}}{3 \beta-1}\left(s_{0}^{-3 \beta+1}-s^{-3 \beta+1}\right)<1
$$

for $s_{0}$ large enough. For $\beta=\frac{1}{3}$,

$$
t(s)=\int_{s_{0}}^{s} \lambda^{3}\left(s^{\prime}\right) d s^{\prime} \leq 10^{3} \log \frac{s}{s_{0}} \quad \text { so that } \quad s \geq s_{0} e^{10^{-3} t} .
$$

Thus

$$
x(s) \geq \frac{3}{20} s^{\frac{2}{3}} \geq \frac{3}{20} s_{0}^{\frac{2}{3}} e^{\frac{2}{3} 10^{-3} t} \geq 10 t^{3}
$$

for $s_{0}$ large enough. Since $x(s) \geq \frac{4}{5} x_{0}$, we obtain $x(t)>t^{3}+\frac{1}{2} x_{0}$. Finally, for $0<\beta<\frac{1}{3}$,

$$
t(s)=\int_{s_{0}}^{s} \lambda^{3}\left(s^{\prime}\right) d s^{\prime} \leq \frac{10^{3}}{1-3 \beta}\left(s^{1-3 \beta}-s_{0}^{1-3 \beta}\right)
$$

so that for large $s_{0}$

$$
s \geq\left(\frac{(1-3 \beta) t}{10^{3}}+s_{0}^{1-3 \beta}\right)^{\frac{1}{1-3 \beta}} \geq 100 t^{\frac{1+\beta}{1-\beta}},
$$

and

$$
x(s) \geq \frac{3}{20} s^{1-\beta} \geq 15 t^{1+\beta} .
$$

Since $x(s) \geq \frac{4}{5} x_{0}$, we obtain $x(t)>t^{1+\beta}+\frac{2}{3} x_{0}$.

The estimate (3.12) is a consequence of (2.41) and $\frac{\beta}{2}+(1-\beta) \theta=1$, so that

$$
0<p \lesssim \lambda^{\frac{1}{2}} x^{-\theta}(s) \lesssim s^{-\frac{\beta}{2}} s^{-\theta(1-\beta)} \lesssim \frac{1}{S} .
$$

The estimates (3.13)-(3.16) are immediate consequences of (2.31)-(2.33), (2.45), the bootstrap assumptions and the upper bound $\beta<\frac{11}{20}$.

\subsection{Closing the estimates on $\varepsilon$}

We now close the bounds on $\varepsilon$ and claim the improved bound: for all $s \in\left[s_{0}, s^{*}\right]$,

$\left(\mathrm{BS3}^{\prime}\right) \quad \int_{y>0} y^{10} \varepsilon^{2}(s, x) d x \leq 5 \lambda^{-10}, \quad \mathcal{N}_{i}(s) \leq \frac{1}{2} s^{-\frac{5}{2}}, \quad\|\varepsilon(s)\|_{H^{1}} \lesssim \delta\left(\alpha^{*}\right)$.

Let $\varphi_{10}$ be a smooth function such that

$$
\varphi_{10}(y)=\left\{\begin{array}{l}
0 \text { for } y \leq 0, \\
y^{10} \text { for } y \geq 1
\end{array}, \quad 0 \leq \varphi_{10} \lesssim \varphi_{10}^{\prime} \text { for } 0<y<1 .\right.
$$

The control of the tail of $\varepsilon$ on the right is a direct consequence of the following brute force monotoncity formula: 
Lemma 3.3 (Dynamical control of the tail on the right). For all $s \in\left[s_{0}, s^{*}\right]$,

$$
\lambda^{-10} \frac{d}{d s}\left\{\lambda^{10} \int \varphi_{10} \varepsilon^{2}\right\} \lesssim \mathcal{N}_{1, \text { loc }}+\frac{1}{s^{2}} .
$$

See proof of Lemma 3.3 in Appendix B.

The control of the $\mathcal{N}_{i}(\varepsilon)$ norm, which is fundamental for the proof, now follows by adapting the mixed Energy/Morawetz monotonicity formula first derived in [38]. Recalling the definitions (3.2), (3.3), we claim:

Lemma 3.4 (Monotonicity formula). There exist $\mu>0$ such that the following holds for $B>100$ large enough. Let the energy-virial Lyapounov functionals for $i=1,2$,

$$
\begin{aligned}
\mathcal{F}_{i}=\int[ & \varepsilon_{y}^{2} \psi_{B}+\varepsilon^{2} \varphi_{i, B}-\frac{1}{3}\left(\left(\varepsilon+Q_{b}+p Y_{0}+q\right)^{6}-\left(Q_{b}+p Y_{0}+q\right)^{6}\right. \\
& \left.\left.-6 \varepsilon\left(Q_{b}^{5}+q^{5}+5 Q^{4}\left(p Y_{0}+q\right)\right)\right) \psi_{B}\right] .
\end{aligned}
$$

Then the following estimates hold on $\left[s_{0}, s^{*}\right]$ :

(i) Lyapounov control: for $i=1,2, j \geq 0$

$$
\frac{d}{d s}\left[s^{j} \mathcal{F}_{i}\right]+\mu s^{j} \int\left(\varepsilon_{y}^{2}+\varepsilon^{2}\right) \varphi_{i, B}^{\prime} \lesssim s^{j-4}+s^{j-9+10 \beta} .
$$

(iii) Coercivity of $\mathcal{F}_{i}$ and pointwise bounds: for $i=1,2, j \geq 0$,

$$
-\frac{1}{s^{4}}+\mathcal{N}_{i} \lesssim \mathcal{F}_{i} \lesssim \frac{1}{s^{4}}+\mathcal{N}_{i}
$$

See the proof of Lemma 3.4 in Appendix B.

Proof of $\left(\mathrm{BS}^{\prime}\right)$. From Lemma 3.3, (BS2) and (BS3),

$$
\frac{d}{d s}\left\{\lambda^{10} \int \varphi_{10} \varepsilon^{2}\right\} \lesssim \lambda^{10}\left(\mathcal{N}_{1, \text { loc }}+s^{-2}\right) \lesssim s^{-10 \beta-2},
$$

so that by integration on $\left[s_{0}, s\right]$, and (3.7),

$$
\lambda^{10}(s) \int \varphi_{10} \varepsilon^{2}(s) \leq \lambda^{10}\left(s_{0}\right) \int \varphi_{10} \varepsilon^{2}\left(s_{0}\right)+C s_{0}^{-1-10 \beta} \leq 2 .
$$

By the properties of $\varphi_{10}$ and (3.7), we obtain

$$
\lambda^{10}(s) \int_{y>0} y^{10} \varepsilon^{2}(s, y) d y \leq 3 .
$$


Now, we apply Lemma 3.4 with $j=\frac{5}{2}$. We find by (3.21) and $\beta<\frac{11}{20}$,

$$
\frac{d}{d s}\left[s^{\frac{5}{2}} \mathcal{F}_{i}\right] \lesssim s^{-\frac{3}{2}}+s^{-1-10\left(\frac{11}{20}-\beta\right)}
$$

The initial smallness (3.7) ensures $s_{0}^{10}\left|\mathcal{F}_{i}(0)\right| \lesssim 1$ and thus the time integration of (3.24) on $\left[s_{0}, s^{*}\right]$ yields $\mathcal{F}_{i}(s) \lesssim s^{-\frac{5}{2}} s_{0}^{-\delta}$ for some $\delta=\delta(\beta)>0$. Using (3.22), we conclude $\mathcal{N}_{i}(s) \leq s^{-\frac{5}{2}} s_{0}^{-\delta}+s^{-4} \leq \frac{1}{2} s^{-\frac{5}{2}}$ for $s_{0}$ large enough, which together with (3.23) and the control of the full $H^{1}$ norm through the conservation laws (2.27), (2.28) concludes the proof of $\left(\mathrm{BS}^{\prime}\right)$.

\subsection{Closing the estimates on $(b, \lambda, x)$}

We now use the obtained bounds on $\varepsilon$ and the modulation equations on the geometrical parameters of Proposition 2.4 to close the bounds on $(b, \lambda, x)$. We claim: for all $s \in\left[s_{0}, s^{*}\right]$,

$\left(\mathrm{BS} 2^{\prime}\right) \quad|b(s)| \leq 5 s^{-1}, \frac{1}{5} s^{-\beta} \leq \lambda(s) \leq 5 s^{-\beta}, \frac{1}{5} s^{1-\beta} \leq(1-\beta) x(s) \leq 5 s^{1-\beta}$.

Proof of $\left(\mathrm{BS}^{\prime}\right)$. First, note that from (2.33), (2.31), and using (BS2)-(BS3), $\frac{1}{3} \leq$ $\beta \leq \frac{9}{10}$, we have on $\left[s_{0}, s^{*}\right]$ :

$$
\begin{aligned}
& \left|g_{s}\right| \lesssim s^{-\frac{9}{4}+2 \beta}, \\
& \left|\frac{\lambda_{s}}{\lambda}+b\right| \lesssim s^{-\frac{5}{4}}, \\
& \left|\frac{x_{s}}{\lambda}-1\right| \lesssim s^{-\frac{5}{4}} .
\end{aligned}
$$

By $(\mathrm{BS} 1)$ and $(\mathrm{BS} 2)$, we have using $(1-\beta)(\theta-1)=\frac{\beta}{2}$ the estimate:

$$
\begin{aligned}
& \left|\lambda(s)-\left(\frac{2}{\int Q} c_{0} \frac{1}{\theta-1}\right)^{2} x^{-2 \theta+2}(s)\right| \\
& =\left|\lambda^{\frac{1}{2}}(s)+\frac{2}{\int Q} c_{0} \frac{1}{\theta-1} x^{-\theta+1}(s)\right|\left|\lambda^{\frac{1}{2}}(s)-\frac{2}{\int Q} c_{0} \frac{1}{\theta-1} x^{-\theta+1}(s)\right| \\
& \lesssim|f(s)|\left(\lambda^{\frac{1}{2}}(s)+x^{-\theta+1}(s)\right) \lesssim s^{-\frac{\beta}{2}-\frac{1}{10}}\left(s^{-\frac{\beta}{2}}+s^{-(1-\beta)(\theta-1)}\right) \lesssim s^{-\beta-\frac{1}{10}}
\end{aligned}
$$

Using (3.27), we find

$$
\left|x_{s}(s)-\left(\frac{2}{\int Q} c_{0} \frac{1}{\theta-1}\right)^{2} x^{-2 \theta+2}(s)\right| \lesssim s^{-\beta-\frac{1}{10}}+s^{-\frac{5}{4}-\beta} \lesssim s^{-\beta-\frac{1}{10}}
$$


and hence

$$
\left|\left(x^{\frac{1}{1-\beta}}\right)_{s}-(1-\beta)^{-(1-\beta)}\right| \lesssim s^{-\frac{1}{10}}
$$

from $2 \theta-1=\frac{1}{1-\beta}$ and the choice of $c_{0}$ in (3.1) which gives:

$$
(1-\beta)^{-(1-\beta)}=(2 \theta-1)\left(\frac{2}{\int Q} c_{0} \frac{1}{\theta-1}\right)^{2} .
$$

Since from (3.6) we have $\frac{(1-\beta)}{s_{0}^{1-\beta}} x\left(s_{0}\right)=1$, the time integration of (3.29) on $\left[s_{0}, s\right]$ yields $\left|x^{\frac{1}{\beta-1}}(s)-(1-\beta)^{-\frac{1}{\beta-1}} s\right| \lesssim s^{1-\frac{1}{10}}$. Thus,

$$
\left|\frac{(1-\beta)}{s^{1-\beta}} x(s)-1\right| \lesssim s^{-\frac{1}{10}} .
$$

Inserting (3.30) into (3.28), we find for $\lambda$,

$$
\left|s^{\beta} \lambda(s)-1\right| \lesssim s^{-\frac{1}{10}}
$$

Finally, using $|g(s)| \leq s^{-1+2 \beta-\frac{1}{5}}$, we find

$$
\left|\frac{s}{\beta} b(s)-1\right| \lesssim s^{-\frac{1}{10}} .
$$

From (3.30), (3.31) and (3.32), (BS2') follows for $s_{0}$ large enough.

\subsection{Choice of $\lambda_{0}$ and $b_{0}$ by a topological argument}

We now claim from a standard topological argument based on the outgoing behavior of the ODE's for $(f, g)$ that we can find $\left(b_{0}, \lambda_{0}\right) \in \mathcal{D}$ such that the remaining condition (BS1) is closed. Indeed, let

$$
G(s)=g(s) s^{1-2 \beta+\frac{1}{5}}, \quad F(s)=f(s) s^{\frac{\beta}{2}+\frac{1}{10}},
$$

and

$$
H(s)=F^{2}(s)+G^{2}(s) .
$$

From $\left(\mathrm{BS}^{\prime}\right)$ and $\left(\mathrm{BS}^{\prime}\right)$, since $s^{*}=s^{*}\left(x_{0}, b_{0}\right)<+\infty$, it follows from a standard continuity argument that at $s=s^{*} \geq s_{0}$,

$$
H\left(s^{*}\right)=1 \text {. }
$$

We first claim the strict outgoing behavior:

$$
H^{\prime}\left(s^{*}\right) \geq \frac{1}{20 s^{*}} .
$$


Proof of (3.34). Since

$$
G^{\prime}(s)=\left(1-2 \beta+\frac{1}{5}\right) g(s) s^{-2 \beta+\frac{1}{5}}+g^{\prime}(s) s^{1-2 \beta+\frac{1}{5}},
$$

we have using (3.25):

$$
G^{\prime}\left(s^{*}\right)=\left(1-2 \beta+\frac{1}{5}\right) \frac{G\left(s^{*}\right)}{s^{*}}+O\left(\left(s^{*}\right)^{-\left(1+\frac{1}{20}\right)}\right) .
$$

Similarly,

$$
F^{\prime}\left(s^{*}\right)=\left(\frac{\beta}{2}+\frac{1}{10}\right) f\left(s^{*}\right)\left(s^{*}\right)^{\frac{\beta}{2}+\frac{1}{10}-1}+f^{\prime}\left(s^{*}\right)\left(s^{*}\right)^{\frac{\beta}{2}+\frac{1}{10}} .
$$

We now estimate $f^{\prime}(s)$. By direct computations and then (3.26), (3.27) and (BS1)(BS2),

$$
\begin{aligned}
f^{\prime}(s) & =\frac{1}{2} \frac{\lambda_{s}}{\lambda} \lambda^{\frac{1}{2}}-\frac{2}{\int Q} c_{0} x_{s} x^{-\theta} \\
& =\frac{1}{2} \lambda^{\frac{5}{2}}\left[\frac{b}{\lambda^{2}}-\frac{4}{\int Q} c_{0} \lambda^{-\frac{3}{2}} x^{-\theta}\right]+O\left(s^{-\frac{5}{4}-\frac{\beta}{2}}\right) \\
& =\frac{1}{2} \lambda^{\frac{5}{2}} g(s)+O\left(s^{-\frac{5}{4}-\frac{\beta}{2}}\right)=O\left(s^{-1-\frac{\beta}{2}-\frac{1}{5}}\right) .
\end{aligned}
$$

Thus,

$$
F^{\prime}\left(s^{*}\right)=\left(\frac{\beta}{2}+\frac{1}{10}\right) \frac{F\left(s^{*}\right)}{s^{*}}+O\left(\left(s^{*}\right)^{-1-\frac{1}{10}}\right) .
$$

Therefore

$$
H^{\prime}\left(s^{*}\right)=2 F^{\prime}\left(s^{*}\right) F\left(s^{*}\right)+2 G^{\prime}\left(s^{*}\right) G\left(s^{*}\right) \geq \frac{1}{10} \frac{H\left(s^{*}\right)}{s^{*}}+O\left(\left(s^{*}\right)^{-1-\frac{1}{20}}\right) \geq \frac{1}{20 s^{*}},
$$

for $s_{0}$ large enough.

By standard arguments (see, e.g., the proof of Lemma 6 in [4]), the strict outgoing behavior (3.34) ensures that the map $\left(\lambda_{0}, b_{0}\right) \in \mathcal{D} \rightarrow s^{*}\left(x_{0}, b_{0}\right)$ is continuous. We define the continuous maps

$$
\begin{aligned}
\lambda_{0}\left(F_{0}\right) & =s_{0}^{-\beta}\left(1-F_{0} s_{0}^{-\frac{1}{10}}\right)^{2} \\
b_{0}\left(F_{0}, G_{0}\right) & =\beta s_{0}^{-1+\frac{\beta}{2}} \lambda_{0}^{\frac{1}{2}}\left(F_{0}\right)\left(1+\frac{1}{\beta} G_{0} s_{0}^{\frac{3}{2} \beta-\frac{1}{5}} \lambda_{0}^{\frac{3}{2}}\left(F_{0}\right)\right) \\
& =\beta s_{0}^{-1}\left|1-F_{0} s_{0}^{-\frac{1}{10}}\right|\left(1+\frac{1}{\beta} G_{0} s_{0}^{-\frac{1}{5}}\left|1-F_{0} s_{0}^{-\frac{1}{10}}\right|^{3}\right)
\end{aligned}
$$


so that

$$
\begin{aligned}
G_{0} & =s_{0}^{1-2 \beta+\frac{1}{5}}\left(\frac{b_{0}}{\lambda_{0}^{2}}-\frac{4}{\int Q} c_{0} \lambda_{0}^{-\frac{3}{2}} x_{0}^{-\theta}\right) \\
F_{0} & =s_{0}^{\frac{\beta}{2}+\frac{1}{10}}\left(\lambda_{0}^{\frac{1}{2}}+\frac{2}{\int Q} c_{0} \frac{1}{\theta-1} x_{0}^{-\theta+1}\right) .
\end{aligned}
$$

Now, consider the continous map

$$
\begin{aligned}
\mathcal{M}: \quad \mathcal{B}_{\mathbb{R}^{2}} & \rightarrow \mathcal{S}_{\mathbb{R}^{2}} \\
\left(F_{0}, G_{0}\right) & \mapsto\left(F\left(s^{*}\left(\lambda_{0}\left(F_{0}\right), b_{0}\left(F_{0}, G_{0}\right)\right)\right), G\left(s^{*}\left(\lambda_{0}\left(F_{0}\right), b_{0}\left(F_{0}, G_{0}\right)\right)\right)\right) .
\end{aligned}
$$

where $\mathcal{B}_{\mathbb{R}^{2}}$ and $\mathcal{S}_{\mathbb{R}^{2}}$ are, respectively, the ball and the sphere of $\mathbb{R}^{2}$ of radius 1 . For $\left(F_{0}, G_{0}\right) \in \mathcal{S}_{\mathbb{R}^{2}}$, we have $\mathcal{M}\left(F_{0}, G_{0}\right)=\left(F_{0}, G_{0}\right)$, in other words, $\mathcal{M}$ is the identity on the sphere $\mathcal{S}_{\mathbb{R}^{2}}$. The existence of such a continuous map $\mathcal{M}$ is in contradiction with Brouwer's fixed point theorem. Therefore, there exists $\lambda_{0}$ and $b_{0}$ such that

$$
\left|\lambda_{0}-s_{0}^{-\beta}\right| \leq s_{0}^{-\beta-\frac{1}{10}}, \quad\left|b_{0}-\beta s_{0}^{-1}\right| \leq s_{0}^{-1-\frac{1}{10}},
$$

and $s^{*}\left(\lambda_{0}, b_{0}\right)=+\infty$. In particular (BS1)-(BS2)-(BS3) hold on $\left[s_{0},+\infty\right)$. Finally, (3.30), (3.31) and (3.32) imply (3.10).

This concludes the proof of Proposition 3.1 and therefore also of Theorem 1.1.

\section{A. Proof of Lemma 2.3}

Recall that $c_{0} \in \mathbb{R}$ and $\theta>1$ are fixed, $x_{0} \gg 1$ is to be taken large enough and $q_{0}$ is the solution of

$$
\partial_{t} q_{0}+\partial_{x}\left(\partial_{x}^{2} q_{0}+q_{0}^{5}\right)=0, \quad q_{0}(0, x)=f_{0}(x)
$$

where the function $f_{0}$ is smooth and satisfies

$$
\begin{aligned}
& f_{0}(x)=c_{0} x^{-\theta} \text { for } x>\frac{x_{0}}{2}, \quad f_{0}(x)=0 \text { for } x<\frac{x_{0}}{4}, \\
& \text { for all } x \in \mathbb{R} \text {, for all } k \geq 0, \quad\left|\frac{d^{k} f_{0}}{d x^{k}}(x)\right| \lesssim c_{0}|x|^{-\theta-k} \text {. }
\end{aligned}
$$

First, for $x_{0}$ large enough, $\left\|f_{0}\right\|_{L^{2}}$ is small and it follows from the $L^{2}$ and $H^{s}$ Cauchy theory (Corollary 2.9 in [20]) that $q_{0}$ is global and bounded in $H^{s}$ for all $s \geq 0$, with

$$
\sup _{t}\left\|q_{0}(t)\right\|_{H^{s}} \lesssim \delta\left(x_{0}^{-1}\right)
$$


We define

$$
\begin{gathered}
q_{1}(t, x)=q_{0}(t, x)-f_{0}(x), \\
\partial_{t} q_{1}+\partial_{x}\left(\partial_{x}^{2} q_{1}+\left(q_{1}+f_{0}\right)^{5}-f_{0}^{5}\right)=F_{0}, \quad q_{1}(0, x)=0,
\end{gathered}
$$

where $F_{0}=-\partial_{x}^{3} f_{0}-\partial_{x}\left(f_{0}^{5}\right)$.

For any $\bar{\theta} \geq 0$, define a smooth function $\varphi_{\bar{\theta}}$ such that

$$
\bar{\theta}(x)=x^{\bar{\theta}} \text { for } x \geq 4, \quad \varphi_{\bar{\theta}}(x)=e^{\frac{x}{8}} \text { for } x \leq 0, \quad \varphi^{\prime} \geq 0, \varphi^{\prime \prime \prime} \leq \frac{1}{4} \varphi^{\prime} \text { on } \mathbb{R} .
$$

For $0 \leq \theta_{1}<2 \theta+4, \quad \theta_{1} \neq 2 \theta+3$, set

$$
\begin{aligned}
M_{\theta_{1}}(t) & =\int q_{1}^{2}(t) \varphi_{\theta_{1}}\left(x-\frac{t}{4}-\frac{x_{0}}{4}\right) d x, \\
E_{\theta_{1}}(t) & =\int\left(\left(\partial_{x} q_{1}\right)^{2}(t)-\frac{1}{3}\left(\left(q_{1}+f_{0}\right)^{6}-f_{0}^{6}-6 q_{1} f_{0}^{5}\right)\right) \varphi_{\theta_{1}+2}\left(x-\frac{t}{4}-\frac{x_{0}}{4}\right) d x, \\
F_{\theta_{1}, k}(t) & =\int\left(\partial_{x}^{k} q_{1}\right)^{2}(t) \varphi_{\theta_{1}+2 k}\left(x-\frac{t}{4}-\frac{x_{0}}{4}\right) d x, k \geq 2 .
\end{aligned}
$$

We differentiate $M_{\theta_{1}}(t)$ (omitting the variable $x-\frac{t}{4}-\frac{x_{0}}{4}$ for the function $\varphi_{\theta_{1}}$ ):

$$
\begin{aligned}
M_{\theta_{1}}^{\prime}(t)= & -3 \int\left(\partial_{x} q_{1}\right)^{2} \varphi_{\theta_{1}}^{\prime}-\frac{1}{4} \int q_{1}^{2} \varphi_{\theta_{1}}^{\prime}+\int q_{1}^{2} \varphi_{\theta_{1}}^{\prime \prime \prime} \\
& +2 \int\left(\left(q_{1}+f_{0}\right)^{5}-f_{0}^{5}\right)\left(q_{1} \varphi_{\theta_{1}}\right)_{x}+2\left|\int F_{0} q_{1} \varphi_{\theta_{1}}\right| \\
\leq & -3 \int\left(\partial_{x} q_{1}\right)^{2} \varphi_{\theta_{1}}^{\prime}-\frac{3}{16} \int q_{1}^{2} \varphi_{\theta_{1}}^{\prime} \\
& -2 \int\left[\frac{\left(q_{1}+f_{0}\right)^{6}}{6}-\frac{f_{0}^{6}}{6}-\left(q_{1}+f_{0}\right)^{5} q_{1}\right] \varphi_{\theta_{1}}^{\prime} \\
& +2 \int\left[\left(q_{1}+f_{0}\right)^{5}-5 q_{1}^{4} f_{0}-f_{0}^{5}\right] f_{0}^{\prime} \varphi_{\theta_{1}}+2\left|\int F_{0}^{2} \frac{\varphi_{\theta_{1}}^{2}}{\varphi_{\theta_{1}}^{\prime}}\right|^{\frac{1}{2}}\left|\int q_{1}^{2} \varphi_{\theta_{1}}^{\prime}\right|^{\frac{1}{2}} \\
\leq & -3 \int\left(\partial_{x} q_{1}\right)^{2} \varphi_{\theta_{1}}^{\prime}-\frac{1}{8} \int q_{1}^{2} \varphi_{\theta_{1}}^{\prime}+\delta\left(x_{0}^{-1}\right) \int q_{1}^{2} \varphi_{\theta_{1}}^{\prime}+C \int F_{0}^{2} \frac{\varphi_{\theta_{1}}^{2}}{\varphi_{\theta_{1}}^{\prime}} .
\end{aligned}
$$

Thus, for $x_{0}$ large enough, we have obtained

$$
M_{\theta_{1}}^{\prime}(t)+\frac{1}{10} \int\left[\left(\partial_{x} q_{1}\right)^{2}+q_{1}^{2}\right] \varphi_{\theta_{1}}^{\prime} \lesssim \int F_{0}^{2} \frac{\varphi_{\theta_{1}}^{2}}{\varphi_{\theta_{1}}^{\prime}}
$$


For $x_{0}$ large enough,

$$
\begin{aligned}
\int F_{0}^{2} \frac{\varphi_{\theta_{1}}^{2}}{\varphi_{\theta_{1}}^{\prime}}\left(x-\frac{t}{4}-\frac{x_{0}}{4}\right) \lesssim & \int_{x>\frac{t}{4}+\frac{x_{0}}{4}+4} x^{-2(\theta+3)}\left(x-\frac{t}{4}-\frac{x_{0}}{4}\right)^{\theta_{1}+1} \\
& +\int_{\frac{t}{8}+\frac{x_{0}}{8}<x<\frac{t}{4}+\frac{x_{0}}{4}+4} x^{-2(\theta+3)} \\
& +\int_{x<\frac{t}{8}+\frac{x_{0}}{8}} e^{\frac{1}{8}\left(x-\frac{t}{4}-\frac{x_{0}}{4}\right)} \\
\lesssim & \left(t+x_{0}\right)^{\theta_{1}-2 \theta-4}
\end{aligned}
$$

Therefore, using also $M_{\theta_{1}}(0)=0$, we find by integration:

$$
M_{\theta_{1}}(t)+\int_{0}^{t} \int\left[\left(\partial_{x} q_{1}\right)^{2}+q_{1}^{2}\right] \varphi_{\theta_{1}}^{\prime} \lesssim \begin{cases}\left(t+x_{0}\right)^{\theta_{1}-2 \theta-3} & \text { if } 0<\theta_{1}-2 \theta-3<1 \\ x_{0}^{\theta_{1}-2 \theta-3} & \text { if } \theta_{1}-2 \theta-3<0 .\end{cases}
$$

We argue similarly for $E_{\theta_{1}}(t)$.

$$
\begin{aligned}
E_{\theta_{1}}^{\prime}(t)= & 2 \int \partial_{t} q_{1}\left[-\partial_{x}^{2} q_{1}-\left(\left(q_{1}+f_{0}\right)^{5}-f_{0}^{5}\right)\right] \varphi_{\theta_{1}+2}-2 \int \partial_{t} q_{1} \partial_{x} q_{1} \varphi_{\theta_{1}+2}^{\prime} \\
& -\frac{1}{4} \int\left[\left(\partial_{x} q_{1}\right)^{2}(t)-\frac{1}{3}\left(\left(q_{1}+f_{0}\right)^{6}-f_{0}^{6}-6 q_{1} f_{0}^{5}\right)\right] \varphi_{\theta_{1}+2}^{\prime} \\
= & -\int\left[\partial_{x}^{2} q_{1}+\left(\left(q_{1}+f_{0}\right)^{5}-f_{0}^{5}\right)\right]^{2} \varphi_{\theta_{1}+2}^{\prime} \\
& -2 \int\left[\partial_{x}^{2} q_{1}+\left(q_{1}+f_{0}\right)^{5}-f_{0}^{5}\right] F_{0} \varphi_{\theta_{1}+2} \\
& +2 \int\left[\partial_{x}^{2} q_{1}+\left(q_{1}+f_{0}\right)^{5}-f_{0}^{5}\right] \partial_{x} q_{1} \varphi_{\theta_{1}+2}^{\prime}-2 \int F_{0} \partial_{x} q_{1} \varphi_{\theta_{1}+2}^{\prime} \\
& -\frac{1}{4} \int\left[\left(\partial_{x} q_{1}\right)^{2}(t)-\frac{1}{3}\left(\left(q_{1}+f_{0}\right)^{6}-f_{0}^{6}-6 q_{1} f_{0}^{5}\right)\right] \varphi_{\theta_{1}+2}^{\prime} .
\end{aligned}
$$

We use the following computations and estimates

$$
\begin{aligned}
& \left|\int\left[\partial_{x}^{2} q_{1}+\left(q_{1}+f_{0}\right)^{5}-f_{0}^{5}\right] F_{0} \varphi_{\theta_{1}+2}\right| \\
& \lesssim\left|\int \partial_{x} q_{1}\left(F_{0} \varphi_{\theta_{1}+2}\right)_{x}\right|+\left|\int\left[\left(q_{1}+f_{0}\right)^{5}-f_{0}^{5}\right] F_{0} \varphi_{\theta_{1}+2}\right| \\
& \lesssim \frac{1}{100} \int\left(\partial_{x} q_{1}\right)^{2} \varphi_{\theta_{1}+2}^{\prime}+\frac{1}{100} \int q_{1}^{2} \varphi_{\theta_{1}}^{\prime} \\
& \quad+C \int\left(\left|\partial_{x} F_{0}\right|^{2} \frac{\varphi_{\theta_{1}+2}^{2}}{\varphi_{\theta_{1}+2}^{\prime}}+\left|F_{0}\right|^{2} \varphi_{\theta_{1}+2}^{\prime}+\left|F_{0}\right|^{2} \frac{f_{0}^{8} \varphi_{\theta_{1}+2}^{2}}{\varphi_{\theta_{1}}^{\prime}}\right) .
\end{aligned}
$$




$$
\begin{gathered}
2 \int \partial_{x}^{3} q_{1} \partial_{x} q_{1} \varphi_{\theta_{1}+2}^{\prime}=-2 \int\left(\partial_{x}^{2} q_{1}\right)^{2} \varphi_{\theta_{1}+2}^{\prime}+\int\left(\partial_{x} q_{1}\right)^{2} \varphi_{\theta_{1}+2}^{\prime \prime \prime} \\
\leq-2 \int\left(\partial_{x}^{2} q_{1}\right)^{2} \varphi_{\theta_{1}+2}^{\prime}+\frac{1}{4} \int\left(\partial_{x} q_{1}\right)^{2} \varphi_{\theta_{1}+2}^{\prime} . \\
\left|2 \int\left[\left(q_{1}+f_{0}\right)^{5}-f_{0}^{5}\right]_{x} \partial_{x} q_{1} \varphi_{\theta_{1}+2}^{\prime}\right| \\
=\left|10 \int\left(\partial_{x} q_{1}\right)^{2}\left(q_{1}+f_{0}\right)^{4} \varphi_{\theta_{1}+2}^{\prime}+10 \int\left(\left(q_{1}+f_{0}\right)^{4}-f_{0}^{4}\right) \partial_{x} f_{0} \partial_{x} q_{1} \varphi_{\theta_{1}+2}^{\prime}\right| \\
\leq \frac{1}{100} \int\left(\partial_{x} q_{1}\right)^{2} \varphi_{\theta_{1}+1}^{\prime}+C \int q_{1}^{2} \varphi_{\theta_{1}}^{\prime} \cdot \\
2\left|\int F_{0} \partial_{x} q_{1} \varphi_{\theta_{1}+2}^{\prime}\right| \leq \frac{1}{100} \int\left(\partial_{x} q_{1}\right)^{2} \varphi_{\theta_{1}+1}^{\prime}+C \int\left|F_{0}\right|^{2} \varphi_{\theta_{1}+2}^{\prime} .
\end{gathered}
$$

Combining these estimates, and using the expression of $F_{0}$ as in (A.6), we find

$$
E_{\theta_{1}}^{\prime}(t) \leq-\int\left(\partial_{x}^{2} q_{1}\right)^{2} \varphi_{\theta_{1}+2}^{\prime}-\frac{1}{10} \int\left(\partial_{x} q_{1}\right)^{2} \varphi_{\theta_{1}+2}^{\prime}+C \int q_{1}^{2} \varphi_{\theta_{1}}^{\prime}+C\left(t+x_{0}\right)^{\theta_{1}-2 \theta-4}
$$

By integration, and using (A.7),

$$
\begin{aligned}
& E_{\theta_{1}}(t)+\frac{1}{10} \int_{0}^{t} \int\left[\left(\partial_{x}^{2} q_{1}\right)^{2}+\left(\partial_{x} q_{1}\right)^{2}\right] \varphi_{\theta_{1}+2}^{\prime} \\
& \lesssim \begin{cases}\left(t+x_{0}\right)^{\theta_{1}-2 \theta-3} & \text { if } 0<\theta_{1}-2 \theta-3<1 \\
x_{0}^{\theta_{1}-2 \theta-3} & \text { if } \theta_{1}-2 \theta-3<0 .\end{cases}
\end{aligned}
$$

We look for an estimate on $\partial_{x} q_{1}(t)$ from the above estimate on $E_{\theta_{1}}(t)$. Note first that

$$
\begin{aligned}
\left\|q_{1}^{2} \sqrt{\varphi_{\theta_{1}+2}}\right\|_{L^{\infty}} & \lesssim \int_{x}^{+\infty}\left|q_{1}\right|\left|\partial_{x} q_{1}\right| \sqrt{\varphi_{\theta_{1}+2}}+\int_{x}^{+\infty}\left|q_{1}\right|^{2} \frac{\varphi_{\theta_{1}+2}^{\prime}}{\sqrt{\varphi_{\theta_{1}+2}}} \\
& \lesssim\left(\int q_{1}^{2}\right)^{\frac{1}{2}}\left(\int\left|\partial_{x} q_{1}\right|^{2} \varphi_{\theta_{1}+2}+\int\left|q_{1}\right|^{2} \varphi_{\theta_{1}}\right)^{\frac{1}{2}}
\end{aligned}
$$

so that

$$
\int q_{1}^{6} \varphi_{\theta_{1}+2} \leq\left\|q_{1}^{2} \sqrt{\varphi_{\theta_{1}+2}}\right\|_{L^{\infty}}^{2} \int q_{1}^{2} \lesssim\left(\int q_{1}^{2}\right)^{2}\left(\int\left|\partial_{x} q_{1}\right|^{2} \varphi_{\theta_{1}+2}+M_{\theta_{1}}(t)\right) .
$$

Also,

$$
\int q_{1}^{2} f_{0}^{4} \varphi_{\theta_{1}+2} \lesssim \int q_{1}^{2} \varphi_{\theta_{1}}=M_{\theta_{1}}(t)
$$


Thus,

$$
\begin{aligned}
E_{\theta_{1}}(t) & \geq \int\left(\partial_{x} q_{1}\right)^{2} \varphi_{\theta_{1}+2}-C \int\left(q_{1}^{6}+q_{1}^{2} f_{0}^{4}\right) \varphi_{\theta_{1}+2} \\
& \geq \frac{1}{2} \int\left(\partial_{x} q_{1}\right)^{2} \varphi_{\theta_{1}+2}-C M_{\theta_{1}}(t),
\end{aligned}
$$

and so

$$
\begin{aligned}
& \int\left(\partial_{x} q_{1}\right)^{2} \varphi_{\theta_{1}+2}+\int_{0}^{t} \int\left[\left(\partial_{x}^{2} q_{1}\right)^{2}+\left(\partial_{x} q_{1}\right)^{2}\right] \varphi_{\theta_{1}+2}^{\prime} \\
& \lesssim \begin{cases}\left(t+x_{0}\right)^{\theta_{1}-2 \theta-3} & \text { if } 0<\theta_{1}-2 \theta-3<1 \\
x_{0}^{\theta_{1}-2 \theta-3} & \text { if } \theta_{1}-2 \theta-3<0 .\end{cases}
\end{aligned}
$$

Note also that for any $x$,

$$
\begin{aligned}
q_{1}^{2}(t, x) \varphi_{\theta_{1}+1}\left(x-\frac{t}{4}-\frac{x_{0}}{4}\right) & \lesssim \int_{x}^{+\infty}\left|q_{1}\right|\left|\partial_{x} q_{1}\right| \varphi_{\theta_{1}+1}+\int_{x}^{+\infty}\left|q_{1}\right|^{2} \varphi_{\theta_{1}+1}^{\prime} \\
& \lesssim \int\left|\partial_{x} q_{1}\right|^{2} \varphi_{\theta_{1}+2}+\int\left|q_{1}\right|^{2} \varphi_{\theta_{1}}
\end{aligned}
$$

and, with $\theta_{1}=2 \theta+\frac{15}{4}$, using the properties of $\varphi_{\theta_{1}+1}$, for $x>\frac{1}{2}\left(t+x_{0}\right)$,

$$
\left|q_{1}(t, x)\right| \lesssim x^{-\left(\frac{\theta_{1}}{2}+\frac{1}{2}\right)} t^{\frac{3}{8}}=x^{-\left(\theta+\frac{19}{8}\right)} t^{\frac{3}{8}} \lesssim x^{-(\theta+2)}
$$

Finally, we briefly treat the case of higher order derivatives. We use an induction argument, assuming at the stage $k$ that for all $1 \leq k^{\prime}<k$, for all $x, t$

$$
\begin{aligned}
& \int_{0}^{t} \int\left(\partial_{x}^{k^{\prime}} q_{1}\right)^{2}(t) \varphi_{\theta_{1}+2 k^{\prime}}^{\prime}\left(x-\frac{t}{4}-\frac{x_{0}}{4}\right) d t \\
& +\int\left(\partial_{x}^{k^{\prime}} q_{1}(t)\right)^{2} \varphi_{\theta_{1}+2 k^{\prime}}\left(x-\frac{t}{4}-\frac{x_{0}}{4}\right) \\
& +\left|\partial_{x}^{k^{\prime}-1} q_{1}(t)\right|^{2} \varphi_{\theta_{1}+2 k^{\prime}-1}\left(x-\frac{t}{4}-\frac{x_{0}}{4}\right) \\
& \lesssim \begin{cases}\left(t+x_{0}\right)^{\theta_{1}-2 \theta-3} & \text { if } 0<\theta_{1}-2 \theta-3<1 \\
x_{0}^{\theta_{1}-2 \theta-3} & \text { if } \theta_{1}-2 \theta-3<0\end{cases}
\end{aligned}
$$

and we prove the same estimates for $k^{\prime}=k$ using $F_{\theta_{1}, k}$. 
Indeed, by simple computation:

$$
\begin{aligned}
F_{\theta_{1}, k}^{\prime}(t)= & 2 \int\left(\partial_{x}^{k} q_{1}\right)_{t}\left(\partial_{x}^{k} q_{1}\right) \varphi_{\theta_{1}+2 k}-\frac{1}{2} \int\left(\partial_{x}^{k} q_{1}\right)^{2} \varphi_{\theta_{1}+2 k}^{\prime} \\
= & -2 \int\left(\partial_{x}^{k+3} q_{1}\right)\left(\partial_{x}^{k} q_{1}\right) \varphi_{\theta_{1}+2 k}-2 \int \partial_{x}^{k+1}\left(\left(q_{1}+f_{0}\right)^{5}-f_{0}^{5}\right) \partial_{x}^{k} q_{1} \varphi_{\theta_{1}+2 k} \\
& -\frac{1}{2} \int\left(\partial_{x}^{k} q_{1}\right)^{2} \varphi_{\theta_{1}+2 k}^{\prime}+\int\left(\partial_{x}^{k} F_{0}\right)\left(\partial_{x}^{k} q_{1}\right) \varphi_{\theta_{1}+2 k} \\
\leq & -3 \int\left(\partial_{x}^{k+1} q_{1}\right)^{2} \varphi_{\theta_{1}+2 k}^{\prime}-\frac{1}{4} \int\left(\partial_{x}^{k} q_{1}\right)^{2} \varphi_{\theta_{1}+2 k}^{\prime} \\
& +2 \int \partial_{x}^{k}\left(\left(q_{1}+f_{0}\right)^{5}-f_{0}^{5}\right)\left(\left(\partial_{x}^{k+1} q_{1}\right) \varphi_{\theta_{1}+2 k}+\left(\partial_{x}^{k} q_{1}\right) \varphi_{\theta_{1}+2 k}^{\prime}\right) \\
& +\int\left(\partial_{x}^{k} F_{0}\right)\left(\partial_{x}^{k} q_{1}\right) \varphi_{\theta_{1}+2 k .}
\end{aligned}
$$

We claim, arguing as in (A.6), that

$$
\left|\int\left(\partial_{x}^{k} F_{0}\right)\left(\partial_{x}^{k} q_{1}\right) \varphi_{\theta_{1}+2 k}\right| \leq \frac{1}{100} \int\left(\partial_{x}^{k} q_{1}\right)^{2} \varphi_{\theta_{1}+2 k}^{\prime}+C t^{\theta_{1}-2 \theta-4} .
$$

Next, we claim that

$$
\begin{aligned}
& \left|\int \partial_{x}^{k}\left(\left(q_{1}+f_{0}\right)^{5}-f_{0}^{5}\right)\left(\left(\partial_{x}^{k+1} q_{1}\right) \varphi_{\theta_{1}+2 k}+\left(\partial_{x}^{k} q_{1}\right) \varphi_{\theta_{1}+2 k}^{\prime}\right)\right| \\
& \leq \frac{1}{100} \int\left(\partial_{x}^{k+1} q_{1}\right) \varphi_{\theta_{1}+2 k}^{\prime}+\frac{1}{100} \int\left(\partial_{x}^{k} q_{1}\right) \varphi_{\theta_{1}+2 k}^{\prime}+\sum_{k^{\prime}=0}^{k-1} \int\left(\partial_{x}^{k^{\prime}} q_{1}\right)^{2} \varphi_{\theta_{1}+2 k^{\prime}}^{\prime}
\end{aligned}
$$

Indeed, looking for example at the purely nonlinear term in $q_{1}$, we have

$$
\begin{aligned}
& \left|\int \partial_{x}^{k}\left(q_{1}^{5}\right)\left(\partial_{x}^{k+1} q_{1}\right) \varphi_{\theta_{1}+2 k}\right|+\left|\int \partial_{x}^{k}\left(q_{1}^{5}\right)\left(\partial_{x}^{k} q_{1}\right) \varphi_{\theta_{1}+2 k}^{\prime}\right| \\
& \leq C \int\left(\partial_{x}^{k}\left(q_{1}^{5}\right)\right)^{2} \frac{\varphi_{\theta_{1}+2 k}^{2}}{\varphi_{\theta_{1}+2 k}^{\prime}}+\frac{1}{200} \int\left(\partial_{x}^{k} q_{1}\right)^{2} \varphi_{\theta_{1}+2 k}^{\prime} \\
& \quad+\frac{1}{200} \int\left(\partial_{x}^{k+1} q_{1}\right)^{2} \varphi_{\theta_{1}+2 k}^{\prime},
\end{aligned}
$$


and

$$
\begin{aligned}
& \int\left(\partial_{x}^{k}\left(q_{1}^{5}\right)\right)^{2} \frac{\varphi_{\theta_{1}+2 k}^{2}}{\varphi_{\theta_{1}+2 k}^{\prime}} \\
& \lesssim \int\left(\partial_{x}^{k}\left(q_{1}^{5}\right)\right)^{2} \varphi_{\theta_{1}+2 k_{1}+2 k_{2}+2 k_{3}+2 k_{4}+2 k_{5}+1} \\
& \lesssim \sum_{\substack{k_{1}+k_{2}+k_{3}+k_{4}+k_{5}=k \\
k_{5} \geq k_{4} \geq k_{3} \geq k_{2} \geq k_{1}}}\left(\Pi_{l=1}^{3}\left\|\left(\partial_{x}^{k_{l}} q_{1}\right)^{2} \varphi_{2 k_{l}+2}\right\|_{L^{\infty}}\right)\left\|\left(\partial_{x}^{k_{4}} q_{1}\right)^{2} \varphi_{2 k_{4}-2}\right\|_{L^{\infty}} \int\left|\partial_{x}^{k_{5}} q_{1}\right|^{2} \varphi_{\theta_{1}+2 k_{5}-1}^{\prime} \\
& \lesssim \delta\left(x_{0}^{-1}\right) \sum_{k^{\prime}=0}^{k} \int\left(\partial_{x}^{k^{\prime}} q_{1}\right)^{2} \varphi_{\theta_{1}+2 k^{\prime}-1}^{\prime}
\end{aligned}
$$

where the $L^{\infty}$ norms above are estimated using (A.14). The other terms, all containing $f_{0}$, are similar and easier.

By integration of $F_{k}^{\prime}$ using (A.15) and (A.14), we obtain

$$
F_{\theta_{1}, k}(t)+\int_{0}^{t} \int\left(\partial_{x}^{k+1} q_{1}\right)^{2} \varphi_{\theta_{1}+2 k}^{\prime} \lesssim \begin{cases}\left(t+x_{0}\right)^{\theta_{1}-2 \theta-3} & \text { if } 0<\theta_{1}-2 \theta-3<1 \\ x_{0}^{\theta_{1}-2 \theta-3} & \text { if } \theta_{1}-2 \theta-3<0 .\end{cases}
$$

Arguing as in the proof of (A.12), we prove (A.14) for $k^{\prime}=k$. The induction argument being complete, we finish the proof as in (A.13).

\section{B. Proof of monotonicity results on $\varepsilon$}

\section{B.1. Proof of Lemma 3.3}

We compute from (2.39):

$$
\begin{gathered}
\frac{1}{2} \frac{d}{d s} \int \varphi_{10} \varepsilon^{2}=\int \varepsilon_{s} \varepsilon \varphi_{10} \\
=\int \varphi_{10} \varepsilon\left[\frac{\lambda_{s}}{\lambda} \Lambda \varepsilon+\left(-\varepsilon_{y y}+\varepsilon-\left(\varepsilon+p Y_{0}+Q_{b}\right)^{5}+Q_{b}^{5}+p 5 Q^{4} Y_{0}\right)_{y}\right. \\
\quad-p_{s} Y_{0}+\left(5 Q^{4}(p-q)\right)_{y}+\frac{\lambda_{s}}{\lambda} p \Lambda Y_{0}+\left(\frac{\lambda_{s}}{\lambda}+b\right) \Lambda Q_{b} \\
\left.+\left(\frac{x_{s}}{\lambda}-1\right)\left(Q_{b}+\varepsilon+p Y_{0}\right)_{y}+\Phi_{b}+\Psi_{b}-W_{y}\right] .
\end{gathered}
$$


We integrate by parts the linear term and use $y \varphi_{10}^{\prime}=10 \varphi_{10}$ for $y \geq 1$ and $\varphi_{10}^{\prime \prime \prime} \ll$ $\varphi_{10}^{\prime}$ for $y$ large enough to derive the bound

$$
\begin{aligned}
& \int \varphi_{10} \varepsilon\left[\frac{\lambda_{s}}{\lambda} \Lambda \varepsilon+\left(-\varepsilon_{y y}+\varepsilon\right)_{y}\right] \\
= & -\frac{1}{2} \frac{\lambda_{s}}{\lambda} \int y \varphi_{10}^{\prime} \varepsilon^{2}-\frac{3}{2} \int \varphi_{10}^{\prime} \varepsilon_{y}^{2}-\frac{1}{2} \int \varphi_{10}^{\prime} \varepsilon^{2}+\frac{1}{2} \int \varphi_{10}^{\prime \prime \prime} \varepsilon^{2} \\
\leq & -\frac{10}{2} \frac{\lambda_{s}}{\lambda} \int \varphi_{10} \varepsilon^{2}-\frac{1}{4} \int \varphi_{10}^{\prime}\left(\varepsilon_{y}^{2}+\varepsilon^{2}\right)+C \mathcal{N}_{1, \mathrm{loc} .}
\end{aligned}
$$

Integrating by parts in the nonlinear term, we can remove all derivatives on $\varepsilon$ to obtain (using $\left|Q_{b}\right|+\left|\left(Q_{b}\right)_{y}\right| \leq C e^{-\frac{1}{2} y}$ for $y>0$ )

$$
\begin{aligned}
\left|\int \varphi_{10} \varepsilon\left[\left(\varepsilon+Q_{b}\right)^{5}-Q_{b}^{5}\right]_{y}\right| & \lesssim \int_{y>0} \varphi_{10} e^{-\frac{1}{2} y} \varepsilon^{2}\left(|\varepsilon|^{3}+1\right)+\int \varphi_{10}^{\prime} \varepsilon^{6} \\
& \lesssim \int_{y>0} e^{-\frac{1}{4} y} \varepsilon^{2}\left(|\varepsilon|^{3}+1\right)+\int \varphi_{10}^{\prime} \varepsilon^{6} .
\end{aligned}
$$

Thus, by standard Sobolev estimates,

$$
\left|\int \varphi_{10} \varepsilon\left[\left(\varepsilon+Q_{b}\right)^{5}-Q_{b}^{5}\right]_{y}\right| \lesssim \mathcal{N}_{1, \text { loc }}+\delta\left(\alpha^{*}\right) \int \varphi_{10}^{\prime}\left(\varepsilon_{y}^{2}+\varepsilon^{2}\right) .
$$

Next, by the bootstrap estimates,

$$
\begin{aligned}
& \left|\int \varphi_{10} \varepsilon\left[\left(\varepsilon+Q_{b}+p Y_{0}\right)^{5}-5 p Q^{4} Y_{0}-\left(\varepsilon+Q_{b}\right)^{5}\right]_{y}\right| \\
& \lesssim \int_{y>10} \varepsilon e^{-\frac{1}{2} y}\left(p^{2}+|b||p|+|p|\left(|\varepsilon|+|\varepsilon|^{5}\right) \lesssim \mathcal{N}_{1, \text { loc }}+\frac{1}{s^{2}} .\right.
\end{aligned}
$$

By (3.15) and $Y_{0} \in \mathcal{Y}$,

$$
\left|p_{s} \int Y_{0} \varphi_{10} \varepsilon\right| \lesssim \mathcal{N}_{1, \text { loc }}+\frac{1}{s^{2}}
$$

By (2.42) and (3.12),

$$
\begin{aligned}
\left|\int 5\left(Q^{4}(p-q)\right)_{y} \varepsilon \varphi_{10}\right| & =\left|\int 5(p-q) e^{-4|y|}\left(\varepsilon \varphi_{10}\right)_{y}\right| \\
& \leq C \frac{1}{s^{2}} \int\left(\left|\varepsilon_{y}\right|+|\varepsilon|\right) e^{-|y|} \varphi_{10} \\
& \leq \frac{1}{100} \int \varepsilon_{y}^{2} \varphi_{10}^{\prime}+C \mathcal{N}_{1, \text { loc }}+\frac{C}{s^{2}} .
\end{aligned}
$$


By (3.14),

$$
\left|\frac{\lambda_{s}}{\lambda} p \int \Lambda Y_{0} \varphi_{10} \varepsilon\right| \lesssim \mathcal{N}_{1, \text { loc }}+\frac{1}{s^{2}} .
$$

The terms involving the geometric parameters are controlled from the exponential localization of $Q_{b}$ on the right and (3.13)-(3.14):

$$
\begin{aligned}
\left|\frac{\lambda_{s}}{\lambda}+b\right|\left|\int \varphi_{10} \varepsilon\left(\Lambda Q_{b}\right)\right| & \lesssim\left(\frac{1}{s^{2}}+\mathcal{N}_{1, \mathrm{loc}}^{\frac{1}{2}}\right) \mathcal{N}_{i, \mathrm{loc}}^{\frac{1}{2}} \lesssim \mathcal{N}_{1, \mathrm{loc}}+\frac{1}{s^{2}}, \\
\left|\frac{x_{s}}{\lambda}-1\right|\left|\int \varphi_{10} \varepsilon\left(Q_{b}+\varepsilon+p Y_{0}\right)_{y}\right| & \lesssim\left(\frac{1}{s^{2}}+\mathcal{N}_{1, \mathrm{loc}}^{\frac{1}{2}}\right)\left[\mathcal{N}_{1, \mathrm{loc}}^{\frac{1}{2}}+\int \varphi_{10}^{\prime} \varepsilon^{2}\right] \\
& \lesssim \mathcal{N}_{1, \mathrm{loc}}+\frac{1}{s^{2}}+\delta\left(\alpha^{*}\right) \int \varphi_{10}^{\prime} \varepsilon^{2}, \\
\int\left|\varphi_{10} \varepsilon \Phi_{b}\right| & \lesssim\left|b_{s}\right| \mathcal{N}_{1, \mathrm{loc}}^{\frac{1}{2}} \lesssim \frac{1}{s^{2}}+\mathcal{N}_{1, \text { loc }} .
\end{aligned}
$$

We control similarily the interaction with the error from (2.10):

$$
\int\left|\varphi_{10} \varepsilon \Psi_{b}\right| \lesssim \frac{1}{s^{2}} \mathcal{N}_{1, \text { loc }}^{\frac{1}{2}} \lesssim \frac{1}{s^{2}}+\mathcal{N}_{1, \text { loc }}
$$

Finally, we claim that

$$
\left|\int \varphi_{10} \varepsilon W_{y}\right| \leq C \mathcal{N}_{1, \mathrm{loc}}+\frac{C}{s^{2}}+\frac{1}{50} \int \varepsilon^{2} \varphi_{10}^{\prime} .
$$

We only treat the first term in $W$, the other terms are similar and easier. First, integrating by parts, we remove the derivative from $\varepsilon$ to obtain derivative on $q, \varphi_{10}$. Indeed,

$$
\begin{aligned}
- & \int\left[5\left(Q_{b}+\varepsilon+p Y_{0}\right)^{4} q-5 Q^{4} q\right]_{y} \varepsilon \varphi_{10}=\int\left[5\left(Q_{b}+\varepsilon+p Y_{0}\right)^{4} q-5 Q^{4} q\right]\left(\varepsilon \varphi_{10}\right)_{y} \\
= & \int\left\{\left[\left(Q_{b}+\varepsilon+p Y_{0}\right)^{5}-\left(Q_{b}+p Y_{0}\right)^{5}-5 Q^{4} \varepsilon\right]_{y}\right. \\
& \left.-5\left(Q_{b}+p Y_{0}\right)_{y}\left[\left(Q_{b}+\varepsilon+p Y_{0}\right)^{4}-\left(Q_{b}+p Y_{0}\right)^{4}\right]+20 Q^{3} Q_{y} \varepsilon\right\} q \varphi_{10} \\
& +5 \int\left[\left(Q_{b}+\varepsilon+p Y_{0}\right)^{4}-Q^{4}\right] \varepsilon q \varphi_{10}^{\prime} \\
= & -\int\left[\left(Q_{b}+\varepsilon+p Y_{0}\right)^{5}-\left(Q_{b}+p Y_{0}\right)^{5}-5 Q^{4} \varepsilon\right]\left(q_{y} \varphi_{10}+q \varphi_{10}^{\prime}\right) \\
& -5 \int\left\{\left(Q_{b}+p Y_{0}\right)_{y}\left[\left(Q_{b}+\varepsilon+p Y_{0}\right)^{4}-\left(Q_{b}+p Y_{0}\right)^{4}\right]-4 Q^{3} Q_{y} \varepsilon\right\} q \varphi_{10} \\
& +5 \int\left[\left(Q_{b}+\varepsilon+p Y_{0}\right)^{4}-Q^{4}\right] \varepsilon q \varphi_{10}^{\prime} .
\end{aligned}
$$


From the above expression, we obtain for $s$ large enough (using $\|\varepsilon\|_{L^{\infty}} \lesssim\|\varepsilon\|_{H^{1}} \lesssim$ $\delta\left(\alpha^{*}\right)$ and $\left.\|q\|_{L^{\infty}(y>0)} \lesssim \frac{1}{s}\right)$

$$
\begin{aligned}
& \left|\int\left[5\left(Q_{b}+\varepsilon+p Y_{0}\right)^{4} q-5 Q^{4} q\right]_{y} \varepsilon \varphi_{10}\right| \\
& \lesssim \int\left(|q|+\left|q_{y}\right|\right) e^{-\frac{|y|}{2}}(|\varepsilon|+|p|+|b|)|\varepsilon|+\int\left(\left|q_{y}\right| \varphi_{10}+|q| \varphi_{10}^{\prime}\right)|\varepsilon|^{5} \\
& \leq C \mathcal{N}_{1, \text { loc }}+\frac{C}{s^{2}}+\frac{1}{100} \int \varepsilon^{2} \varphi_{10}^{\prime}+\delta\left(\alpha^{*}\right) \int|\varepsilon|^{2}\left|q_{y}\right| \varphi_{10} .
\end{aligned}
$$

To control the last term above, we use $q_{y}(t, y)=\lambda^{\frac{3}{2}} \partial_{x} q_{0}(t, \lambda y+x(t))$ so that $\left|q_{y}(t, y)\right| \lesssim \lambda^{\frac{3}{2}}(\lambda y+x(t))^{-\theta-1} \lesssim \lambda^{\frac{1}{2}} x^{-\theta}(t)(y+1)^{-1}$, for $y>10$, and

$$
\int|\varepsilon|^{2}\left|q_{y}\right| \varphi_{10} \lesssim \frac{\lambda^{\frac{1}{2}}}{x^{\theta}} \int_{y>0} \varepsilon^{2}(y+1)^{-1} \varphi_{10} \leq \frac{1}{100} \int \varepsilon^{2} \varphi_{10}^{\prime} .
$$

The collection of above estimates yields the bound:

$$
\frac{d}{d s} \int \varphi_{10} \varepsilon^{2}+10 \frac{\lambda_{s}}{\lambda} \int \varphi_{10} \varepsilon^{2} \lesssim \mathcal{N}_{1, \text { loc }}+\frac{1}{s^{2}},
$$

and (3.19) is proved.

\section{B.2. Proof of Lemma 3.4}

Step 1 . Weighted $L^{2}$ controls at the right.

We first recall from [38, proof of Proposition 3.1], the following controls for all $s \in\left[0, s_{0}\right]$,

$$
\begin{gathered}
\int_{y>0} y \varepsilon^{2}(s) \lesssim\left(1+\frac{1}{\lambda^{\frac{10}{9}}(s)}\right) \mathcal{N}_{1, \mathrm{loc}}^{\frac{8}{9}}(s), \\
\int_{y>0} y^{2} \varepsilon^{2}(s) \lesssim\left(1+\frac{1}{\lambda^{\frac{10}{9}}(s)}\right) \mathcal{N}_{2, \mathrm{loc}}^{\frac{8}{9}}(s), \\
\int_{y>0}|\varepsilon(s)| \lesssim \mathcal{N}_{2}^{\frac{1}{2}}(s) .
\end{gathered}
$$

Step 2. Algebraic computations on $\mathcal{F}_{i}$.

First, note that the equation of $\varepsilon$ (2.39) can be rewritten as

$$
\begin{aligned}
\partial_{s} \varepsilon-\frac{\lambda_{s}}{\lambda} \Lambda \varepsilon= & \left(-\partial_{y}^{2} \varepsilon+\varepsilon-Z\right)_{y} \\
& -p_{s} Y_{0}+\left(5 Q^{4}(p-q)\right)_{y}+\frac{\lambda_{s}}{\lambda} p \Lambda Y_{0}+\left(\frac{\lambda_{s}}{\lambda}+b\right) \Lambda Q_{b} \\
& +\left(\frac{x_{s}}{\lambda}-1\right)\left(Q_{b}+\varepsilon+p Y_{0}\right)_{y}+\Phi_{b}+\Psi_{b},
\end{aligned}
$$


where

$$
\begin{aligned}
Z & =\left(Q_{b}+p Y_{0}+q+\varepsilon\right)^{5}-Q_{b}^{5}-5 Q^{4}\left(p Y_{0}+q\right)-q^{5}, \\
\Phi_{b} & =-b_{s}\left(\chi_{b}+\gamma y\left(\chi_{b}\right)_{y}\right) P, \quad-\Psi_{b}=\left(Q_{b}^{\prime \prime}-Q_{b}+Q_{b}^{5}\right)^{\prime}+b \Lambda Q_{b} .
\end{aligned}
$$

We compute

$$
\begin{aligned}
& s^{-j} \frac{d}{d s}\left[s^{j} \mathcal{F}_{\rangle}\right]=2 \int \psi_{B}\left(\varepsilon_{y}\right)_{s} \varepsilon_{y}+2 \int \varepsilon_{s}\left[\varepsilon \varphi_{i, B}-\psi_{B} Z\right] \\
& -2 \int \psi_{B}\left(Q_{b}\right)_{s}\left[\left(\varepsilon+Q_{b}+p Y_{0}+q\right)^{5}-\left(Q_{b}+p Y_{0}+q\right)^{5}-5 \varepsilon Q_{b}^{4}\right] \\
& -2 \int \psi_{B}\left(p_{s} Y_{0}+q_{s}\right)\left[\left(\varepsilon+Q_{b}+p Y_{0}+q\right)^{5}-\left(Q_{b}+p Y_{0}+q\right)^{5}-5 \varepsilon Q^{4}\right] \\
& +10 \int \psi_{B} q_{s} q^{4} \varepsilon+\frac{j}{s} \mathcal{F}_{i}
\end{aligned}
$$

which we rewrite as

$$
s^{-j} \frac{d}{d s}\left[s^{j} \mathcal{F}_{\rangle}\right]=f_{1}^{(i)}+f_{2}^{(i)}+f_{3}^{(i)},
$$

where

$$
\begin{aligned}
f_{1}^{(i)}= & 2 \int\left(\varepsilon_{s}-\frac{\lambda_{s}}{\lambda} \Lambda \varepsilon\right)\left(-\left(\psi_{B} \varepsilon_{y}\right)_{y}+\varepsilon \varphi_{i, B}-\psi_{B} Z\right) \\
f_{2}^{(i, j)}= & 2 \frac{\lambda_{s}}{\lambda} \int \Lambda \varepsilon\left(-\left(\psi_{B} \varepsilon_{y}\right)_{y}+\varepsilon \varphi_{i, B}-\psi_{B} Z\right)+\frac{j}{s} \mathcal{F}_{i} \\
f_{3}^{(i)}= & -2 \int \psi_{B}\left(Q_{b}\right)_{s}\left[\left(\varepsilon+Q_{b}+p Y_{0}+q\right)^{5}-\left(Q_{b}+p Y_{0}+q\right)^{5}-5 \varepsilon Q_{b}^{4}\right] \\
& -2 \int \psi_{B}\left(p_{s} Y_{0}+q_{s}\right)\left[\left(\varepsilon+Q_{b}+p Y_{0}+q\right)^{5}-\left(Q_{b}+p Y_{0}+q\right)^{5}-5 \varepsilon Q^{4}\right] \\
& +10 \int \psi_{B} q_{s} q^{4} \varepsilon .
\end{aligned}
$$

We claim the following estimates on the above terms: for some $\mu_{0}>0$,

$$
\begin{aligned}
\frac{d}{d s} f_{1}^{(i)} & \leq-\mu_{0} \int\left(\varepsilon_{y}^{2}+\varepsilon^{2}\right) \varphi_{i, B}^{\prime}+C s^{-4} \\
\left|\frac{d}{d s} f_{k}^{(i)}\right| & \leq \frac{\mu_{0}}{10} \int\left(\varepsilon_{y}^{2}+\varepsilon^{2}\right) \varphi_{i, B}^{\prime}+C s^{-4}+C s^{10 \beta-9}, \quad \text { for } k=2,3 .
\end{aligned}
$$

Inserting (B.7) and (B.8) into (B.6) yields (3.21) for all $j$. In Steps 3-5, we prove (B.7) and (B.8). 
Observe that the definitions of $\varphi_{i}$ and $\psi$ imply the following estimates:

$\forall y \in \mathbb{R},\left|\varphi_{i}^{\prime \prime \prime}(y)\right|+\left|\varphi_{i}^{\prime \prime}(y)\right|+\left|\psi^{\prime \prime \prime}(y)\right|+\left|y \psi^{\prime}(y)\right|+|\psi(y)| \lesssim \varphi_{i}^{\prime}(y) \lesssim \varphi_{i}(y)$,

$\forall y \in(-\infty, 2], \quad e^{|y|} \psi(y)+e^{|y|} \psi^{\prime}(y)+\varphi_{i}(y) \lesssim \varphi_{i}^{\prime}(y)$,

$\forall y \in \mathbb{R}, \quad \varphi_{2}^{\prime}(y) \lesssim \varphi_{1}(y) \lesssim \varphi_{2}^{\prime}(y)$.

In particular,

$\mathcal{N}_{1, \mathrm{loc}}(s) \lesssim \mathcal{N}_{2, \mathrm{loc}}(s) \lesssim \mathcal{N}_{1}(s) \lesssim \mathcal{N}_{2}(s), \quad \int \varepsilon^{2}(s, y) \varphi_{1, B}(y) d y \lesssim \mathcal{N}_{2, \text { loc }}(s)$

Step 3. Control of $f_{1}^{(i)}$. Proof of (B.7). We compute $f_{1}^{(i)}$ using (B.5)

$$
\begin{aligned}
\mathrm{f}_{1}^{(i)}= & 2 \int\left(-\varepsilon_{y y}+\varepsilon-Z\right)_{y}\left(-\left(\psi_{B} \varepsilon_{y}\right)_{y}+\varepsilon \varphi_{i, B}-\psi_{B} Z\right) \\
& +2\left(\frac{\lambda_{s}}{\lambda}+b\right) \int \Lambda Q_{b}\left(-\left(\psi_{B} \varepsilon_{y}\right)_{y}+\varepsilon \varphi_{i, B}-\psi_{B} Z\right) \\
& +2\left(\frac{x_{s}}{\lambda}-1\right) \int\left(Q_{b}+\varepsilon+p Y_{0}\right)_{y}\left(-\left(\psi_{B} \varepsilon_{y}\right)_{y}+\varepsilon \varphi_{i, B}-\psi_{B} Z\right) \\
& +2 \int \Phi_{b}\left(-\left(\psi_{B} \varepsilon_{y}\right)_{y}+\varepsilon \varphi_{i, B}-\psi_{B} Z\right) \\
& +2 \int \Psi_{b}\left(-\left(\psi_{B} \varepsilon_{y}\right)_{y}+\varepsilon \varphi_{i, B}-\psi_{B} Z\right) \\
& +\int\left(-p_{s} Y_{0}+\left(5 Q^{4}(p-q)\right)_{y}+\frac{\lambda_{s}}{\lambda} p \Lambda Y_{0}\right)\left(-\left(\psi_{B} \varepsilon_{y}\right)_{y}+\varepsilon \varphi_{i, B}-\psi_{B} Z\right) \\
= & \mathrm{f}_{1,1}^{(i)}+\mathrm{f}_{1,2}^{(i)}+\mathrm{f}_{1,3}^{(i)}+\mathrm{f}_{1,4}^{(i)}+\mathrm{f}_{1,5}^{(i)}+\mathrm{f}_{1,6}^{(i)} .
\end{aligned}
$$

$\underline{\operatorname{Term} f_{1,1}^{(i)}}$ : We first integrate by parts

$$
\begin{aligned}
f_{1,1}^{(i)}= & 2 \int\left[-\varepsilon_{y y}+\varepsilon-Z\right]_{y}\left[-\varepsilon_{y y}+\varepsilon-Z\right] \psi_{B} \\
& +2 \int\left[-\varepsilon_{y y}+\varepsilon-Z\right]_{y}\left(-\psi_{B}^{\prime} \varepsilon_{y}+\varepsilon\left(\varphi_{i, B}-\psi_{B}\right)\right) .
\end{aligned}
$$

We compute the various terms

$$
\begin{aligned}
2 \int & {\left[-\varepsilon_{y y}+\varepsilon-Z\right]_{y}\left[-\varepsilon_{y y}+\varepsilon-Z\right] \psi_{B}=-\int \psi_{B}^{\prime}\left[-\varepsilon_{y y}+\varepsilon-Z\right]^{2} } \\
= & -\int \psi_{B}^{\prime}\left[-\varepsilon_{y y}+\varepsilon\right]^{2}-\int \psi_{B}^{\prime}\left\{\left[-\varepsilon_{y y}+\varepsilon-Z\right]^{2}-\left[-\varepsilon_{y y}+\varepsilon\right]^{2}\right\} \\
= & -\left[\int \psi_{B}^{\prime}\left(\varepsilon_{y y}^{2}+2 \varepsilon_{y}^{2}\right)+\int \varepsilon^{2}\left(\psi_{B}^{\prime}-\psi_{B}^{\prime \prime \prime}\right)\right] \\
& -\int \psi_{B}^{\prime}\left\{\left[-\varepsilon_{y y}+\varepsilon-Z\right]^{2}-\left[-\varepsilon_{y y}+\varepsilon\right]^{2}\right\} .
\end{aligned}
$$


Next after integration by parts:

$$
\begin{gathered}
2 \int\left[-\varepsilon_{y y}+\varepsilon\right]_{y}\left[-\psi_{B}^{\prime} \varepsilon_{y}+\varepsilon\left(\varphi_{i, B}-\psi_{B}\right)\right] \\
=-2\left\{\int \psi_{B}^{\prime} \varepsilon_{y y}^{2}+\int \varepsilon_{y}^{2}\left(\frac{3}{2} \varphi_{i, B}^{\prime}-\frac{1}{2} \psi_{B}^{\prime}-\frac{1}{2} \psi_{B}^{\prime \prime \prime}\right)\right. \\
\left.\quad+\int \varepsilon^{2}\left(\frac{1}{2}\left(\varphi_{i, B}-\psi_{B}\right)^{\prime}-\frac{1}{2}\left(\varphi_{i, B}-\psi_{B}\right)^{\prime \prime \prime}\right)\right\} \\
-2 \int Z_{y}\left(\varphi_{i, B}-\psi_{B}\right) \varepsilon=2 \int Z\left(\varphi_{i, B}^{\prime}-\psi_{B}^{\prime}\right) \varepsilon+2 \int Z\left(\varphi_{i, B}-\psi_{B}\right) \varepsilon_{y} \\
=2 \int Z\left(\varphi_{i, B}^{\prime}-\psi_{B}^{\prime}\right) \varepsilon-\frac{1}{3} \int\left(\varphi_{i, B}-\psi_{B}\right)^{\prime} \\
\quad\left\{\left[\left(Q_{b}+\varepsilon+p Y_{0}+q\right)^{6}-\left(Q_{b}+p Y_{0}+q\right)^{6}-6 Q_{b}^{5} \varepsilon-6 q^{5} \varepsilon-30 Q^{4}\left(p Y_{0}+q\right) \varepsilon\right\}\right. \\
\quad-2 \int\left(\varphi_{i, B}-\psi_{B}\right)\left(Q_{b}\right)_{y}\left[\left(Q_{b}+\varepsilon+p Y_{0}+q\right)^{5}-\left(Q_{b}+p Y_{0}+q\right)^{5}-5 Q_{b}^{4} \varepsilon\right] \\
+40 \int\left(\varphi_{i, B}-\psi_{B}\right) Q^{\prime} Q^{3}\left(p Y_{0}+q\right) \varepsilon \\
\quad-2 \int\left(\varphi_{i, B}-\psi_{B}\right)\left(p Y_{0}^{\prime}+q_{y}\right)\left[\left(Q_{b}+\varepsilon+p Y_{0}+q\right)^{5}-\left(Q_{b}+p Y_{0}+q\right)^{5}-5 Q^{4} \varepsilon\right] .
\end{gathered}
$$

We collect the above computations and obtain the following

$$
\begin{aligned}
& f_{1,1}^{(i)}=-\int\left[3 \psi_{B}^{\prime} \varepsilon_{y y}^{2}+\left(3 \varphi_{i, B}^{\prime}+\psi_{B}^{\prime}-\psi_{B}^{\prime \prime \prime}\right) \varepsilon_{y}^{2}+\left(\varphi_{i, B}^{\prime}-\varphi_{i, B}^{\prime \prime \prime}\right) \varepsilon^{2}\right] \\
& -\frac{1}{3} \int\left[\left(\varepsilon+Q_{b}+p Y_{0}+q\right)^{6}-\left(Q_{b}+p Y_{0}+q\right)^{6}-6 Q_{b}^{5} \varepsilon-6 q^{5} \varepsilon\right. \\
& \left.\quad-30 Q^{4}\left(p Y_{0}+q\right) \varepsilon-6 Z \varepsilon\right]\left(\varphi_{i, B}^{\prime}-\psi_{B}^{\prime}\right) \\
& -2 \int\left(\varphi_{i, B}-\psi_{B}\right)\left(Q_{b}\right)_{y}\left[\left(Q_{b}+\varepsilon+p Y_{0}+q\right)^{5}-\left(Q_{b}+p Y_{0}+q\right)^{5}-5 Q_{b}^{4} \varepsilon\right] \\
& +40 \int\left(\varphi_{i, B}-\psi_{B}\right) Q^{\prime} Q^{3}\left(p Y_{0}+q\right) \varepsilon \\
& -2 \int\left(\varphi_{i, B}-\psi_{B}\right)\left(p Y_{0}^{\prime}+q_{y}\right)\left[\left(Q_{b}+\varepsilon+p Y_{0}+q\right)^{5}-\left(Q_{b}+p Y_{0}+q\right)^{5}-5 Q^{4} \varepsilon\right] \\
& +2 \int Z_{y} \varepsilon_{y} \psi_{B}^{\prime}-\int \psi_{B}^{\prime}\left\{\left[-\varepsilon_{y y}+\varepsilon-Z\right]^{2}-\left[-\varepsilon_{y y}+\varepsilon\right]^{2}\right\} \\
& =\left(f_{1,1}^{(i)}\right)^{<}+\left(f_{1,1}^{(i)}\right)^{\sim}+\left(f_{1,1}^{(i)}\right)^{>}
\end{aligned}
$$

where $\left(f_{1,1}^{(i)}\right)^{<, \sim,>}$ respectively corresponds to integration on $y<-\frac{B}{2},|y| \leq \frac{B}{2}$, $y>\frac{B}{2}$. We recall

$$
\|\varepsilon\|_{L^{\infty}} \lesssim\|\varepsilon\|_{H^{1}} \lesssim \delta\left(\alpha^{*}\right)
$$


- For the region $y<-B / 2$, we rely on monotonicity type arguments and estimate using (B.9):

$$
\begin{aligned}
& \int_{y<-B / 2} \varepsilon^{2}\left|\varphi_{i, B}^{\prime \prime \prime}\right| \lesssim \frac{1}{B^{2}} \int_{y<-B / 2} \varepsilon^{2} \varphi_{i, B}^{\prime} \leq \frac{1}{100} \int_{y<-B / 2} \varepsilon^{2} \varphi_{i, B}^{\prime}, \\
& \int_{y<-B / 2} \varepsilon_{y}^{2}\left|\psi_{B}^{\prime \prime \prime}\right| \lesssim \frac{1}{B^{2}} \int_{y<-B / 2} \varepsilon_{y}^{2} \varphi_{i, B}^{\prime} \leq \frac{1}{100} \int_{y<-B / 2} \varepsilon_{y}^{2} \varphi_{i, B}^{\prime},
\end{aligned}
$$

by choosing $B$ large enough. By (B.13) (for $B$ large and $\alpha^{*}$ small)

$$
\begin{aligned}
& \mid \int_{y<-B / 2}\left[\left(\varepsilon+Q_{b}+p Y_{0}+q\right)^{6}-\left(Q_{b}+p Y_{0}+q\right)^{6}-6 Q_{b}^{5} \varepsilon-6 q^{5} \varepsilon\right. \\
& \left.-30 Q^{4}\left(p Y_{0}+q\right) \varepsilon-6 Z \varepsilon\right]\left(\varphi_{i, B}^{\prime}-\psi_{B}^{\prime}\right) \\
& \lesssim \int_{y<-B / 2}\left(\delta\left(\alpha^{*}\right)+\left(\left|Q_{b}\right|^{4}+|p|^{4}+|q|^{4}\right) \varepsilon^{2}+(|b|+|p|+|q|)^{2}|\varepsilon|\right) \varphi_{i, B}^{\prime} \\
& \lesssim\left(\delta\left(\alpha^{*}\right)+\delta\left(s_{0}^{-1}\right)+e^{-\frac{B}{10}}\right) \int_{y<-B / 2} \varphi_{i, B}^{\prime} \varepsilon^{2}+\frac{1}{s^{2}}\left(\int_{y<-B / 2} \varepsilon^{2} \varphi_{i, B}^{\prime}\right)^{\frac{1}{2}} \\
& \leq \frac{1}{100} \int_{y<-B / 2} \varepsilon^{2} \varphi_{i, B}^{\prime}+\frac{1}{s^{4}}
\end{aligned}
$$

where we have used from the definition of $q$ and (3.12) the relation

$$
\left(\int q^{4} \varphi_{i, B}^{\prime}\right)^{\frac{1}{2}} \lesssim \frac{1}{s^{2}} .
$$

Similarily for small $\alpha^{*}$ depending on $B$,

$$
\begin{aligned}
& \mid \int_{y<-\frac{B}{2}}\left(\varphi_{i, B}-\psi_{B}\right)\left(Q_{b}\right)_{y}\left[\left(Q_{b}+\varepsilon+p Y_{0}+q\right)^{5}-\left(Q_{b}+p Y_{0}+q\right)^{5}-5 Q_{b}^{4} \varepsilon\right] \\
& -20 \int\left(\varphi_{i, B}-\psi_{B}\right) Q^{\prime} Q^{3}\left(p Y_{0}+q\right) \varepsilon \mid \\
& \lesssim B \int_{y<-\frac{B}{2}}\left(|\varepsilon|^{5}+\varepsilon^{2}\left(\left|Q_{b}\right|^{3}+|p|^{3}+|q|^{3}\right)\right)\left(\left|Q_{y}\right|+|b|\left|\left(P \chi_{b}\right)^{\prime}\right|\right) \varphi_{i, B}^{\prime} \\
& +B \int|\varepsilon|\left(|p|^{2}+|q|^{2}\right)\left(\left|Q_{y}\right|+|b|\left|\left(P \chi_{b}\right)^{\prime}\right|\right) \varphi_{i, B}^{\prime} \\
& +B \int_{|\varepsilon|(|p|+|q|)\left|\left(Q_{b}\right)_{y} Q_{b}^{3}-Q^{\prime} Q^{3}\right| \varphi_{i, B}^{\prime}} \\
& \leq \frac{1}{100} \int_{y<-B / 2}\left(\varepsilon_{y}^{2}+\varepsilon^{2}\right) \varphi_{i, B}^{\prime}+\frac{1}{s^{4}} .
\end{aligned}
$$


The next term in $\left(f_{1,1}^{(i)}\right)^{<}$is

$-2 \int\left(\varphi_{i, B}-\psi_{B}\right)\left(p Y_{0}^{\prime}+q_{y}\right)\left[\left(Q_{b}+\varepsilon+p Y_{0}+q\right)^{5}-\left(Q_{b}+p Y_{0}+q\right)^{5}-5 Q^{4} \varepsilon\right]$.

To estimate it, we note the following:

$$
\begin{aligned}
& \left|\left(Q_{b}+\varepsilon+p Y_{0}+q\right)^{5}-\left(Q_{b}+p Y_{0}+q\right)^{5}-5 Q^{4} \varepsilon\right| \\
& \lesssim|\varepsilon|^{5}+|\varepsilon|^{2}+|\varepsilon|(|b|+|p|+|q|) .
\end{aligned}
$$

Now, using

$$
\int|p| \varphi_{i, B} d y \lesssim \frac{1}{s}, \quad \int\left|q_{y}\right| \varphi_{i, B} d y \lesssim \frac{1}{s^{2}},
$$

we obtain proceeding as before

$$
\begin{aligned}
& \left|\int\left(\varphi_{i, B}-\psi_{B}\right)\left(p Y_{0}^{\prime}+q_{y}\right)\left[\left(Q_{b}+\varepsilon+p Y_{0}+q\right)^{5}-\left(Q_{b}+p Y_{0}+q\right)^{5}-5 Q^{4} \varepsilon\right]\right| \\
& \lesssim \frac{1}{100} \int_{y<-B / 2}\left(\varepsilon_{y}^{2}+\varepsilon^{2}\right) \varphi_{i, B}^{\prime}+\frac{1}{s^{4}} .
\end{aligned}
$$

We further estimate using (B.13) and $\left(\varphi_{i}^{\prime}\right)^{2} \lesssim \psi^{\prime} \lesssim\left(\varphi_{i}^{\prime}\right)^{2}$ for $y<-\frac{1}{2}$ :

$$
\begin{aligned}
& \left|\int Z_{y} \varepsilon_{y} \psi_{b}^{\prime}\right| \\
& \lesssim\left|\int_{y<-\frac{B}{2}} \psi_{B}^{\prime} \varepsilon_{y}\left\{\left(Q_{b}\right)_{y}\left[\left(Q_{b}+\varepsilon+p Y_{0}+q\right)^{4}-Q_{b}^{4}\right]-Q^{\prime} Q^{3}\left(p Y_{0}+q\right)\right\}\right| \\
& +\left|\int_{y<-\frac{B}{2}} \psi_{B}^{\prime} \varepsilon_{y}\left(p Y_{0}^{\prime}+q_{y}\right)\left(\left(Q_{b}+\varepsilon+p Y_{0}+q\right)^{4}-Q^{4}\right)\right|+\left|\int q_{y} q^{4} \varepsilon \psi_{B}^{\prime}\right| \\
& +\left|\int_{y<-\frac{B}{2}} \psi_{B}^{\prime} \varepsilon_{y}^{2}\left(Q_{b}+\varepsilon+p Y_{0}+q\right)^{4}\right| \\
& \lesssim\left(e^{-\frac{1}{2} B}+\delta\left(s_{0}^{-1}\right)+\delta\left(\alpha^{*}\right)\right) \int_{y<-\frac{B}{2}} \varphi_{i, B}^{\prime}\left(\varepsilon_{y}^{2}+\varepsilon^{2}\right)+\frac{1}{s^{4}} \\
& \leq \frac{1}{100} \int_{y<-B / 2}\left(\varepsilon_{y}^{2}+\varepsilon^{2}\right) \varphi_{i, B}^{\prime}+\frac{C}{s^{4}} .
\end{aligned}
$$


The remaining nonlinear term is estimated using the local $H^{2}$ control provided by localization (see more details in [38])

$$
\begin{aligned}
& \left|\int_{y<-\frac{B}{2}} \psi_{B}^{\prime}\left\{\left[-\varepsilon_{y y}+\varepsilon-Z\right]^{2}-\left[-\varepsilon_{y y}+\varepsilon\right]^{2}\right\}\right| \\
= & \left|\int_{y<-\frac{B}{2}} \psi_{B}^{\prime}\left(-2 \varepsilon_{y y}+2 \varepsilon-Z\right) Z\right| \\
\leq & \frac{1}{100} \int_{y<-\frac{B}{2}} \psi_{B}^{\prime}\left(\left|\varepsilon_{y y}\right|^{2}+|\varepsilon|^{2}\right)+C \int_{y<-\frac{B}{2}}\left(\varphi_{i, B}^{\prime}\right)^{2} Z^{2} \\
\leq & \frac{1}{100} \int_{y<-B / 2}\left[\varepsilon_{y y}^{2} \psi_{B}^{\prime}+\left(\varepsilon_{y}^{2}+\varepsilon^{2}\right) \varphi_{i, B}^{\prime}\right]+\frac{1}{s^{4}} .
\end{aligned}
$$

- In the region $y>\frac{B}{2}$, we have $\psi_{B}(y)=1$, so that several terms cancel in $f_{1,1}^{(i)}$. For the remainding terms, we argue as before. We rely on (B.9) to estimate:

$$
\int_{y>B / 2} \varepsilon^{2}\left|\varphi_{i, B}^{\prime \prime \prime}\right| \lesssim \frac{1}{B^{2}} \int_{y>B / 2} \varepsilon^{2} \varphi_{i, B}^{\prime} \leq \frac{1}{100} \int_{y>B / 2} \varepsilon^{2} \varphi_{i, B}^{\prime},
$$

and we use the exponential localization of $Q_{b}$ to the right and (B.13), to control:

$$
\begin{aligned}
& \left|\int_{y>B / 2}\left(\frac{\left(\varepsilon+Q_{b}+p Y_{0}+q\right)^{6}}{6}-\frac{\left(Q_{b}+p Y_{0}+q\right)^{6}}{6}-Q_{b}^{5} \varepsilon-5 Q^{4}\left(p Y_{0}+q\right)-Z \varepsilon\right) \varphi_{i, B}^{\prime}\right| \\
& \lesssim \int_{y>B / 2}\left(\varepsilon^{6}+\left(\left|Q_{b}\right|^{4}+p^{4}+q^{4}\right) \varepsilon^{2}+(|b|+|q|+|p|)^{2}|\varepsilon|\right) \varphi_{i, B}^{\prime} \\
& \lesssim\left(\delta\left(\alpha^{*}\right)+\delta\left(s_{0}^{-1}\right)+e^{-\frac{B}{10}}\right) \int_{y>B / 2} \varepsilon^{2} \varphi_{i, B}^{\prime}+\frac{1}{s^{4}} \\
& \leq \frac{1}{100} \int_{y>B / 2} \varepsilon^{2} \varphi_{i, B}^{\prime}+\frac{C}{s^{4}}, \\
& \mid \int_{y>B / 2}\left[\left(\varepsilon+Q_{b}+p Y_{0}+q\right)^{5}-\left(Q_{b}+p Y_{0}+q\right)^{5}-5 Q_{b}^{4} \varepsilon\right]\left(Q_{b}\right)_{y}\left(\psi_{B}-\varphi_{i, B}\right) \\
& -20 \int_{y>B / 2} Q^{\prime} Q^{3}\left(p Y_{0}+q\right) \varepsilon\left(\psi_{B}-\varphi_{i, B}\right) \mid \\
& \leq \frac{1}{100} \int_{y>B / 2} \varepsilon^{2} \varphi_{i, B}^{\prime}+\frac{C}{s^{4}} .
\end{aligned}
$$

Since $Y_{0} \in \mathcal{Y}$, we argue similarly to obtain

$$
\begin{aligned}
& \left|\int\left(\psi_{B}-\varphi_{i, B}\right) p Y_{0}^{\prime}\left[\left(Q_{b}+\varepsilon+p Y_{0}+q\right)^{5}-\left(Q_{b}+p Y_{0}+q\right)^{5}-5 Q^{4} \varepsilon\right]\right| \\
& \leq \frac{1}{100} \int_{y>B / 2} \varepsilon^{2} \varphi_{i, B}^{\prime}+\frac{C}{s^{4}} .
\end{aligned}
$$


Next, we have from (B.14),

$$
\begin{aligned}
& \left|\int\left(\varphi_{i, B}-\psi_{B}\right) q_{y}\left[\left(Q_{b}+\varepsilon+p Y_{0}+q\right)^{5}-\left(Q_{b}+p Y_{0}+q\right)^{5}-5 Q^{4} \varepsilon\right]\right| \\
& \leq \frac{1}{100} \int_{y>B / 2} \varepsilon^{2} \varphi_{i, B}^{\prime}+\frac{C}{s^{4}} .
\end{aligned}
$$

Also,

$$
\left|\int Z_{y} \varepsilon_{y} \psi_{B}^{\prime}\right|=\left|\int Z\left(\varepsilon_{y y} \psi_{B}^{\prime}+\varepsilon_{y} \psi_{B}^{\prime \prime}\right)\right| \leq \frac{1}{100} \int\left(\varepsilon_{y y}^{2}+\varepsilon_{y}^{2}+\varepsilon^{2}\right) \psi_{B}^{\prime}+\frac{C}{s^{4}} .
$$

- In the region $|y|<B / 2, \varphi_{i, B}(s, y)=1+y / B$ and $\psi_{B}(y)=1$. In particular, $\varphi_{i, B}^{\prime \prime \prime}=\psi_{B}^{\prime}=0$ in this region, and we obtain

$$
\begin{aligned}
\left(f_{1,1}^{(i)}\right)^{\sim}= & -\frac{1}{B} \int_{|y|<B / 2}\left\{3 \varepsilon_{y}^{2}+\varepsilon^{2}\right. \\
& +\frac{1}{3}\left(\left(\varepsilon+Q_{b}+p Y_{0}+q\right)^{6}-\left(Q_{b}+p Y_{0}+q\right)^{6}-6 Q_{b}^{5} \varepsilon\right. \\
& \left.\quad-30 Q^{4}\left(p Y_{0}+q\right) \varepsilon-6 Z \varepsilon\right) \\
& +2\left(\left(\varepsilon+Q_{b}+p Y_{0}+q\right)^{5}-\left(Q_{b}+p Y_{0}+q\right)^{5}-5 Q_{b}^{4} \varepsilon\right) y\left(Q_{b}\right)_{y} \\
& -40 y Q^{\prime} Q^{3}\left(p Y_{0}+q\right) \varepsilon \\
& \left.+2 y\left(p Y_{0}^{\prime}+q_{y}\right)\left(\left(\varepsilon+Q_{b}+p Y_{0}+q\right)^{5}-\left(Q_{b}+p Y_{0}+q\right)^{5}-5 Q_{b}^{4} \varepsilon\right)\right\} \\
= & -\frac{1}{B} \int_{|y|<B / 2}\left\{3 \varepsilon_{y}^{2}+\varepsilon^{2}-5 Q^{4} \varepsilon^{2}+20 y Q^{\prime} Q^{3} \varepsilon^{2}\right\}+R_{\mathrm{Vir}}(\varepsilon),
\end{aligned}
$$

where

$$
\begin{aligned}
R_{\mathrm{Vir}}(\varepsilon)= & -\frac{1}{B} \int_{|y|<B / 2}\left\{\frac { 1 } { 3 } \left(\left(\varepsilon+Q_{b}+p Y_{0}+q\right)^{6}-\left(Q_{b}+p Y_{0}+q\right)^{6}-6 Q_{b}^{5} \varepsilon\right.\right. \\
& \left.-30 Q^{4}\left(p Y_{0}+q\right) \varepsilon-6 Z \varepsilon-15 Q^{4} \varepsilon^{2}\right) \\
& +2\left(\left(\varepsilon+Q_{b}+p Y_{0}+q\right)^{5}-\left(Q_{b}+p Y_{0}+q\right)^{5}-5 Q_{b}^{4} \varepsilon\right) y\left(Q_{b}\right)_{y} \\
& -20 y Q^{\prime} Q^{3}\left(p Y_{0}+q\right) \varepsilon-10 y Q^{\prime} Q^{3} \varepsilon^{2} \\
& \left.+2 y\left(p Y_{0}^{\prime}+q_{y}\right)\left(\left(\varepsilon+Q_{b}+p Y_{0}+q\right)^{5}-\left(Q_{b}+p Y_{0}+q\right)^{5}-5 Q_{b}^{4} \varepsilon\right)\right\} .
\end{aligned}
$$


As before, we estimate

$$
\begin{aligned}
\left|R_{\text {Vir }}(\varepsilon)\right| & \lesssim \int_{|y|<B} \varepsilon^{2}\left(\delta\left(\alpha^{*}\right)+|b|+|p|+|q|\right)+\int_{|y|<B}|\varepsilon|\left(p^{2}+q^{2}+b^{2}\right) \\
& \lesssim \frac{1}{100} \int_{|y|<B / 2}\left(\varepsilon_{y}^{2}+\varepsilon^{2}\right)+\frac{1}{s^{4}} .
\end{aligned}
$$

We now recall from [38] the following coercivity result:

Lemma B.1 (Localized viriel estimate). There exists $B_{0}>100$ and $\mu_{3}>0$ such that if $B \geq B_{0}$, then

$$
\int_{|y|<B / 2}\left(3 \varepsilon_{y}^{2}+\varepsilon^{2}-5 Q^{4} \varepsilon^{2}+20 y Q^{\prime} Q^{3} \varepsilon^{2}\right) \geq \mu_{3} \int_{|y|<B / 2}\left(\varepsilon_{y}^{2}+\varepsilon^{2}\right)-\frac{1}{B} \int \varepsilon^{2} e^{-\frac{|y|}{2}} .
$$

Thus for $\alpha^{*}$ small enough we have

$$
\left(f_{1,1}^{(i)}\right)^{\sim} \leq-\frac{\mu_{3}}{2 B} \int_{|y|<B / 2}\left(\varepsilon_{y}^{2}+\varepsilon^{2}\right)+\frac{1}{B^{2}} \int \varepsilon^{2} e^{-\frac{|y|}{2}}+\frac{1}{s^{4}} .
$$

The collection of above estimates yields the bound

$$
f_{1,1}^{(i)} \leq-\frac{\mu_{4}}{B} \int\left[\psi_{B}^{\prime} \varepsilon_{y y}^{2}+\varphi_{i, B}^{\prime}\left(\varepsilon_{y}^{2}+\varepsilon^{2}\right)\right]+\frac{C}{s^{4}}
$$

for some universal $\mu_{4}>0$ independent of $B$.

Term $f_{1,2}^{(i)}$ : We decompose $f_{1,2}$ in a suitable way:

$$
\begin{aligned}
f_{1,2}^{(i)=} & 2\left(\frac{\lambda_{s}}{\lambda}+b\right) \int \Lambda Q(L \varepsilon)-2\left(\frac{\lambda_{s}}{\lambda}+b\right) \int \varepsilon\left(1-\varphi_{i, B}\right) \Lambda Q \\
& +2 b\left(\frac{\lambda_{s}}{\lambda}+b\right) \int \Lambda\left(\chi_{b} P\right)\left(-\left(\psi_{B} \varepsilon_{y}\right)_{y}+\varepsilon \varphi_{i, B}-\psi_{B} Z\right) \\
& +2\left(\frac{\lambda_{s}}{\lambda}+b\right) \int \Lambda Q\left(-\left(\psi_{B}\right)_{y} \varepsilon_{y}-\left(1-\psi_{B}\right) \varepsilon_{y y}+\left(1-\psi_{B}\right) Z\right) \\
& +2\left(\frac{\lambda_{s}}{\lambda}+b\right) \int \Lambda Q\left(Z-5 Q^{4} \varepsilon\right) .
\end{aligned}
$$

Observe from (2.25) that

$$
\int \Lambda Q(L \varepsilon)=(\varepsilon, L \Lambda Q)=-2(\varepsilon, Q)=0 .
$$

We now use the orthogonality conditions $(\varepsilon, y \Lambda Q)=0$ and the definition of $\varphi_{i, B}$ to estimate

$$
\left|\int \Lambda Q \varepsilon\left(1-\varphi_{i, B}\right)\right|=\left|\int \Lambda Q \varepsilon\left(1-\varphi_{i, B}+\frac{y}{B}\right)\right| \lesssim e^{-\frac{B}{8}} \mathcal{N}_{i, \mathrm{loc}}^{\frac{1}{2}},
$$


so that for $B$ large enough we have

$$
\begin{aligned}
\left|\left(\frac{\lambda_{s}}{\lambda}+b\right) \int \Lambda Q \varepsilon\left(1-\varphi_{i, B}\right)\right| & \lesssim\left(\mathcal{N}_{i, \mathrm{loc}}^{\frac{1}{2}}+\frac{1}{s^{2}}\right) e^{-\frac{B}{8}} \mathcal{N}_{i, \mathrm{loc}}^{\frac{1}{2}} \\
& \leq \frac{1}{500} \frac{\mu_{4}}{B} \mathcal{N}_{i, \mathrm{loc}}+\frac{C}{s^{4}}
\end{aligned}
$$

For the next term in $f_{1,2}^{(i)}$, we first integrate by parts to remove all derivatives on $\varepsilon$. Then, by the properties of $\varphi_{i, B}, \psi_{B}, P$ and $\chi_{b}$ (2.6), we obtain for $\alpha^{*}$ small,

$$
\begin{aligned}
& || 2 b\left(\frac{\lambda_{s}}{\lambda}+b\right) \int \Lambda\left(\chi_{b} P\right)\left(-\left(\psi_{B} \varepsilon_{y}\right)_{y}+\varepsilon \varphi_{i, B}-\psi_{B} Z\right) \mid \\
& \lesssim|b|\left(\mathcal{N}_{i, \mathrm{loc}}^{\frac{1}{2}}+\frac{1}{s^{2}}\right)^{2} \leq \frac{1}{500} \frac{\mu_{4}}{B} \mathcal{N}_{i, \mathrm{loc}}(s)+\frac{C}{s^{4}} .
\end{aligned}
$$

Next, integrating by parts, using the exponential decay of $Q$ and since $\psi_{B}(y) \equiv 1$ on $\left[-\frac{B}{2}, \infty\right)$ :

$$
\begin{aligned}
& \left|\left(\frac{\lambda_{s}}{\lambda}+b\right) \int \Lambda Q\left(-\left(\psi_{B}\right)_{y} \varepsilon_{y}-\left(1-\psi_{B}\right) \varepsilon_{y y}+\left(1-\psi_{B}\right) Z\right)\right| \\
\lesssim & \left(\mathcal{N}_{i, \mathrm{loc}}^{\frac{1}{2}}+\frac{1}{s^{2}}\right)^{2} \leq \frac{1}{500} \frac{\mu_{4}}{B} \mathcal{N}_{i, \mathrm{loc}}+\frac{C}{s^{4}},
\end{aligned}
$$

and finally

$$
\begin{aligned}
& \left|\left(\frac{\lambda_{s}}{\lambda}+b\right) \int \Lambda Q\left[Z-5 Q^{4} \varepsilon\right]\right| \lesssim\left(\mathcal{N}_{i, \mathrm{loc}}^{\frac{1}{2}}+\frac{1}{s^{2}}\right)\left(\mathcal{N}_{i, \mathrm{loc}}+\frac{1}{s^{2}}\right) \\
& \leq \frac{1}{500} \frac{\mu_{4}}{B} \mathcal{N}_{i, \mathrm{loc}}(s)+\frac{C}{s^{4}} .
\end{aligned}
$$

The collection of above estimates yields the bound

$$
\left|f_{1,2}^{(i)}\right| \leq \frac{1}{100} \frac{\mu_{4}}{B} \mathcal{N}_{i, \mathrm{loc}}+\frac{C}{s^{4}}
$$


$\operatorname{Term} f_{1,3}^{(i)}$ : Integrating by parts, we claim the identity

$$
\begin{aligned}
& -\frac{1}{6} \int \psi_{B}^{\prime}\left[\left(Q_{b}+p Y_{0}+q+\varepsilon\right)^{6}-Q_{b}^{6}-6 Q_{b}^{5} \varepsilon-6 Q^{5}\left(p Y_{0}+q\right)-6 Q_{b} q^{5}\right] \\
& =\int \psi_{B}\left(Q_{b}\right)_{y} Z-5 \int \psi_{B} Q^{\prime} Q^{4} \varepsilon+\int\left(Q_{b}^{5}-Q^{5}\right)_{y} \varepsilon \psi_{B} \\
& +5 \int\left(Q_{b}-Q\right)_{y} Q^{4}\left(p Y_{0}+q\right) \psi_{B}+\int \psi_{B} p Y_{0}^{\prime} Z+\int\left(Q^{5}-Q_{b}^{5}\right) p Y_{0}^{\prime} \psi_{B} \\
& +5 \int Q^{4}\left(p Y_{0}+q\right) p Y_{0}^{\prime} \psi_{B}+\int \psi_{B} p Y_{0}^{\prime} q^{5} Q_{b} \\
& +\int \psi_{B} q_{y}\left[\left(Q_{b}+p Y_{0}+q+\varepsilon\right)^{5}-Q^{5}\right]+\int \psi_{B} \varepsilon_{y} Z \\
& +5 \int \psi_{B} \varepsilon_{y} Q^{4}\left(p Y_{0}+q\right) .
\end{aligned}
$$

Therefore,

$$
\begin{aligned}
f_{1,3}^{(i)}= & \left(\frac{x_{s}}{\lambda}-1\right)\left\{2 \int Q^{\prime}\left[L \varepsilon-\psi_{B}^{\prime} \varepsilon_{y}+\left(1-\psi_{B}\right) \varepsilon_{y y}-\varepsilon\left(1-\varphi_{i, B}\right)\right]\right. \\
& +2 \int\left(Q_{b}-Q+\varepsilon+p Y_{0}\right)_{y}\left[-\psi_{B}^{\prime} \varepsilon_{y}-\psi_{B} \varepsilon_{y y}+\varepsilon \varphi_{i, B}\right] \\
& +\frac{1}{3} \int \psi_{B}^{\prime}\left[\left(Q_{b}+p Y_{0}+q+\varepsilon\right)^{6}-Q_{b}^{6}-6 Q_{b}^{5} \varepsilon-6 Q^{5}\left(p Y_{0}+q\right)-6 Q_{b} q^{5}\right] \\
& +2 \int \varepsilon \psi_{B}\left(Q_{b}^{5}-Q^{5}\right)_{y}+10 \int \psi_{B}\left(Q_{b}-Q\right)_{y} Q^{4}\left(p Y_{0}+q\right) \\
& +2 \int \psi_{B}\left(Q^{5}-Q_{b}^{5}\right) p Y_{0}^{\prime}+10 \int Q^{4}\left(p Y_{0}+q\right) p Y_{0}^{\prime} \psi_{B}+2 \int \psi_{B} p Y_{0}^{\prime} q^{5} Q_{b} \\
& \left.+2 \int \psi_{B} q_{y}\left[\left(Q_{b}+p Y_{0}+q+\varepsilon\right)^{5}-Q^{5}-5 Q_{b} q^{4}\right]+10 \int \psi_{B} \varepsilon_{y} Q^{4}\left(p Y_{0}+q\right)\right\} .
\end{aligned}
$$

The first term is treated using the cancellation $L Q^{\prime}=0$ and the orthogonality conditions $(\varepsilon, \Lambda Q)=(\varepsilon, Q)=0$, so that $\left(y Q^{\prime}, \varepsilon\right)=0$. Thus, by the definitions of $\varphi_{i, B}$ and $\psi_{B}$,

$$
\begin{aligned}
& \left|2\left(\frac{x_{s}}{\lambda}-1\right) \int Q^{\prime}\left[L \varepsilon-\psi_{B}^{\prime} \varepsilon_{y}+\left(1-\psi_{B}\right) \varepsilon_{y y}-\varepsilon\left(1-\varphi_{i, B}\right)\right]\right| \\
& =\left|2\left(\frac{x_{s}}{\lambda}-1\right) \int Q^{\prime}\left[-\psi_{B}^{\prime} \varepsilon_{y}+\left(1-\psi_{B}\right) \varepsilon_{y y}-\varepsilon\left(1+\frac{y}{B}-\varphi_{i, B}\right)\right]\right| \\
& \lesssim\left(\mathcal{N}_{i, \mathrm{loc}}^{\frac{1}{2}}+\frac{1}{s^{2}}\right) e^{-\frac{B}{10}} \mathcal{N}_{i, \mathrm{loc}}^{\frac{1}{2}} \leq \frac{1}{500} \frac{\mu_{4}}{B} \mathcal{N}_{i, \mathrm{loc}}+\frac{C}{s^{4}} .
\end{aligned}
$$


Then, as before, integrating by parts, and using the Cauchy-Schwarz inequality,

$$
\begin{aligned}
& \quad\left|2 b\left(\frac{x_{s}}{\lambda}-1\right) \int\left(\chi_{b} P\right)_{y}\left[-\psi_{B}^{\prime} \varepsilon_{y}-\psi_{B} \varepsilon_{y y}+\varepsilon \varphi_{i, B}\right]\right| \\
& \lesssim \frac{1}{s}\left(\mathcal{N}_{i, \mathrm{loc}}^{\frac{1}{2}}+\frac{1}{s^{2}}\right) B^{\frac{1}{2}} \mathcal{N}_{i, \mathrm{loc}} \leq \frac{1}{500} \frac{\mu_{4}}{B} \mathcal{N}_{i, \mathrm{loc}}+\frac{C}{s^{4}}, \\
& \left|2\left(\frac{x_{s}}{\lambda}-1\right) \int \varepsilon_{y}\left[-\psi_{B}^{\prime} \varepsilon_{y}-\psi_{B} \varepsilon_{y y}+\varepsilon \varphi_{i, B}\right]\right| \\
& \lesssim \delta\left(\alpha^{*}\right) \int\left(\varepsilon_{y}^{2}+\varepsilon^{2}\right) \varphi_{i, B}^{\prime} \leq \frac{1}{500} \frac{\mu_{4}}{B} \int\left(\varepsilon_{y}^{2}+\varepsilon^{2}\right) \varphi_{i, B}^{\prime}+\frac{C}{s^{4}}, \\
& \left|\frac{x_{s}}{\lambda}-1\right|\left|\int p Y_{0}^{\prime}\left[-\psi_{B}^{\prime} \varepsilon_{y}-\psi_{B} \varepsilon_{y y}+\varepsilon \varphi_{i, B}\right]\right| \\
& \lesssim\left(\mathcal{N}_{i, \mathrm{loc}}^{\frac{1}{2}}+\frac{1}{s^{2}}\right) \frac{1}{s} \mathcal{N}_{i, \mathrm{loc}}^{\frac{1}{2}} \leq \frac{1}{500} \frac{\mu_{4}}{B} \int\left(\varepsilon_{y}^{2}+\varepsilon^{2}\right) \varphi_{i, B}^{\prime}+\frac{C}{s^{4}} .
\end{aligned}
$$

In conclusion, for $f_{1,3}^{(i)}$ one gets

$$
\left|f_{1,3}^{(i)}\right| \leq \frac{1}{50} \frac{\mu_{4}}{B} \int\left(\varepsilon_{y}^{2}+\varepsilon^{2}\right) \varphi_{i, B}^{\prime}+\frac{C}{s^{4}},
$$

for $B$ large enough and $\alpha^{*}$ small enough, $s_{0}$ large enough.

$\operatorname{Term} f_{1,4}^{(i)}$ : Recall

$$
f_{1,4}^{(i)}=-2 b_{s} \int\left(\chi_{b}+\gamma y\left(\chi_{b}\right)_{y}\right) P\left(-\psi_{B} \varepsilon_{y y}-\psi_{B}^{\prime} \varepsilon_{y}+\varepsilon \varphi_{i, B}-\psi_{B} Z\right) .
$$

We estimate after integrations by parts

$$
\begin{aligned}
&\left|\int\left(\chi_{b}+\gamma y\left(\chi_{b}\right)_{y}\right) P\left(-\psi_{B} \varepsilon_{y}\right)_{y}\right| \lesssim \int|\varepsilon|\left|\left(\psi_{B}\left(\left(\chi_{b}+\gamma y\left(\chi_{b}\right)_{y}\right) P\right)_{y}\right)_{y}\right| \lesssim B^{\frac{1}{2}} \mathcal{N}_{i, \mathrm{loc}}^{\frac{1}{2}}, \\
&\left|\int\left(\chi_{b}+\gamma y\left(\chi_{b}\right)_{y}\right) P \varepsilon \varphi_{i, B}\right| \lesssim B^{\frac{1}{2}} \mathcal{N}_{i, \mathrm{loc}}^{\frac{1}{2}} .
\end{aligned}
$$

The estimate of the nonlinear term follows from (B.13) and $\psi \leq\left(\varphi_{i}^{\prime}\right)^{2}$ for $y<-\frac{1}{2}$ :

$$
\begin{aligned}
\left|\int\left(\chi_{b}+\gamma y\left(\chi_{b}\right)_{y}\right) P \psi_{B} Z\right| & \lesssim \int \psi_{B}\left(\left.\left(\left|Q_{b}\right|^{4} \mid+p^{4}+q^{4}\right) \varepsilon|+| \varepsilon\right|^{5}+b^{2}+p^{2}+q^{2}\right) \\
& \lesssim B^{\frac{1}{2}}\left(\int\left(|\varepsilon|^{2}+|\varepsilon|^{6}\right) \psi_{B}\right)^{\frac{1}{2}}+\frac{1}{s^{2}} \\
& \lesssim B^{\frac{1}{2}}\left(\int\left(\varepsilon_{y}^{2}+\varepsilon^{2}\right) \varphi_{i, B}^{\prime}\right)^{\frac{1}{2}}+\frac{1}{s^{2}} .
\end{aligned}
$$


Together with (3.14), these estimates yield the bound

$$
\left|f_{1,4}\right| \leq \frac{1}{500} \frac{\mu_{4}}{B} \int\left(\varepsilon_{y}^{2}+\varepsilon^{2}\right) \varphi_{i, B}^{\prime}+\frac{C}{s^{4}} .
$$

$\operatorname{Term} f_{1,5}^{(i)}$ : Recall:

$$
f_{1,5}^{(i)}=2 \int \Psi_{b}\left(-\left(\psi_{B} \varepsilon_{y}\right)_{y}+\varepsilon \varphi_{i, B}-\psi_{B} Z\right) .
$$

We now rely on (3.15) to estimate by integration by parts and the Cauchy-Schwarz inequality,

$$
\left|\int\left(\Psi_{b}\right)_{y} \psi_{B} \varepsilon_{y}\right| \lesssim B^{\frac{1}{2}} b^{2} \mathcal{N}_{i, \text { loc }}^{\frac{1}{2}} \leq \frac{1}{500} \frac{\mu_{4}}{B} \mathcal{N}_{i, \text { loc }}+\frac{C}{s^{4}} .
$$

By (2.10) and the exponential decay of $\varphi_{i, B}$ in the left,

$$
\left|\int \Psi_{b} \varphi_{i, B} \varepsilon\right| \lesssim\left(b^{2} B^{\frac{1}{2}}+e^{-\frac{1}{\left.2|b|\right|^{Y}}}\right)|b|^{1+\gamma} \mathcal{N}_{i, \text { loc }}^{\frac{1}{2}} \leq \frac{1}{500} \frac{\mu_{4}}{B} \mathcal{N}_{i, \text { loc }}+C|b|^{4} .
$$

For the nonlinear term, similarly and using (B.13),

$$
\left|\int \Psi_{b} \psi_{B} Z\right| \leq \frac{1}{500} \frac{\mu_{4}}{B} \int\left(\varepsilon_{y}^{2}+\varepsilon^{2}\right) \varphi_{i, B}^{\prime}+\frac{C}{s^{4}} .
$$

The collection of above estimates yields the bound

$$
\left|f_{1,5}^{(i)}\right| \leq \frac{1}{100} \frac{\mu_{4}}{B} \int\left(\varepsilon_{y}^{2}+\varepsilon^{2}\right) \varphi_{i, B}^{\prime}+\frac{C}{s^{4}} .
$$

$\operatorname{Term} f_{1,6}^{(i)}$ : Recall that this term writes

$$
\left|\int\left(-p_{s} Y_{0}+\left(5 Q^{4}(p-q)\right)_{y}+\frac{\lambda_{s}}{\lambda} p \Lambda Y_{0}\right)\left(-\left(\psi_{B} \varepsilon_{y}\right)_{y}+\varepsilon \varphi_{i, B}-\psi_{B} Z\right)\right| .
$$

By (3.15), (2.42), (3.14),

$$
\begin{aligned}
& \left|\left(-p_{s} Y_{0}+\left(5 Q^{4}(p-q)\right)_{y}+\frac{\lambda_{s}}{\lambda} p \Lambda Y_{0}\right)\right|+\left|\left(-p_{s} Y_{0}+\left(5 Q^{4}(p-q)\right)_{y}+\frac{\lambda_{s}}{\lambda} p \Lambda Y_{0}\right)_{y}\right| \\
& \lesssim e^{-\frac{|y|}{2}}\left(\frac{1}{s^{2}}+\int \varepsilon^{2} e^{-\frac{|y|}{2}}\right),
\end{aligned}
$$

and thus $\left|f_{1,6}^{(i)}\right| \lesssim \delta\left(\alpha^{*}\right) \int \varepsilon^{2} e^{-\frac{|y|}{2}}+\frac{1}{s^{4}}$. 
Step 4. $f_{2}^{(i, j)}$ term. Recall:

$$
f_{2}^{(i, j)}=2 \frac{\lambda_{s}}{\lambda} \int \Lambda \varepsilon\left(-\left(\psi_{B} \varepsilon_{y}\right)_{y}+\varepsilon \varphi_{i, B}-\psi_{B} Z\right)+\frac{j}{s} \mathcal{F}_{i}
$$

We integrate by parts to compute:

$$
\begin{aligned}
\int \Lambda \varepsilon\left(\psi_{B} \varepsilon_{y}\right)_{y} & =-\int \varepsilon_{y}^{2} \psi_{B}+\frac{1}{2} \int \varepsilon_{y}^{2} y \psi_{B}^{\prime}, \\
\int(\Lambda \varepsilon) \varepsilon \varphi_{i, B} & =-\frac{1}{2} \int \varepsilon^{2} y \varphi_{i, B}^{\prime},
\end{aligned}
$$

$$
\begin{aligned}
& \int \Lambda \varepsilon \psi_{B} Z \\
& =\int\left(\frac{\varepsilon}{2}+y \varepsilon_{y}\right) \psi_{B}\left[\left(Q_{b}+p Y_{0}+q+\varepsilon\right)^{5}-Q_{b}^{5}-5 Q^{4}\left(p Y_{0}+q\right)-q^{5}\right] \\
& =\int \frac{\varepsilon}{2} \psi_{B}\left[\left(Q_{b}+p Y_{0}+q+\varepsilon\right)^{5}-Q_{b}^{5}-5 Q^{4}\left(p Y_{0}+q\right)-q^{5}\right] \\
& -\int\left(y \psi_{B}\right)_{y}\left[\frac{\left(Q_{b}+p Y_{0}+q+\varepsilon\right)^{6}}{6}-\frac{\left(Q_{b}+p Y_{0}+q\right)^{6}}{6}-Q_{b}^{5} \varepsilon\right. \\
& \left.-5 Q^{4}\left(p Y_{0}+q\right) \varepsilon-Q_{b}^{5}\left(p Y_{0}+q\right)-q^{5} \varepsilon\right] \\
& -\int y \psi_{B}\left(Q_{b}\right)_{y}\left[\left(Q_{b}+p Y_{0}+q+\varepsilon\right)^{5}-\left(Q_{b}+p Y_{0}+q\right)^{5}-5 Q_{b}^{4} \varepsilon\right. \\
& \left.-5 Q_{b}^{4}\left(p Y_{0}+q\right) \varepsilon-20 Q_{b}^{3}\left(p Y_{0}+q\right) \varepsilon\right] \\
& -\int y \psi_{B} p Y_{0}^{\prime}\left[\left(Q_{b}+p Y_{0}+q+\varepsilon\right)^{5}-\left(Q_{b}+p Y_{0}+q\right)^{5}-5 Q^{4} \varepsilon\right] \\
& +5 \int\left(Q_{b}^{4}-Q^{4}\right)_{y}\left(p Y_{0}+q\right) \varepsilon y \psi_{B} \\
& -\int y \psi_{B}\left[\left(Q_{b}+p Y_{0}+q+\varepsilon\right)^{5}-\left(Q_{b}+p Y_{0}+q\right)^{5}-5 Q^{4} \varepsilon-Q_{b}^{5}-5 q^{4} \varepsilon\right] .
\end{aligned}
$$


Thus,

$$
\begin{aligned}
& f_{2}^{(i, j)}=2 \frac{\lambda_{s}}{\lambda}\left\{\int \varepsilon_{y}^{2} \psi_{B}-\frac{1}{2} \int \varepsilon_{y}^{2} y \psi_{B}^{\prime}-\frac{1}{2} \int \varepsilon^{2} y \varphi_{i, B}^{\prime}\right. \\
& +\int \frac{\varepsilon}{2} \psi_{B}\left[\left(Q_{b}+p Y_{0}+q+\varepsilon\right)^{5}-Q_{b}^{5}-5 Q^{4}\left(p Y_{0}+q\right)-q^{5}\right] \\
& -\int\left(y \psi_{B}\right)_{y}\left[\frac{\left(Q_{b}+p Y_{0}+q+\varepsilon\right)^{6}}{6}-\frac{\left(Q_{b}+p Y_{0}+q\right)^{6}}{6}-Q_{b}^{5} \varepsilon\right. \\
& \left.-5 Q^{4}\left(p Y_{0}+q\right) \varepsilon-Q_{b}^{5}\left(p Y_{0}+q\right)-q^{5} \varepsilon\right] \\
& -\int y \psi_{B}\left(Q_{b}\right)_{y}\left[\left(Q_{b}+p Y_{0}+q+\varepsilon\right)^{5}-\left(Q_{b}+p Y_{0}+q\right)^{5}-5 Q_{b}^{4} \varepsilon\right. \\
& \left.-5 Q_{b}^{4}\left(p Y_{0}+q\right) \varepsilon-20 Q_{b}^{3}\left(p Y_{0}+q\right) \varepsilon\right] \\
& -\int y \psi_{B} p Y_{0}^{\prime}\left[\left(Q_{b}+p Y_{0}+q+\varepsilon\right)^{5}-\left(Q_{b}+p Y_{0}+q\right)^{5}-5 Q^{4} \varepsilon\right] \\
& +5 \int\left(Q_{b}^{4}-Q^{4}\right)_{y}\left(p Y_{0}+q\right) \varepsilon y \psi_{B} \\
& \left.-\int y \psi_{B}\left[\left(Q_{b}+p Y_{0}+q+\varepsilon\right)^{5}-\left(Q_{b}+p Y_{0}+q\right)^{5}-5 Q^{4} \varepsilon-Q_{b}^{5}-5 q^{4} \varepsilon\right]\right\}+\frac{j}{s} \mathcal{F}_{i} .
\end{aligned}
$$

For $y<-B$, we use the exponential decay of $\psi_{B}, \varphi_{i, B}$ and (B.9) to estimate:

$$
\begin{aligned}
& \int_{y<-\frac{B}{2}}\left(\psi_{B}+|y| \psi_{B}^{\prime}+\varphi_{i, B}\right)\left(\varepsilon_{y}^{2}+\varepsilon^{2}\right)+|y| \varphi_{i, B}^{\prime} \varepsilon^{2} \\
& \lesssim \int_{y<-\frac{B}{2}} \varepsilon_{y}^{2} \varphi_{i, B}^{\prime}+\int_{y<-\frac{B}{2}}|y| \varphi_{i, B}^{\prime} \varepsilon^{2} \\
& \lesssim \int \varepsilon_{y}^{2} \varphi_{i, B}^{\prime}+\left(\int_{y<-\frac{B}{2}}|y|^{100} e^{\frac{y}{B}} \varepsilon^{2}\right)^{\frac{1}{100}}\left(\int_{y<-\frac{B}{2}} e^{\frac{y}{B}} \varepsilon^{2}\right)^{\frac{99}{100}} \\
& \lesssim \int \varepsilon_{y}^{2} \varphi_{i, B}^{\prime}+\mathcal{N}_{i, \mathrm{loc}}^{\frac{9}{10}},
\end{aligned}
$$

where we have used $\int_{y<-\frac{B}{2}}|y|^{100} e^{\frac{y}{B}} \varepsilon^{2} \leq\|\varepsilon\|_{L^{2}}^{2} \leq \delta\left(\alpha^{*}\right)$.

Together with similar estimates for the other terms, this yields the bound:

$$
\begin{aligned}
\left|\left(f_{2}^{(i, j)}\right)^{<}\right| & \lesssim\left(\frac{1}{s}+\mathcal{N}_{i, \mathrm{loc}}^{\frac{1}{2}}\right)\left(\int \varepsilon_{y}^{2} \varphi_{i, B}^{\prime}+\mathcal{N}_{i, \mathrm{loc}}^{\frac{9}{10}}+\frac{1}{s^{4}}\right) \\
& \lesssim \delta\left(\alpha^{*}\right) \int\left(\varepsilon_{y}^{2}+\varepsilon^{2}\right) \varphi_{i, B}^{\prime}+\frac{1}{s^{4}} .
\end{aligned}
$$

The middle term $f_{2}^{(i, j)}$ is also estimated as follows:

$$
\left|f_{2}^{(i, j)}\right| \leq \delta\left(\alpha^{*}\right) \int\left(\varepsilon_{y}^{2}+\varepsilon^{2}\right) \varphi_{i, B}^{\prime}+\frac{1}{s^{4}} .
$$


It only remains to estimate $\left(f_{2}^{(i, j)}\right)^{>}$. Most terms in $\left(f_{2}^{(i, j)}\right.$ are easily estimated similarly as before. We focus on the following two delicate terms (because of weight at $+\infty)$ :

$$
\int_{y>\frac{B}{2}} \varepsilon^{2} y \varphi_{i, B}^{\prime}, \quad \frac{j}{s} \int_{y>\frac{B}{2}} \varepsilon^{2} \varphi_{i, B} .
$$

The function $\psi_{B}$ being bounded, the other terms are easier.

First, using (B.3),

$$
\begin{aligned}
\frac{1}{s} \int_{y>\frac{B}{2}} \varepsilon^{2} \varphi_{i, B} & \lesssim \frac{1}{s}\left(1+\frac{1}{\lambda^{\frac{10}{9}}}\right) \mathcal{N}_{2, \text { loc }}^{\frac{8}{9}} \\
& \lesssim s^{\frac{10}{9} \beta-1} \mathcal{N}_{2, \mathrm{loc}}^{\frac{8}{9}} \leq \frac{1}{100} \frac{\mu_{4}}{B} \mathcal{N}_{2, \mathrm{loc}}+s^{10 \beta-9}
\end{aligned}
$$

Second, using $\mathcal{N}_{2, \text { loc }} \lesssim s^{-\frac{5}{2}}$ and $\beta<\frac{7}{8}$,

$$
\begin{aligned}
\int_{y>\frac{B}{2}} \varepsilon^{2} y \varphi_{i, B}^{\prime} & \lesssim\left(\frac{1}{s}+\left(\int \varepsilon^{2} e^{-\frac{|y|}{10}}\right)^{\frac{1}{2}}\right) \int_{y>\frac{B}{2}} \varepsilon^{2} \varphi_{i, B} \\
& \lesssim\left(\frac{1}{s}+\mathcal{N}_{2, \mathrm{loc}}^{\frac{1}{2}}\right) s^{\frac{10}{9} \beta} \mathcal{N}_{2, \mathrm{loc}}^{\frac{8}{9}} \leq \frac{1}{100} \frac{\mu_{4}}{B} \mathcal{N}_{2, \mathrm{loc}}+s^{10 \beta-9}
\end{aligned}
$$

Step 5. $f_{3}^{(i)}$ term.

$$
\begin{aligned}
f_{3}^{(i)}= & 2 \int \psi_{B}\left(Q_{b}\right)_{s}\left[\left(\varepsilon+Q_{b}+p Y_{0}+q\right)^{5}-\left(Q_{b}+p Y_{0}+q\right)^{5}-5 \varepsilon Q_{b}^{4}\right] \\
& -2 \int \psi_{B}\left(p_{s} Y_{0}+q_{s}\right)\left[\left(\varepsilon+Q_{b}+p Y_{0}+q\right)^{5}-\left(Q_{b}+p Y_{0}+q\right)^{5}-5 \varepsilon Q^{4}\right] \\
& +10 \int \psi_{B} q_{s} q^{4} \varepsilon .
\end{aligned}
$$

First,

$$
\begin{aligned}
& \left|\left(Q_{b}\right)_{s}\right|=\left|b_{s} P\left(\chi\left(|b|^{\gamma} y\right)+\gamma|b|^{\gamma} y \chi^{\prime}\left(|b|^{\gamma} y\right)\right)\right| \lesssim\left|b_{s}\right|, \\
& \left|\int \psi_{B}\left(Q_{b}\right)_{s}\left[\left(\varepsilon+Q_{b}+p Y_{0}+q\right)^{5}-\left(Q_{b}+p Y_{0}+q\right)^{5}-5 \varepsilon Q_{b}^{4}\right]\right| \\
& \lesssim\left|b_{s}\right| \int \psi_{B}\left(\varepsilon^{2}\left(\left|Q_{b}\right|^{3}+|p|^{3}+|q|^{3}+\delta\left(\alpha^{*}\right)\right)+|\varepsilon|\left(e^{-\frac{|y|}{2}} p^{4}+q^{4}\right)\right) \\
& \lesssim\left(\frac{1}{s^{2}}+\mathcal{N}_{i, \text { loc }}\right)\left(\int\left(\varepsilon_{y}^{2}+\varepsilon^{2}\right) \psi_{B}+\left(\int \varepsilon^{2} \psi_{B}\right)^{\frac{1}{2}}\left(\int q^{8} \psi_{B}\right)^{\frac{1}{2}}\right) \\
& \lesssim\left(\delta\left(\alpha^{*}\right)+\delta\left(s_{0}^{-1}\right)\right) \int \varepsilon^{2} \varphi_{i, B}^{\prime}+\frac{C}{s^{4}} .
\end{aligned}
$$


For the next two terms, we first remark that by explicit computations:

$$
\int \psi_{B}\left(p_{s} Y_{0}+q_{s}\right)^{2} \lesssim \frac{1}{s^{2}}
$$

Thus, as before,

$$
\begin{aligned}
& \left|\int \psi_{B}\left(p_{s} Y_{0}+q_{s}\right)\left[\left(\varepsilon+Q_{b}+p Y_{0}+q\right)^{5}-\left(Q_{b}+p Y_{0}+q\right)^{5}-5 \varepsilon Q^{4}\right]\right| \\
& \lesssim\left(\delta\left(\alpha^{*}\right)+\delta\left(s_{0}^{-1}\right)\right) \int \varepsilon^{2} \varphi_{i, B}^{\prime}+\frac{C}{s^{4}} .
\end{aligned}
$$

Finally,

$$
\left|\int \psi_{B} q_{s} q^{4} \varepsilon\right| \lesssim\left(\int \psi_{B}\left(q_{s} q^{4}\right)^{2}\right)^{\frac{1}{2}}\left(\int \psi_{B} \varepsilon^{2}\right)^{\frac{1}{2}} \lesssim \int \psi_{B} \varepsilon^{2}+\frac{1}{s^{4}}
$$

Step 6. Proof of (3.22). We proceed as in [38]. Recall that for $B$ large enough, $\mu>0$,

$$
\int \psi_{B} \varepsilon_{y}^{2}+\varphi_{i, B} \varepsilon^{2}-5 \psi_{B} Q^{4} \varepsilon^{2} \geq \mu \mathcal{N}_{i}
$$

We only have to estimate the error term as follows. For $s_{0}$ large enough, and $\alpha^{*}$ small enough,

$$
\begin{aligned}
& \left|\int\left(\left(Q_{b}+\varepsilon+p Y_{0}+q\right)^{6}-\left(Q_{b}+p Y_{0}+q\right)^{6}-6 \varepsilon\left(Q_{b}^{5}+q^{5}+5 Q^{4}\left(p Y_{0}+q\right)\right)-6 Q^{4} \varepsilon^{2}\right) \psi_{B}\right| \\
& \lesssim \frac{1}{s^{2}}\left(\int \psi_{B} \varepsilon^{2}\right)^{\frac{1}{2}}+\frac{1}{s} \int \psi_{B} \varepsilon^{2} \leq \frac{\mu}{10} \mathcal{N}_{i}+\frac{1}{s^{4}} .
\end{aligned}
$$

This concludes the proof of Lemma 3.4.

\section{References}

[1] T. Aubin, Équations différentielles non linéaires et problème de Yamabe concernant la courbure scalaire, J. Math. Pures Appl. 55 (1976), 269-296.

[2] J. Bourgain and W. WANG, Construction of blowup solutions for the nonlinear Schrödinger equation with critical nonlinearity, Ann. Scuola Norm. Sup. Pisa Cl. Sci. (4) 25 (1997), 197-215 (1998).

[3] R. CôTE, Construction of solutions to the $L^{2}$-critical KdV equation with a given asymptotic behaviour, Duke Math. J. 138 (2007), 487-531.

[4] R. Côte, Y. MARTEL and F. MERLE, Construction of multi-soliton solutions for the $L^{2}$. supercritical $g K d V$ and NLS equations, arXiv:0905.0470.

[5] R. CôTE and H. ZAAG, Construction of a multisoliton blowup solution to the semilinear wave equation in one space dimension, Commun. Pure Appl. Math. 66 (2013), 1541-1581. 
[6] R. DonNinger and J. KRIEGER, Nonscattering solutions and blowup at infinity for the critical wave equation, Math. Ann. 357 (2013), 89-163.

[7] T. DUYCKAERTS, C. KeNIG and F. MERLE, Universality of blow-up profile for small radial type II blow-up solutions of the energy-critical wave equation, J. Eur. Math. Soc. (JEMS) 13 (2011), 533-599.

[8] T. DuYCKAeRTS, C. Kenig and F. Merle, Universality of the blow-up profile for small type II blowup solutions of the energy-critical wave equation: the non radial case, J. Eur. Math. Soc. (JEMS) 14 (2012), 1389-1454.

[9] T. DUYCKAERTS and F. MERLE, Dynamics of threshold solutions for energy-critical wave equation, Int. Math. Res. Pap. IMRP 2007, Art. ID rpn002, 67 pp. (2008).

[10] T. DUYCKAERTS and F. MERLE, Dynamic of threshold solutions for energy-critical NLS, Geom. Funct. Anal. 18 (2009), 1787-1840.

[11] T. DUYCKAERTS and S. ROUDENKo, Threshold solutions for the focusing $3 D$ cubic Schrödinger equation, Rev. Mat. Iberoam. 26 (2010), 1-56.

[12] G. FiBICH, F. MERLE and P. RAPHAËL, Proof of a spectral property related to the singularity formation for the L2 critical nonlinear Schrödinger equation, Phys. D 220 (2006), $1-13$.

[13] M. Fila, J. R. King, M. WinKLER and E. YANAGIDA, Very slow grow-up of solutions of a semi-linear parabolic equation, Proc. Edinb. Math. Soc. (2) 54 (2011), 381-400.

[14] B. Gidas, W. M. Ni and L. NirenberG, Symmetry and related properties via the maximum principle, Comm. Math. Phys. 68 (1979), 209-243.

[15] J. GinIBRE and G. Velo, On a class of nonlinear Schrödinger equations. I. The Cauchy problem, general case, J. Funct. Anal. 32 (1979), 1-32.

[16] S. Gustafson, K. NAKANishi and T.-P. TsAi, Asymptotic stability, concentration and oscillations in harmonic map heat flow, Landau Lifschitz and Schrödinger maps on $\mathbb{R}^{2}$, Comm. Math. Phys. 300 (2010), 205-242.

[17] M. HillaiRET and P. RAPHAËL, Smooth type II blow-up solutions to the four-dimensional energy-critical wave equation, Anal. PDE 5 (2012), 777-829.

[18] T. KATO, On the Cauchy problem for the (generalized) Korteweg-de Vries equation, In: "Studies in Applied Mathematics", Adv. Math. Suppl. Stud., Vol. 8, Academic Press, New York, 1983, 93-128,

[19] C.E. KENIG and F. MERLE, Global well-posedness, scattering and blow-up for the energycritical, focusing, nonlinear Schrödinger equation in the radial case, Invent. Math. 166 (2006), 645-675.

[20] C. E. Kenig, G. PonCE and L. VeGA, Well-posedness and scattering results for the generalized Korteweg-de Vries equation via the contraction principle, Commun. Pure Appl. Math. 46, (1993), 527-620.

[21] C.E. Kenig, G. Ponce and L. VegA, On the concentration of blow-up solutions for the generalized KdV equation critical in $L^{2}$, In: "Nonlinear Wave Equations" (Providence, RI, 1998), Contemp. Math., Vol. 263, Amer. Math. Soc., Providence, RI, 2000, 131-156.

[22] C.E. KENIG and F. MERLE, Global well-posedness, scattering and blow-up for the energycritical, focusing, non-linear Schrödinger equation in the radial case, Invent. Math. 166 (2006), 645-675.

[23] R. Killip, S. Kwon, S. Shao and M. Visan, On the mass-critical generalized KdV equation, Discrete Contin. Dyn. Syst. 32 (2012), 191-221.

[24] J. KRIEGER, Y. MARTEL and P. RAPHAËL, Two-soliton solutions to the three-dimensional gravitational Hartree equation, Commun. Pure Appl. Math. 62 (2009), 1501-1550.

[25] J. Krieger, K. NAKANishi and W. Schlag, Global dynamics away from the ground state for the energy-critical nonlinear wave equation, Amer. J. Math. 135 (2013), 935-965.

[26] J. KRIEGER and W. SCHLAG, Non-generic blow-up solutions for the critical focusing NLS in 1-D, J. Eur. Math. Soc. (JEMS) 11 (2009), 1-125.

[27] J. KRIEgER, W. Schlag and D. TATARU, Renormalization and blow-up for charge one equivariant critical wave maps, Invent. Math. 171 (2008), 543-615. 
[28] J. KRIEGER, W. SCHLAG and D. TATARU, Slow blow-up solutions for the $H^{1}\left(\mathbb{R}^{3}\right)$ critical focusing semilinear wave equation, Duke Math. J. 147 (2009), 1-53.

[29] M. K. Kwong, Uniqueness of positive solutions of $\Delta u-u+u^{p}=0$ in $\mathbb{R}^{n}$, Arch. Ration. Mech. Anal. 105 (1989), 243-266.

[30] Y. MARTEL, Asymptotic N-soliton-like solutions of the subcritical and critical generalized Korteweg-de Vries equations, Amer. J. Math. 127 (2005), 1103-1140.

[31] Y. MARTEL and F. MERLE, A Liouville theorem for the critical generalized Korteweg-de Vries equation, J. Math. Pures Appl. 79 (2000), 339-425.

[32] Y. MARTEL and F. MERLE, Instability of solitons for the critical generalized Korteweg-de Vries equation, Geom. Funct. Anal. 11 (2001), 74-123.

[33] Y. MARTEL and F. Merle, Stability of blow-up profile and lower bounds for blow-up rate for the critical generalized KdV equation, Ann. of Math. 155 (2002), 235-280.

[34] Y. MARTEL and F. Merle, Blow up in finite time and dynamics of blow-up solutions for the $L^{2}$-critical generalized KdV equation, J. Amer. Math. Soc. 15 (2002), 617-664.

[35] Y. MARTEL and F. Merle, Nonexistence of blow-up solution with minimal $L^{2}$-mass for the critical gKdV equation, Duke Math. J. 115 (2002), 385-408.

[36] Y. MARTEL and F. MERLE, Multi solitary waves for nonlinear Schrödinger equations, Ann. Inst. H. Poincaré Anal. Non Linéaire 23 (2006), 849-864.

[37] Y. Martel and F. Merle, Description of two solitons collision for the quartic gKdV equation, Ann. of Math. 174 (2011), 757-857.

[38] Y. MARTEL, F. MERLE and P. RAPHAËL, Blow up for the critical generalized Korteweg-de Vries equation I: Dynamics near the soliton, Acta Math. 212 (2014), 59-140.

[39] Y. MARTEL, F. MERLE and P. RAPHAËL, Blow up for the critical generalized Korteweg-de Vries equation II: Minimal mass solution, preprint.

[40] Y. MARTEL, F. Merle and T.-P. TSAI, Stability in $H^{1}$ of the sum of $K$ solitary waves for some nonlinear Schrödinger equations, Duke Math. J. 133 (2006), 405-466.

[41] F. MERLE, Construction of solutions with exactly $k$ blow-up points for the Schrödinger equation with critical nonlinearity, Comm. Math. Phys. 129 (1990), 223-240.

[42] F. MERLE, Determination of blow-up solutions with minimal mass for nonlinear Schrödinger equations with critical power, Duke Math. J. 69 (1993), 427-454.

[43] F. MERLE, Nonexistence of minimal blow-up solutions of equations $i u_{t}=-\Delta u-$ $k(x)|u|^{\frac{4}{N}} u$ in $\mathbb{R}^{N}$, Ann. Inst. H. Poincaré Phys. Théor. 64 (1996), 33-85.

[44] F. MERLE, Existence of blow-up solutions in the energy space for the critical generalized KdV equation, J. Amer. Math. Soc. 14 (2001), 555-578.

[45] F. MERLE and P. RAPHAËL, Sharp upper bound on the blow-up rate for the critical nonlinear Schrödinger equation, Geom. Funct. Anal. 13 (2003), 591-642.

[46] F. MERLE and P. RAPHAËL, On universality of blow-up profile for $L^{2}$ critical nonlinear Schrödinger equation, Invent. Math. 156 (2004), 565-672.

[47] F. MERLE and P. RAPHAËL, The blow-up dynamics and upper bound on the blow-up rate for the critical nonlinear Schrödinger equation, Ann. of Math. 161 (2005), 157-222.

[48] F. MERLE and P. RAPHAËL, Profiles and quantization of the blow-up mass for critical nonlinear Schrödinger equation, Comm. Math. Phys. 253 (2005), 675-704.

[49] F. MERLE and P. RAPHAËL, On a sharp lower bound on the blow-up rate for the $L^{2}$ critical nonlinear Schrödinger equation, J. Amer. Math. Soc. 19 (2006), 37-90.

[50] F. Merle, P. RAPHAËL and J. Szeftel, The instability of Bourgain-Wang solutions for the $L^{2}$ critical NLS, Amer. J. Math. 135 (2013), 967-1017.

[51] F. MERLE, P. RAPHAËL and I. RODNIANSKI, Blowup dynamics for smooth data equivariant solutions to the critical Schrödinger map problem, Invent. Math. 193 (2013), 249-365.

[52] K. NAKANISHI and W. SCHLAG, Global dynamics above the ground state energy for the focusing nonlinear Klein-Gordon equation, J. Differential Equations 250 (2011), 22992333. 
[53] K. NAKANISHI and W. SCHLAG, Global dynamics above the ground state energy for the cubic NLS equation in 3D, Calc. Var. Partial Differential Equations 44 (2012), 1-45.

[54] P. RAPHAËL, Stability of the log-log bound for blow-up solutions to the critical non linear Schrödinger equation, Math. Ann. 331 (2005), 577-609.

[55] P. RAPHAËL, "Stability and Blow up for the Nonlinear Schrodinger equation", Lecture notes for the Clay summer school on evolution equations, ETH, Zurich, 2008.

[56] P. RAPHAËL and I. RODNIANSKI, Stable blow-up dynamics for the critical co-rotational Wave Maps and equivariant Yang-Mills problems, Publications scientifiques de l'IHES, to appear. arXiv:0911.0692

[57] P. RAPHAËL and R. SCHWEYER, Stable blow-up dynamics for the 1-corotational energy critical harmonic heat flow, Commun. Pure Appl. Math. 66 (2013), 414-480.

[58] P. RAPHAËL and J. SZEFTEL, Existence and uniqueness of minimal blow-up solutions to an inhomogeneous mass critical NLS, J. Amer. Math. Soc. 23 (2011), to appear. Preprint arXiv:1001.1627

[59] M. J. Landman, G. C. Papanicolaou, C. Sulem and P.-L. Sulem, Rate of blowup for solutions of the nonlinear Schrödinger equation at critical dimension, Phys. Rev. A (3) 38 (1988), 3837-3843.

[60] G. Perelman, On the formation of singularities in solutions of the critical nonlinear Schrödinger equation, Ann. Henri Poincaré 2 (2001), 605-673.

[61] I. RODNIANSKI and J. STERBENZ, On the formation of singularities in the critical $O(3)$ $\sigma$-model, Ann. of Math. (2) 172 (2010), 187-242.

[62] R. SCHWEYER, Type II blow-up for the four dimensional energy critical semi linear heat equation, J. Funct. Anal. 263 (2012), 3922-3983.

[63] S. SHAO, The linear profile decomposition for the Airy equation and the existence of maximizers for the Airy Strichartz inequality, Anal. PDE 2 (2009), 83-117.

[64] G. Talenti, Best constant in Sobolev inequality, Ann. Mat. Pura Appl. 110 (1976), 535372.

[65] M.I. WeINSTEIN, Nonlinear Schrödinger equations and sharp interpolation estimates, Comm. Math. Phys. 87 (1983), 567-576.

[66] M. I. WEINSTEIN, Modulational stability of ground states of nonlinear Schrödinger equations, SIAM J. Math. Anal. 16 (1985), 472-491.

[67] M. I. WEINSTEIN, Lyapunov stability of ground states of nonlinear dispersive evolution equations, Commun. Pure Appl. Math. 39 (1986), 51-68.

Université de Versailles St-Quentin and Institut Universitaire de France LMV CNRS UMR8100

yvan.martel@math.polytechnique.fr

Université de Cergy Pontoise

and Institut des Hautes Etudes Scientifiques

AGM CNRS UMR8088

merle@math.u-cergy.fr

Université Paul Sabatier

and Institut Universitaire de France

Institut de Mathématiques de Toulouse

CNRS UMR 5219

pierre.raphael@math.univ-toulouse.fr 